Supporting Information

\title{
Nickel-Catalyzed Enantioselective Hydroboration of Vinylarenes
}

\author{
Hai N. Tran and Levi M. Stanley \\ Department of Chemistry, Iowa State University, Ames, IA 50011, United States \\ Email: 1stanley@iastate.edu
}

\section{Table of Contents}

1. General Experimental Details $\quad$ S-2

2. Materials $\quad$ S-2

3. Reaction Optimizations $\quad$ S-7

4. General Procedure for Ni-Catalyzed Hydroboration of Vinylarenes with $\mathrm{B}_{2} \mathrm{pin}_{2}$ and Determination of $\begin{array}{ll}\text { Enantiomeric Excesses of Products } & \text { S-12 }\end{array}$

5. Scalability S-17

$\begin{array}{ll}\text { 6. Synthetic Application } & \text { S-17 }\end{array}$

7. Kinetic Studies $\quad$ S-19

8. Protodeboronation Test $\quad$ S-21

8. References $\quad$ S-22

9. HPLC Charts of Chiral Products $\quad$ S-24

10. ${ }^{1} \mathrm{H},{ }^{13} \mathrm{C},{ }^{19} \mathrm{~F}$ and ${ }^{11} \mathrm{~B}$ NMR Spectra for Compounds $\quad$ S-52 


\section{General Experimental Details}

Unless otherwise noted, all reactions were conducted under inert atmosphere in a nitrogen-filled glovebox or by standard Schlenk techniques. All glassware used for reactions were dried at $140{ }^{\circ} \mathrm{C}$ in an oven overnight.

Flash column chromatography was performed on SiliFlash ${ }^{\circledR}$ P60 silica gel (40-63 $\mu \mathrm{m}, 60 \AA$ ) using hexanes/ethyl acetate or hexanes/diethyl ether mixtures as eluents. Reaction products were visualized on TLC by UV light or by staining with $p$-anisaldehyde.

HRMS analysis was performed at the Iowa State University Chemical Instrumentation Facility on an Agilent 6540 QTOF spectrometer (ESI). NMR spectra were acquired on Varian MR-400 and Bruker Avance III 600 spectrometers at the Iowa State University Chemical Instrumentation Facility. Chemical shifts are reported in ppm relative to a residual solvent peak $\left(\mathrm{CDCl}_{3}=7.26 \mathrm{ppm}\right.$ for ${ }^{1} \mathrm{H}$ and $77.16 \mathrm{ppm}$ for

$\left.{ }^{13} \mathrm{C}\right)$. Coupling constants are reported in hertz. HPLC analyses were carried out on a Water Alliance HPLC system with an e2695 Separations Module and a 2489 (UV/Vis) dual wavelength detector, or on an Agilent 1260 Infinity II LC system with 1260 Infinity II Diode Array Detector. HPLC columns (from Daicel Chemical Ind., Ltd) include CHIRALPAK ASH (0.46 cm x $25 \mathrm{~cm})$, CHIRALCEL ODH (0.46 cm x 25 $\mathrm{cm})$, CHIRALCEL OJH $(0.46 \mathrm{~cm}$ x $25 \mathrm{~cm})$, and CHIRALPAK IG $(0.46 \mathrm{~cm}$ x $25 \mathrm{~cm})$. Optical rotations were measured on an Atago AP-300 automatic polarimeter.

\section{Materials}

All chemicals obtained from commercial sources were used directly without further purification. Bis(1,5cyclooctadiene)nickel(0) $\left(\mathrm{Ni}(\mathrm{cod})_{2}\right)$ and tetrakis(triphenylphosphine)palladium $(0) \quad\left(\mathrm{Pd}_{(}\left(\mathrm{PPh}_{3}\right)_{4}\right)$ were purchased from Strem Chemicals. Bis(pinacolato)diboron $\left(\mathrm{B}_{2} \mathrm{pin}_{2}\right)$ and potassium trimethylsilanoate (TMSOK) were purchased from AK Scientific. Tetramethylammonium fluoride tetrahydrate $\left(\mathrm{Me}_{4} \mathrm{NF} \cdot 4 \mathrm{H}_{2} \mathrm{O}\right)$, tetrabutylammonium fluoride hydrate $\left(\mathrm{Bu}_{4} \mathrm{NF}_{2} \mathrm{O}\right)$, tetrabutylammonium fluoride solution $\left(\mathrm{Bu}_{4} \mathrm{NF}, 1 \mathrm{M}\right.$ in THF), cesium fluoride, lithium tert-butoxide ( $\left.t \mathrm{BuOLi}\right)$, sodium methoxide (NaOMe), potassium carbonate $\left(\mathrm{K}_{2} \mathrm{CO}_{3}\right)$, furan, n-butyllithium solution (2.5 $\mathrm{M}$ in hexane), and 4-iodoanisole were purchased form Sigma-Aldrich. Potassium acetate (KOAc), sodium hydroxide $(\mathrm{NaOH})$, and hydrogen peroxide solution $\left(\mathrm{H}_{2} \mathrm{O}_{2} 30 \%\right.$ (w/w) in water) were purchased from Fisher. N-bromosuccinimide (NBS) and silver(I) oxide $\left(\mathrm{Ag}_{2} \mathrm{O}\right)$ were purchased from Acros.

Anhydrous alcohol, anhydrous benzotrifluoride, anhydrous 2-methyltetrahydrofuran (2-MeTHF), anhydrous dimethoxyethane (DME) and anhydrous $m$-xylene were purchased from Sigma-Aldrich. Anhydrous dimethylacetamide (DMAc) and anhydrous dichloroethane (DCE) were purchased from Acros. Benzene, toluene and tetrahydrofuran were purchased from Fisher, degassed by purging with argon for 45 
minutes and dried with a solvent purification system by passing through a one-meter column of activated alumina. Methanol-d $\mathrm{d}_{1}$ (MeOD) was purchased from Sigma-Aldrich and degassed by purging with nitrogen for 45 minutes.

Styrene 1a and 4-fluorostyrene 1d were purchased from Sigma-Aldrich. 4-Methoxystyrene 1b, 4methylstyrene 1c and 2-methylstyrene 1h were purchased from Alfa Aesar. 3-Trifluoromethylstyrene 1g was purchased from Acros. Other vinylarenes and 1-phenyl-1,3-butadiene are known and were synthesized by Wittig reactions according to a reported literature procedure. ${ }^{1}$ Spectral data for 4trifluoromethylstyrene $\mathbf{1 e},{ }^{2} 3$-methoxystyrene $\mathbf{1 f}{ }^{3}$ 3,4-dimethoxystyrene $\quad \mathbf{1 i}^{4}{ }^{4}$ 3,4,5-trimethoxystyrene $\mathbf{1 j}{ }^{5}$ and 1-phenyl-1,3-butadiene $\mathbf{1} \mathbf{k}^{6}$ were in accordance with those reported in the literature.

Ligands $\mathbf{L 1},{ }^{7} \mathbf{L 2},{ }^{8} \mathbf{L 3},{ }^{9}$ and $\mathbf{L 4}^{10}$ are known and were synthesized according to reported literature procedures. Ligand $\mathbf{L 5}$ and triphenylphosphine were purchased from Sigma-Aldrich.

Anhydrous $\mathrm{Me}_{4} \mathrm{NF}$ was obtained by azeotropically removing water of $\mathrm{Me}_{4} \mathrm{NF}_{4} 4 \mathrm{H}_{2} \mathrm{O}$ with benzene using the reported procedure by Clark and co-worker: ${ }^{11}$ Under air, an oven dried, pre-weighed $100-\mathrm{mL}$ round-bottom flask was charged with $\mathrm{Me}_{4} \mathrm{NF}_{4} 4 \mathrm{H}_{2} \mathrm{O}$ (5 g, clear crystals) and benzene (50 mL). A Dean-Stark trap and a condenser were connected, and the solution was refluxed in an oil bath for $12 \mathrm{~h}$. Upon completion, the remaining solvent was removed under reduced pressure (rotary evaporator equipped with a water bath at $\left.50{ }^{\circ} \mathrm{C}\right)$ until the mass of the round-bottom flask remained unchanged to afford anhydrous $\mathrm{Me}_{4} \mathrm{NF}(2.7 \mathrm{~g}$, $96 \%$ yield) as white powder. The anhydrous $\mathrm{Me}_{4} \mathrm{NF}$ was quickly transferred to an oven-dried 20-mL scintillation vial and stored in the freezer of the glovebox.

Racemic 1-phenylethanol was purchased from Acros. Racemic 1-(4-methylphenyl)ethanol was purchased from Sigma-Aldrich. Other racemic alcohols are known and were prepared according to a reported literature procedure. ${ }^{12}$ Spectral data for 1-(4-methoxyphenyl)ethanol, ${ }^{13} 1$-(4-fluorophenyl)ethanol, ${ }^{13} 1$ (4-trifluoromethylphenyl)ethanol, ${ }^{13}$ 1-(3-methoxyphenyl)ethanol, ${ }^{14}$ 1-(3-trifluoromethylphenyl)ethanol, ${ }^{15}$ 1-(2-methylphenyl)ethanol, ${ }^{16}$ 1-(3,4-trimethoxyphenyl)ethanol, ${ }^{17}$ 1-(3,4,5-trimethoxyphenyl)ethanol, ${ }^{17}$ 4-phenylbut-3-en-2-ol ${ }^{14}$ were in accordance with those reported in the literature. 


\subsection{General Procedure for Synthesis of Vinylarenes and 1-Phenyl-1,3-Butadiene}<smiles>[O]c1ccc(C=O)cc1</smiles><smiles>O=C/C=C/c1ccccc1</smiles>

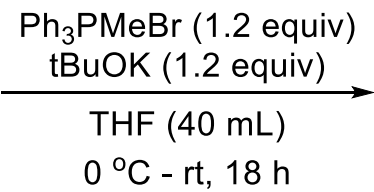<smiles>C=Cc1ccc([O-])cc1</smiles><smiles>C=CC=Cc1ccccc1</smiles>

1 equiv

(10 mmol)

Prepared according to a reported literature procedure by Meggers and co-workers: ${ }^{1}$ An oven-dried, $100-\mathrm{mL}$ round-bottom flask was charged with methyltriphenylphosphonium bromide (12 mmol, 1.2 equiv) and THF $(30 \mathrm{~mL})$. The suspension was cooled to $0{ }^{\circ} \mathrm{C}$ in an ice-water bath. $t \mathrm{BuOK}$ (12 mmol, 1.2 equiv) was then added and the resulting yellow suspension was stirred at $0{ }^{\circ} \mathrm{C}$ for $1 \mathrm{~h}$. To this suspension, a solution of an aldehyde (10 mmol, 1 equiv) in THF $(10 \mathrm{~mL})$ was added dropwise, and the resulting mixture was warmed to room temperature and stirred at room temperature for $18 \mathrm{~h}$. Upon completion, the reaction mixture was filtered over celite. The filtrate was concentrated under reduced pressure and purified by column chromatography to afford the desired product.

\subsection{Ligand Syntheses}

- Ligand L1:

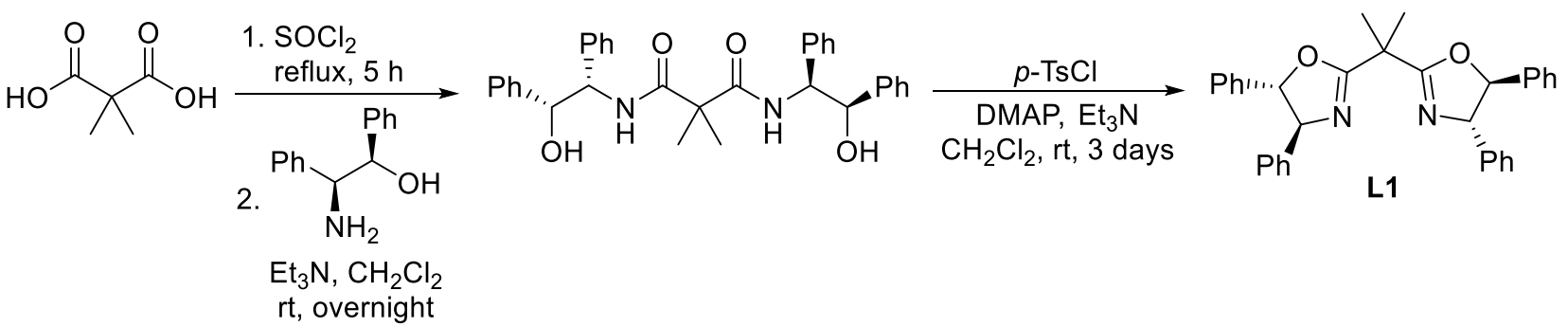

Prepared according to a reported literature procedure by Stanley and co-workers: ${ }^{7}$ A mixture of dimethylmalonic acid $(5 \mathrm{mmol})$ in $\mathrm{SOCl}_{2}(20 \mathrm{~mL})$ was refluxed in an oil bath for 5 hours under air. After cooling to room temperature, the solvent was removed under reduced pressure to afford the crude dimethylmalonyl dichloride, which was directly used for the next step without further purification.

In an oven-dried, 100 -mL round-bottom flask, $(1 R, 2 S)$-2-amino-1,2-diphenylethanol (10 mmol, 2 equiv) was dissolved in DCM (20 mL). The solution was cooled to $0{ }^{\circ} \mathrm{C}$ using an ice-water bath, and triethylamine ( 25 mmol, 5 equiv) was added to the mixture. Next, a solution of crude dimethylmalonyl dichloride in DCM $(10 \mathrm{~mL})$ was added dropwise to the flask. The ice-water bath was removed, and the resulting mixture was 
stirred overnight at room temperature. The reaction mixture was washed with $1 \mathrm{M} \mathrm{HCl}$ and then saturated aqueous $\mathrm{NaHCO}_{3}$. The organic layer was dried over $\mathrm{Na}_{2} \mathrm{SO}_{4}$ and concentrated in vacuo. The resultant bisamide residue was directly used for the final step without further purification.

To an oven-dried, 100 -mL round-bottom flask containing a solution of the bis-amide (1 mmol) in DCM $(20 \mathrm{~mL})$ was added 4-(dimethylamino)pyridine (DMAP, $5 \mathrm{mmol}, 5$ equiv) and triethylamine (5 mmol, 5 equiv). Then, a solution of $p$-toluenesulfonyl chloride (2.5 mmol, 2.5 equiv) in DCM (12 mL) was added at room temperature. The resulting mixture was stirred at room temperature for three days. Upon completion of the reaction, the solution was washed with $\mathrm{NH}_{4} \mathrm{Cl}$ and then $\mathrm{NaHCO}_{3}$. The resulting organic layer was concentrated under reduced pressure and purified by silica gel column chromatography (Hexane/EtOAc $=$ $8 / 2$ ) to afford ligand $\mathbf{L 1}(0.40 \mathrm{~g}, 82 \%$ yield $)$ as a white solid. Spectra data were in accordance with those reported in the literature. ${ }^{7}$

- $\quad$ Ligand L2:<smiles>N#CC1(C#N)CCCCC1</smiles>

$6.5 \mathrm{mmol}$ (1 equiv)<smiles>N[C@@H](CO)c1ccccc1</smiles>

(2.4 equiv)

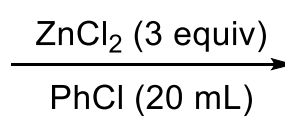

$150{ }^{\circ} \mathrm{C}, 12 \mathrm{~h}$

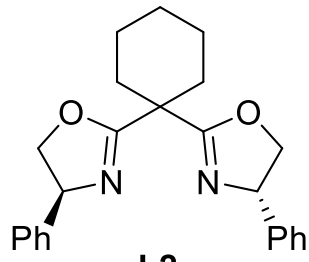

L2

Prepared according to a reported literature procedure by Zhu and co-workers: ${ }^{8}$ An oven-dried, 100-mL round-bottom flask was charged with cyclohexane-1,1-dicarbonitrile (0.872 g, $6.5 \mathrm{mmol}, 1$ equiv), chlorobenzene (20 mL), zinc chloride (2.65 g, $19.5 \mathrm{mmol}, 3$ equiv) and (S)-2-amino-2-phenylethan-1-ol ( $2.14 \mathrm{~g}, 15.6 \mathrm{mmol}, 2.4$ equiv). The reaction mixture was stirred at $150{ }^{\circ} \mathrm{C}$ in an oil bath for $12 \mathrm{~h}$. Upon completion, the reaction mixture was cooled to room temperature. Next, water $(2.5 \mathrm{~mL})$ and ethylenediamine $(5 \mathrm{~mL})$ were added, and the reaction mixture was stirred at room temperature for $1 \mathrm{~h}$. Water $(10 \mathrm{~mL})$ and dichloromethane $(25 \mathrm{~mL})$ were then added to the reaction mixture and the aqueous layer was extracted with dichloromethane $(3 \times 15 \mathrm{~mL})$. The combined organic layers were washed with brine, dried over $\mathrm{MgSO}_{4}$, and the solvent was removed under reduced pressure. The residue was purified by silica gel chromatography (Hexane/EtOAc $=8 / 2)$ to afford ligand $\mathbf{L 2}(0.9 \mathrm{~g}, 38 \%$ yield $)$ as a white solid. Spectra data were in accordance with those reported in the literature. ${ }^{8}$ 


\section{- $\quad$ Ligand L3:}

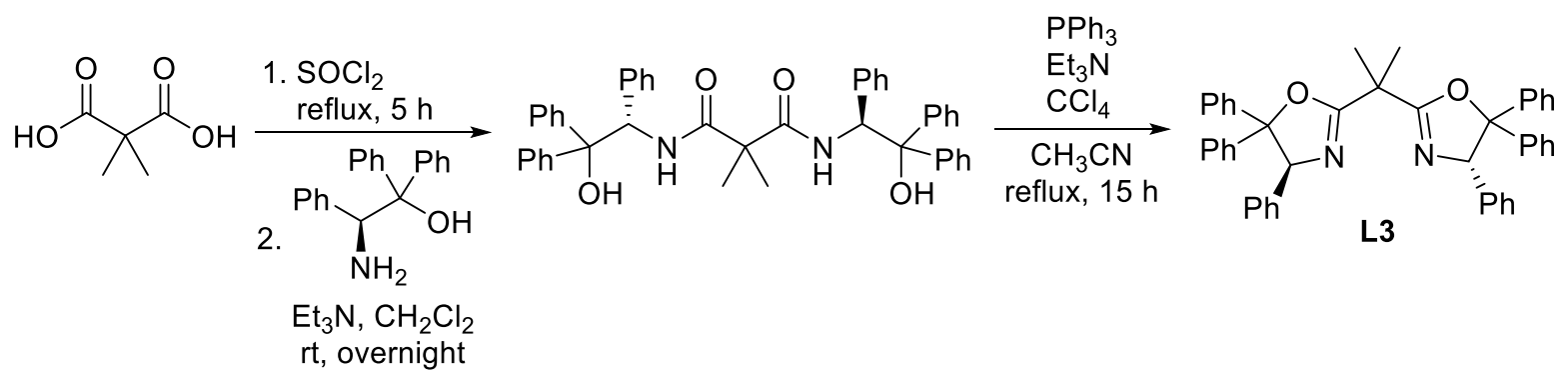

The bis-amide was synthesized using an analogous procedure to that for the synthesis of the corresponding bis-amide of ligand $\mathbf{L} \mathbf{1}$.

Prepared according to a reported literature procedure by You and co-workers: ${ }^{9}$ An oven-dried, 100-mL round-bottom flask was charged with the bis-amide (0.675 g, 1 mmol, 1 equiv) $\mathrm{CH}_{3} \mathrm{CN}$ (20 mL), $\mathrm{PPh}_{3}(1.31$ g, 5 mmol, 5 equiv), $\mathrm{Et}_{3} \mathrm{~N}$ (0.505 g, $5 \mathrm{mmol}, 5$ equiv), and $\mathrm{CCl}_{4}(0.770 \mathrm{~g}, 5 \mathrm{mmol}, 5$ equiv). The reaction mixture was refluxed in an oil bath at $80{ }^{\circ} \mathrm{C}$ for $15 \mathrm{~h}$, then water $(50 \mathrm{~mL})$ was added and the resulting mixture was extracted with $\mathrm{CH}_{2} \mathrm{Cl}_{2}(3 \times 15 \mathrm{~mL})$. The combined organic layers were dried over $\mathrm{Na}_{2} \mathrm{SO}_{4}$, filtered, and the solvent was removed under reduced pressure. The crude product was purified by silica gel column chromatography (Hexane/EtOAc $=10 / 1-5 / 1)$ to give ligand $\mathbf{L 3}(0.45 \mathrm{~g}, 71 \%$ yield $)$ as a white solid. Spectra data were in accordance with those reported in the literature. ${ }^{9}$

- $\quad$ Ligand L4:

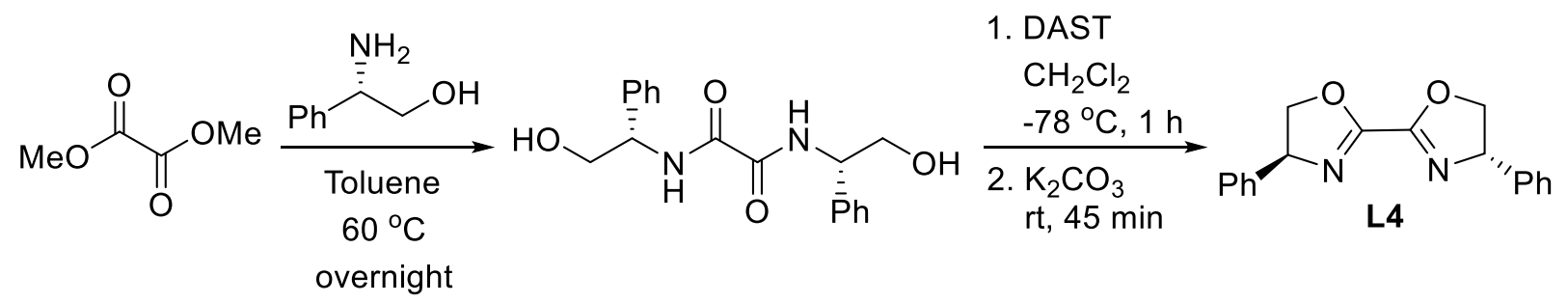

Prepared according to a reported literature procedure by Hu and co-workers: ${ }^{10}$ An oven-dried, 100-mL round-bottom flask was charged with dimethyl oxalate $(0.472 \mathrm{~g}, 4 \mathrm{mmol}, 1.00$ equiv), (S)-2-amino-2phenylethan-1-ol (1.1 g, $8 \mathrm{mmol}, 2$ equiv), and anhydrous toluene. The reaction mixture was stirred in an oil bath at $80{ }^{\circ} \mathrm{C}$ overnight. Upon completion, the reaction mixture was cooled to room temperature and concentrated in vacuo to afford the crude diamide, which was directly used in the next step without further purification.

Bis-amide (0.328 g, $1 \mathrm{mmol}, 1$ equiv) and DCM (20 mL) were added to an oven-dried, 100 -mL roundbottom flask. The flask then was cooled to $-78{ }^{\circ} \mathrm{C}$ in a dry ice-acetone bath, and diethylaminosulfur trifluoride (DAST, $0.39 \mathrm{~mL}, 2.8 \mathrm{mmol}, 2.8$ equiv) was added dropwise. The reaction mixture was stirred 
for $1 \mathrm{~h}$, then $\mathrm{K}_{2} \mathrm{CO}_{3}(0.552 \mathrm{~g}, 4 \mathrm{mmol}, 4$ equiv) was added slowly. The flask was removed from the cold bath, allowed to warm to room temperature and stirred for $45 \mathrm{~min}$. Next, the reaction mixture was diluted with DCM $(20 \mathrm{~mL})$ and water $(30 \mathrm{~mL})$, and the layers were separated. The organic layer was washed with aqueous $\mathrm{NaHCO}_{3}(20 \mathrm{~mL})$ and brine $(30 \mathrm{~mL})$, dried over $\mathrm{Na}_{2} \mathrm{SO}_{4}$, and concentrated under reduced pressure. The crude product was purified by by silica gel column chromatography $($ Hexane/EtOAc $=7 / 3)$ to afford the ligand $\mathbf{L 4}$ (0.22 g, $75 \%$ yield) as a white solid. Spectra data were in accordance with those reported in the literature. ${ }^{10}$

\subsection{General Procedure for Making Racemic Alcohols}<smiles>[R]c1ccc(C(=O)CC)cc1</smiles>

(1 equiv)

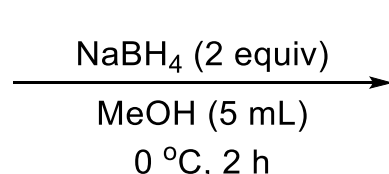

$0{ }^{\circ} \mathrm{C}, 2 \mathrm{~h}$

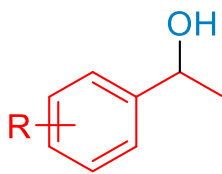

Prepared according to the reported literature procedure by Taylor and co-workers: ${ }^{12}$ Under air, a solution of a respective acetophenone or 4-phenyl-3-buten-2-one $(1.5 \mathrm{mmol})$ in $\mathrm{MeOH}(5 \mathrm{~mL})$ was cooled to $0{ }^{\circ} \mathrm{C}$ in an ice-water bath. $\mathrm{NaBH}_{4}(0.113 \mathrm{~g}, 3 \mathrm{mmol}, 2$ equiv) was then added slowly, and the reaction mixture was stirred at $0{ }^{\circ} \mathrm{C}$ for $2 \mathrm{~h}$. Upon completion, $\mathrm{MeOH}$ was removed under reduced pressure. The resulting mixture was diluted with $\mathrm{CH}_{2} \mathrm{Cl}_{2}(20 \mathrm{~mL})$ and washed with water. The organic layer was dried over anhydrous $\mathrm{Na}_{2} \mathrm{SO}_{4}$, filtered, and concentrated under reduced pressure to afford the benzylic alcohol product. The alcohol was subjected to HPLC analysis without further purification to determine the retention times of the two enantiomers.

\section{Reaction optimization}

Table S1. Base Screening ${ }^{a}$

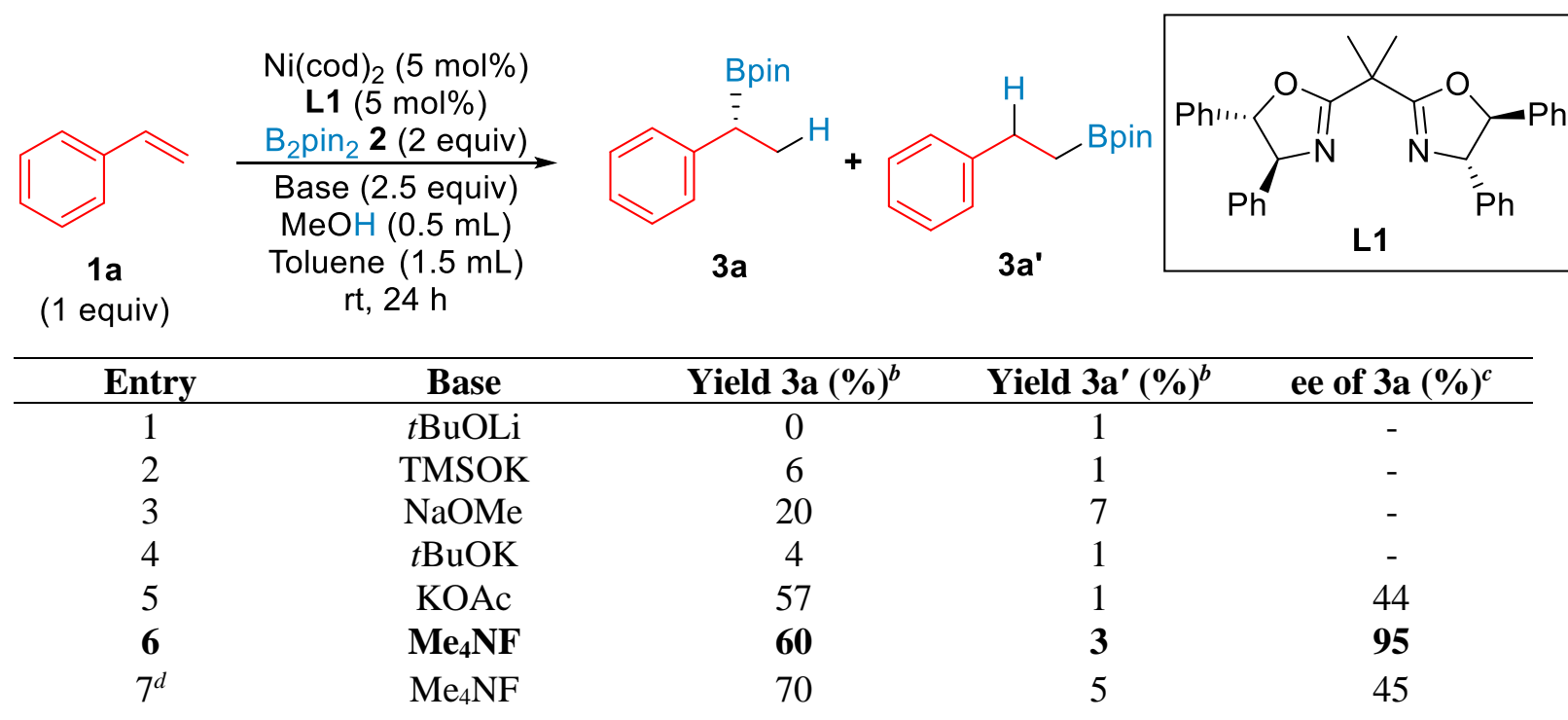




$\begin{array}{ccccc}8 & \mathrm{Me}_{4} \mathrm{NF} .4 \mathrm{H}_{2} \mathrm{O} & 50 & 3 & - \\ 9 & \mathrm{Bu}_{4} \mathrm{NF} . \mathrm{H}_{2} \mathrm{O} & 56 & 3 & 90 \\ 10 & \mathrm{Bu}_{4} \mathrm{NF}(1 \mathrm{M} \text { in THF }) & 45 & 2 & - \\ 11 & \mathrm{CsF} & 47 & 2 & - \\ 12^{e} & \mathrm{Me}_{4} \mathrm{NF} & 66 & 4 & 92 \\ 13^{f} & \mathrm{Me}_{4} \mathrm{NF} & 65 & 4 & 90 \\ 14^{g} & \mathrm{Me}_{4} \mathrm{NF} & 62 & 4 & 89\end{array}$

${ }^{a}$ Reaction condition: 1a $(0.25 \mathrm{mmol}), \mathbf{2}(0.5 \mathrm{mmol}), \mathrm{Ni}(\mathrm{cod}) 2(0.0125 \mathrm{mmol}), \mathbf{L 1}(0.0125 \mathrm{mmol})$, base $(0.625 \mathrm{mmol}), \mathrm{MeOH}$ $(0.5 \mathrm{~mL})$, toluene $(1.5 \mathrm{~mL})$ at room temperature for $24 \mathrm{~h} .{ }^{b}$ Determined by ${ }^{1} \mathrm{H}$ NMR spectroscopy of the crude reaction mixture using dibromomethane as an internal standard. ${ }^{c}$ Determined by chiral HPLC analysis. ${ }^{d} 5$ mol\% $\mathrm{NiBr}_{2}$.glyme used as $\mathrm{Ni}$ (II) precatalyst with $20 \mathrm{~mol} \% \mathrm{Zn}$ powder as reductant. ${ }^{e} 1$ equiv $\mathrm{Me}_{4} \mathrm{NF}(0.25 \mathrm{mmol}) .{ }^{f} 0.5$ equiv $\mathrm{Me}_{4} \mathrm{NF}(0.125 \mathrm{mmol}) .{ }^{g} 0.25 \mathrm{equiv}$ $\mathrm{Me}_{4} \mathrm{NF}(0.0625 \mathrm{mmol})$.

\section{Table S2. Ligand Screening ${ }^{a}$}

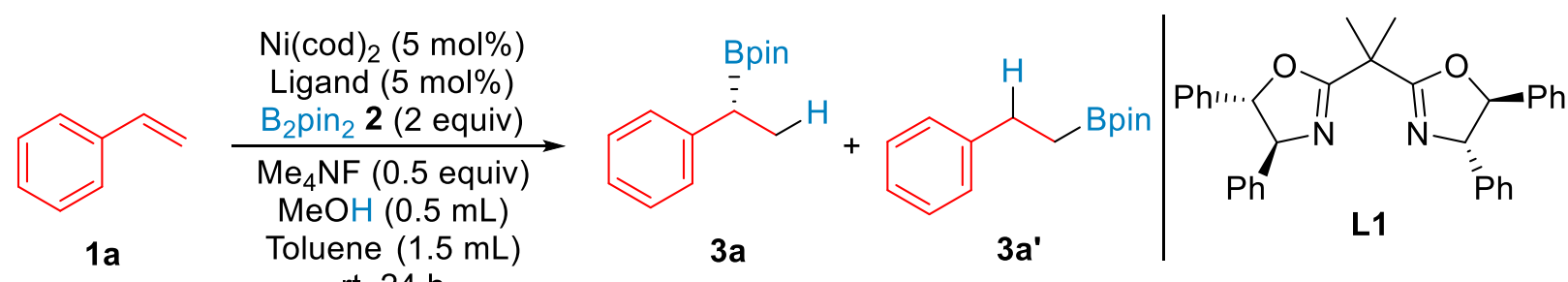<smiles>c1ccc(C2COC(C3(C4=N[C@@H](c5ccccc5)CO4)CCCCC3)=N2)cc1</smiles>

$\mathrm{rt}, 24 \mathrm{~h}$<smiles>CC(C)(C1=NC(c2ccccc2)C(c2ccccc2)(c2ccccc2)O1)C1=NC(c2ccccc2)C(c2ccccc2)(c2ccccc2)O1</smiles>

L3<smiles>c1ccc([C@H]2COC(C3=N[C@H](c4ccccc4)CO3)=N2)cc1</smiles>

L4<smiles>CC(C)C1COC(c2cccc(C3=NC(C(C)C)CO3)n2)=N1</smiles>

L5

\begin{tabular}{|c|c|c|c|c|}
\hline Entry & Ligand & Yield 3a $(\%)^{b}$ & Yield 3a' $(\%)^{b}$ & ee of $3 a(\%)^{c}$ \\
\hline 1 & L1 & 60 & 5 & 90 \\
\hline 2 & L2 & 64 & 2 & 96 \\
\hline 3 & L3 & 71 & 5 & 88 \\
\hline 4 & L4 & 23 & 3 & - \\
\hline 5 & L5 & 0 & 0 & - \\
\hline
\end{tabular}

${ }^{a}$ Reaction condition: 1a $(0.25 \mathrm{mmol}), 2(0.5 \mathrm{mmol}), \mathrm{Ni}(\mathrm{cod}){ }_{2}(0.0125 \mathrm{mmol})$, ligand $(0.0125 \mathrm{mmol}), \mathrm{Me}_{4} \mathrm{NF}(0.125 \mathrm{mmol})$, $\mathrm{MeOH}(0.5 \mathrm{~mL})$, toluene $(1.5 \mathrm{~mL})$ at room temperature for $24 \mathrm{~h} .{ }^{b}$ Determined by ${ }^{1} \mathrm{H}$ NMR spectroscopy of the crude reaction mixture using dibromomethane as an internal standard. ${ }^{c}$ Determined by chiral HPLC analysis. 
Table S3. Hydride Screening ${ }^{a}$

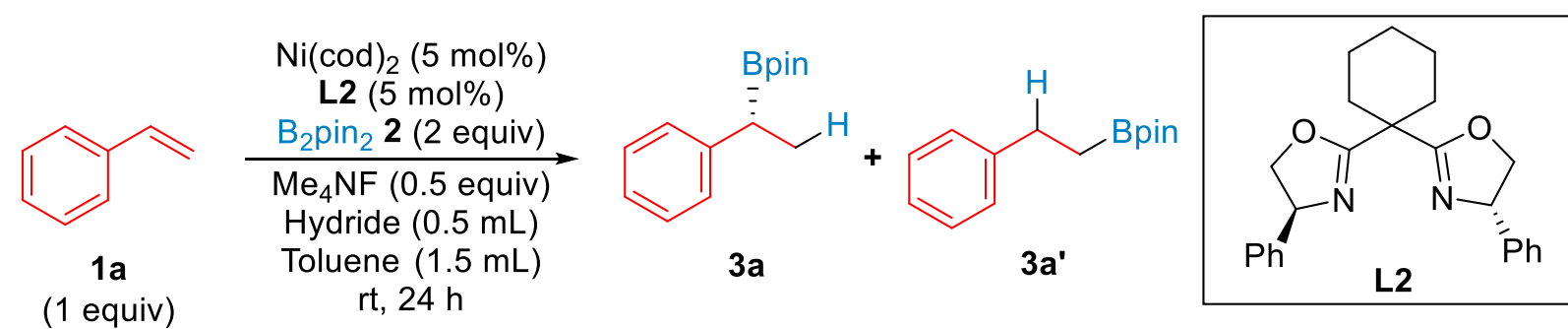

\begin{tabular}{ccccc}
\hline Entry & Hydride & ${\text { Yield 3a }(\%)^{b}}^{b}$ & Yield 3a $^{\mathbf{(}(\%)^{b}}$ & ee of 3a (\%) \\
\hline 1 & $\mathrm{MeOH}$ & 64 & 2 & 96 \\
2 & $\mathrm{nBuOH}$ & 16 & trace & - \\
3 & $i \mathrm{PrOH}$ & 0 & 0 & - \\
4 & $t \mathrm{AmylOH}$ & 0 & 0 & - \\
5 & $\mathrm{H}_{2} \mathrm{O}$ & 0 & 0 & - \\
\hline
\end{tabular}

${ }^{a}$ Reaction condition: 1a $(0.25 \mathrm{mmol}), 2(0.5 \mathrm{mmol}), \mathrm{Ni}(\operatorname{cod})_{2}(0.0125 \mathrm{mmol}), \mathbf{L 2}(0.0125 \mathrm{mmol}), \mathrm{Me}_{4} \mathrm{NF}(0.125 \mathrm{mmol})$, hydride $(0.5 \mathrm{~mL})$, toluene $(1.5 \mathrm{~mL})$ at room temperature for $24 \mathrm{~h} .{ }^{b}$ Determined by ${ }^{1} \mathrm{H}$ NMR spectroscopy of the crude reaction mixture using dibromomethane as an internal standard. ${ }^{c}$ Determined by chiral HPLC analysis.

Table S4. Solvent Screening ${ }^{a}$

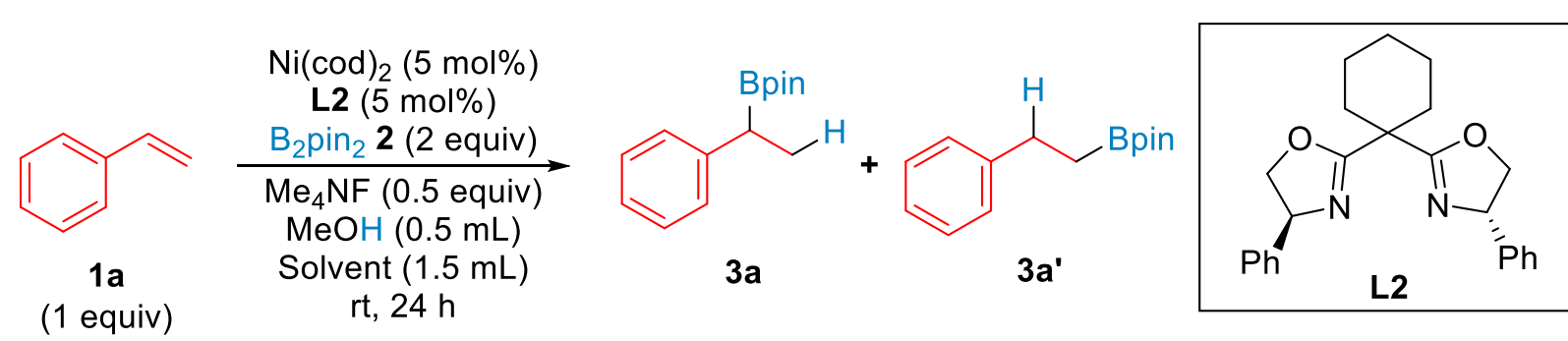

\begin{tabular}{|c|c|c|c|c|}
\hline Entry & Solvent & Yield 3a $(\%)^{b}$ & Yield 3a' $(\%)^{b}$ & ee of $3 a(\%)^{c}$ \\
\hline 1 & Toluene & 64 & 2 & 96 \\
\hline 2 & Benzene & 60 & 3 & 92 \\
\hline 3 & Benzotrifluoride & 48 & 2 & - \\
\hline 4 & $m$-Xylene & 57 & 3 & 90 \\
\hline 5 & DMAc & 0 & 0 & - \\
\hline 6 & 2-MeTHF & 0 & 0 & - \\
\hline 7 & $\mathrm{DCE}$ & 0 & 0 & - \\
\hline
\end{tabular}

${ }^{a}$ Reaction condition: 1a $(0.25 \mathrm{mmol}), 2(0.5 \mathrm{mmol}), \mathrm{Ni}(\mathrm{cod})_{2}(0.0125 \mathrm{mmol}), \mathbf{L 2}(0.0125 \mathrm{mmol}), \mathrm{Me}_{4} \mathrm{NF}(0.125 \mathrm{mmol}), \mathrm{MeOH}$ $(0.5 \mathrm{~mL})$, solvent $(1.5 \mathrm{~mL})$ at room temperature for $24 \mathrm{~h} .{ }^{b}$ Determined by ${ }^{1} \mathrm{H}$ NMR spectroscopy of the crude reaction mixture using dibromomethane as an internal standard. ${ }^{c}$ Determined by chiral HPLC analysis. 
Table S5. Time Study ${ }^{a}$

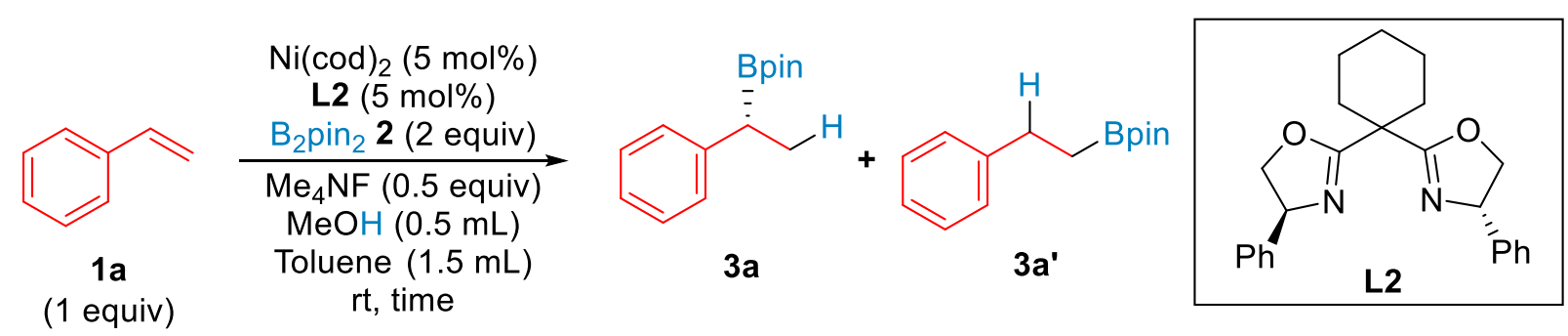

\begin{tabular}{ccccc}
\hline Entry & Time $(\mathbf{h})$ & ${\text { Yield 3a }(\%)^{b}}^{b}$ & Yield 3a' $\left.^{(\%)}\right)^{b}$ & ${\text { ee of 3a }(\%)^{c}}^{\boldsymbol{c}}$ \\
\hline 1 & 1 & 21 & 0 & - \\
2 & 2 & 28 & 0 & - \\
3 & 4 & 40 & 0 & - \\
4 & 8 & 55 & 1 & - \\
5 & 16 & 68 & 0 & 95 \\
6 & 24 & 64 & 2 & 96 \\
\hline
\end{tabular}

${ }^{a}$ Reaction condition: 1a $(0.25 \mathrm{mmol}), 2(0.5 \mathrm{mmol}), \mathrm{Ni}(\mathrm{cod}){ }_{2}(0.0125 \mathrm{mmol}), \mathbf{L 2}(0.0125 \mathrm{mmol}), \mathrm{Me} 4 \mathrm{NF}(0.125 \mathrm{mmol}), \mathrm{MeOH}$ $(0.5 \mathrm{~mL})$, toluene $(1.5 \mathrm{~mL})$ at room temperature for $1-24 \mathrm{~h} .{ }^{b}$ Determined by ${ }^{1} \mathrm{H}$ NMR spectroscopy of the crude reaction mixture using dibromomethane as an internal standard. ${ }^{c}$ Determined by chiral HPLC analysis.

Table S6. Temperature Study ${ }^{a}$

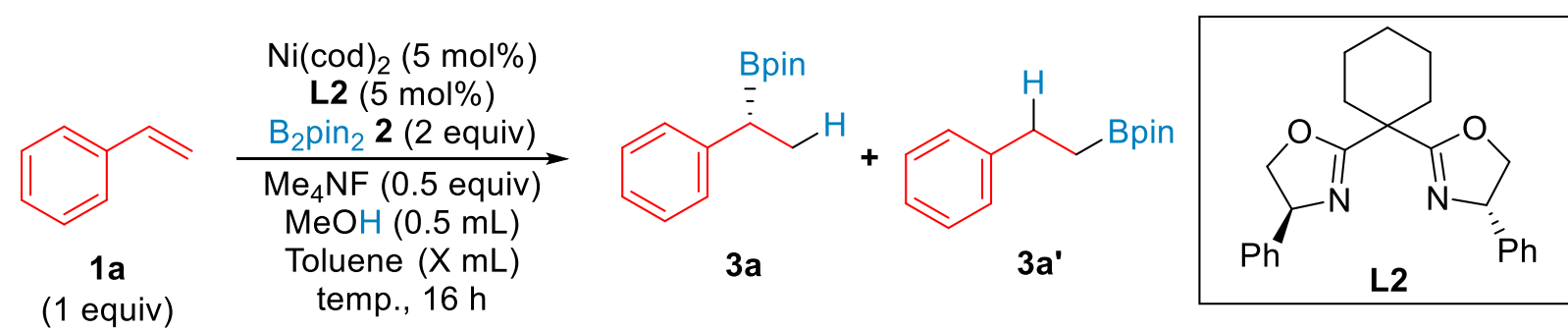

\begin{tabular}{|c|c|c|c|c|}
\hline Entry & Temp. $\left({ }^{\circ} \mathrm{C}\right)$ & Yield 3a $(\%)^{b}$ & Yield 3a' $(\%)^{b}$ & ee of 3a $(\%)^{c}$ \\
\hline 1 & 0 & 36 & 0 & - \\
\hline 2 & 10 & 66 & 0 & 83 \\
\hline 3 & $\mathrm{rt}$ & 68 & 0 & 95 \\
\hline 4 & 40 & 75 & 0 & 92 \\
\hline 5 & 60 & 52 & 0 & - \\
\hline
\end{tabular}

${ }^{a}$ Reaction condition: 1a $(0.25 \mathrm{mmol}), 2(0.5 \mathrm{mmol}), \mathrm{Ni}(\mathrm{cod})_{2}(0.0125 \mathrm{mmol}), \mathbf{L 2}(0.0125 \mathrm{mmol}), \mathrm{Me} 4 \mathrm{NF}(0.125 \mathrm{mmol}), \mathrm{MeOH}$ $(0.5 \mathrm{~mL})$, toluene $(1.5 \mathrm{~mL})$ at $0-60{ }^{\circ} \mathrm{C}$ for $16 \mathrm{~h} .{ }^{b}$ Determined by ${ }^{1} \mathrm{H}$ NMR spectroscopy of the crude reaction mixture using dibromomethane as an internal standard. ${ }^{c}$ Determined by chiral HPLC analysis. 
Table S7. Ni(cod) $)_{2} /$ Ligand (1:1) Loading ${ }^{a}$

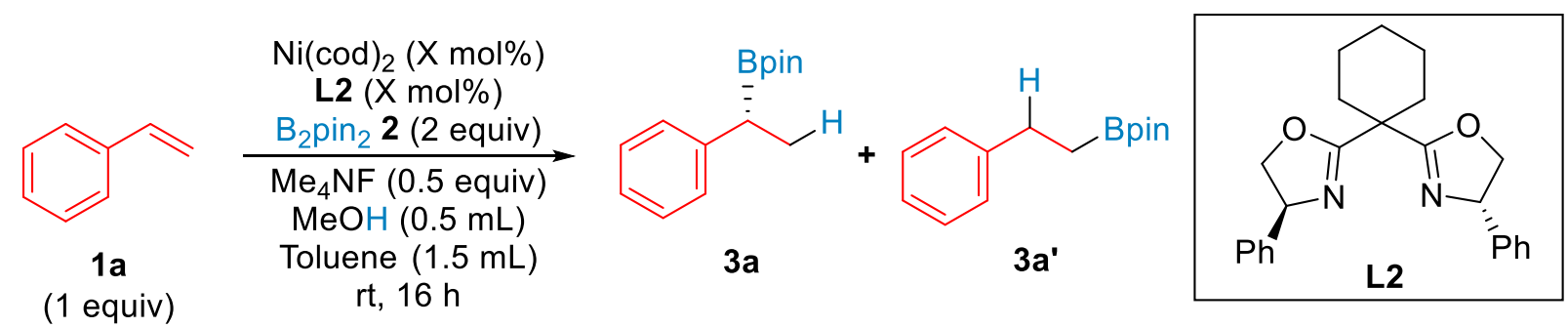

\begin{tabular}{|c|c|c|c|c|}
\hline Entry & $\mathbf{X}$ & Yield 3a $(\%)^{b}$ & Yield 3a' $(\%)^{b}$ & ee of $3 a(\%)^{c}$ \\
\hline 1 & 10 & 48 & 0 & - \\
\hline 2 & 7.5 & 58 & 0 & 92 \\
\hline 3 & 5 & 68 & 0 & 95 \\
\hline 4 & 2.5 & 63 & 0 & 94 \\
\hline 5 & 1 & 40 & 0 & - \\
\hline
\end{tabular}

${ }^{a}$ Reaction condition: 1a $(0.25 \mathrm{mmol}), 2(0.5 \mathrm{mmol}), \mathrm{Ni}(\mathrm{cod})_{2}(0.0025-0.025 \mathrm{mmol}), \mathbf{L 2}(0.0025-0.025 \mathrm{mmol}), \mathrm{Me} 4 \mathrm{NF}(0.125$ $\mathrm{mmol}), \mathrm{MeOH}(0.5 \mathrm{~mL})$, toluene $(1.5 \mathrm{~mL})$ at room temperature for $16 \mathrm{~h} .{ }^{b}$ Determined by ${ }^{1} \mathrm{H}$ NMR spectroscopy of the crude reaction mixture using dibromomethane as an internal standard. ${ }^{c}$ Determined by chiral HPLC analysis.

\section{Table S8. Reaction Concentration ${ }^{a}$}

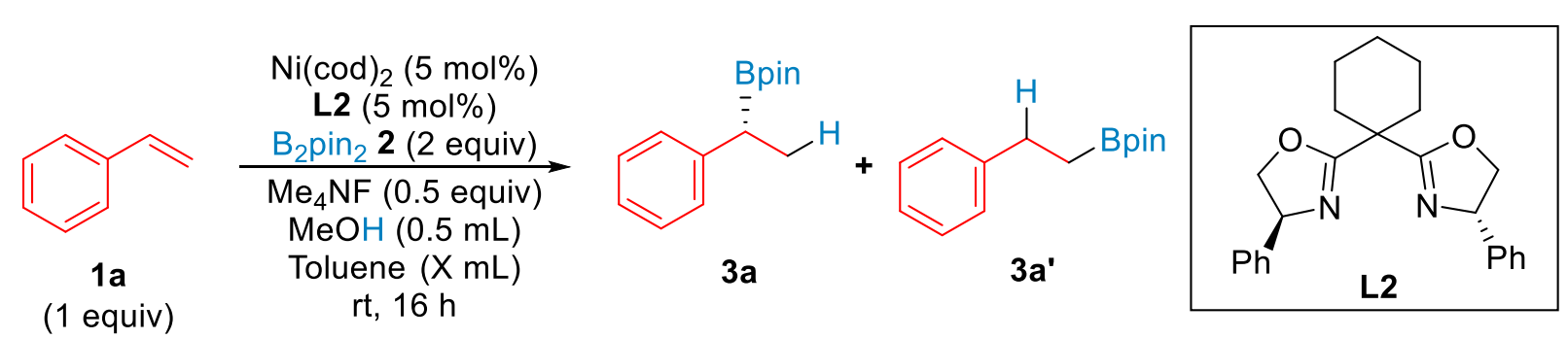

\begin{tabular}{ccccc}
\hline Entry & $\mathbf{X}(\mathbf{m L})$ & ${\text { Yield 3a }(\boldsymbol{\%})^{\boldsymbol{b}}}$ & Yield 3a' (\%) $^{\boldsymbol{b}}$ & ee of 3a $(\boldsymbol{\%})^{\boldsymbol{c}}$ \\
\hline 1 & 0.5 & 86 & 0 & 87 \\
2 & 1 & 84 & 0 & 88 \\
3 & 1.5 & 68 & 0 & 95 \\
4 & 2 & 77 & 0 & 92 \\
5 & 2.5 & 85 & 0 & 94 \\
6 & 3 & 76 & 0 & 91 \\
7 & 3.5 & 78 & 0 & 91 \\
\hline
\end{tabular}

${ }^{a}$ Reaction condition: 1a $\left.(0.25 \mathrm{mmol}), 2(0.5 \mathrm{mmol}), \mathrm{Ni}(\mathrm{cod})\right)_{2}(0.0125 \mathrm{mmol}), \mathbf{L 2}(0.0125 \mathrm{mmol}), \mathrm{Me} 4 \mathrm{NF}(0.125 \mathrm{mmol}), \mathrm{MeOH}$ $(0.5 \mathrm{~mL})$, toluene $(0.5-3.5 \mathrm{~mL})$ at room temperature for $16 \mathrm{~h} .{ }^{b}$ Determined by ${ }^{1} \mathrm{H}$ NMR spectroscopy of the crude reaction mixture using dibromomethane as an internal standard. ${ }^{c}$ Determined by chiral HPLC analysis. 


\section{General Procedure for Ni-Catalyzed Hydroboration of Vinylarenes with $\mathbf{B}_{2}$ pin $_{2}$ and Determination of Enantiomeric Excesses of Products}

\subsection{General Procedure for Ni-Catalyzed Hydroboration of Vinylarenes with $B_{2}$ pin $_{2}$}

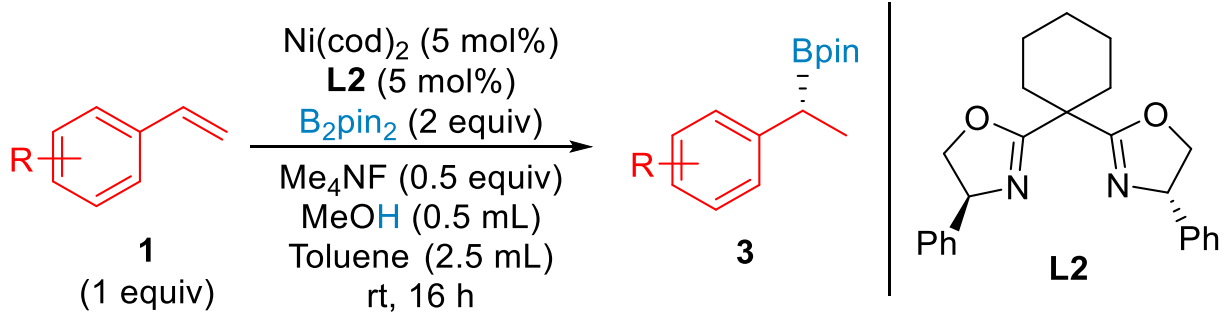

In a nitrogen-filled glovebox, an oven dried 1-dram vial was charged with $\mathrm{Ni}(\mathrm{cod})_{2}(3.5 \mathrm{mg}, 0.0125 \mathrm{mmol})$, $\mathbf{L 2}$ (4.7 mg, $0.0125 \mathrm{mmol}$ ), anhydrous $\mathrm{Me}_{4} \mathrm{NF}$ (11.6 mg, $0.125 \mathrm{mmol}$ ), and $\mathrm{B}_{2} \operatorname{pin}_{2} 2$ (127 mg, $\left.0.5 \mathrm{mmol}\right)$. Methanol $(0.5 \mathrm{~mL})$ and toluene $(0.5,1.5$ or $2.5 \mathrm{~mL})$ were then added, and the resulting mixture was gently stirred at room temperature until clear. Next, the appropriate vinylarene $\mathbf{1 a - 1 j}$ or 1-phenyl-1,3-butadiene 1k $(0.25 \mathrm{mmol})$ was added and the vial was sealed and taken out of the glovebox. The reaction mixture was stirred at room temperature for 16 hours. Upon completion, the reaction mixture was filtered through a short plug of silica gel eluting with DCM and concentrated under reduced pressure. The crude product was purified by flash column chromatography to give the hydroboration products $\mathbf{3 a - 3 k}$. The absolute configuration of the chiral product 3a was assigned by comparison to the sign of optical rotation reported in the literature. ${ }^{18}$ Absolute configurations of additional chiral boronate esters $\mathbf{3 b - 3} \mathbf{k}$ were assigned based on the sense of absolute configuration observed for compound $\mathbf{3 a}$.

\subsection{General Procedure for Determination of Enantiomeric Excesses of Products}

Following the reported literature procedure by $\mathrm{Fu}$ and co-workers: ${ }^{19}$ In a 1-dram vial, the purified product $3(\sim 15 \mathrm{mg})$ was stereospecifically oxidized with $\mathrm{NaBO}_{3} \cdot 4 \mathrm{H}_{2} \mathrm{O}(\sim 100 \mathrm{mg})$ in $\mathrm{THF} / \mathrm{H}_{2} \mathrm{O}(1: 1 ; 2.0 \mathrm{~mL})$ at room temperature for $4 \mathrm{~h}$ under air. Upon completion, the reaction mixture was extracted with $\mathrm{Et}_{2} \mathrm{O}$ (4 x 2 $\mathrm{mL}$ ). The organic layers were combined, dried over anhydrous $\mathrm{Na}_{2} \mathrm{SO}_{4}$, and the solvents were removed under reduced pressure. The resulting benzylic alcohol was subjected to HPLC analysis without further purification to determine the enantiomeric excess.

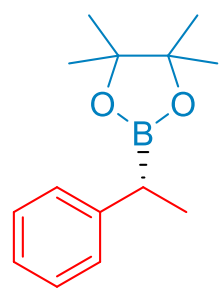

$3 \mathbf{a}$

(R)-2-(1-phenylethyl)-4,4,5,5-tetramethyl-1,3,2-dioxaborolane (3a): Prepared according to the general procedure from styrene $1 \mathbf{a}(26.0 \mathrm{mg}, 0.25 \mathrm{mmol})$ and $\mathrm{B}_{2} \operatorname{pin}_{2} 2(127.0 \mathrm{mg}, 0.5$ mmol) using $2.5 \mathrm{~mL}$ toluene. The crude reaction was purified by flash column chromatography using hexanes/EtOAc $=30: 1$ as the eluent to afford 3a as a colorless oil in $80 \%$ yield $(46.4 \mathrm{mg}, 0.200 \mathrm{mmol})$. Optical rotation: $[\alpha]_{\mathrm{D}}{ }^{24}=-1.49^{\circ}\left(\mathrm{c} 0.74, \mathrm{CHCl}_{3}\right)$. The enantiomeric excess ( $94 \%$ ee) was determined after stereospecifically oxidized to the corresponding alcohol by HPLC (CHIRALCEL ODH, $i \mathrm{PrOH} / \mathrm{hexane}=5 / 95$, flow rate $=1.0 \mathrm{~mL} / \mathrm{min}, \mathrm{t}=$ 
$\left.25{ }^{\circ} \mathrm{C}, \lambda=220 \mathrm{~nm}\right) \mathrm{t}_{\mathrm{R}}=8.4 \mathrm{~min}$ (major), $9.1 \mathrm{~min}$ (minor). ${ }^{1} \mathbf{H} \mathbf{~ N M R}\left(400 \mathrm{MHz}, \mathrm{CDCl}_{3}\right) \delta 7.28-7.20(\mathrm{~m}$, 4H), 7.14-7.10 (m, 1H), 2.43 (q, $J=7.2 \mathrm{~Hz}, 1 \mathrm{H}), 1.32$ (d, $J=7.6 \mathrm{~Hz}, 3 \mathrm{H}), 1.20(\mathrm{~s}, 6 \mathrm{H}), 1.19$ (s, 6H). ${ }^{13} \mathrm{C}$ NMR $\left(101 \mathrm{MHz}, \mathrm{CDCl}_{3}\right) \delta 150.0,128.3,127.8,125.1,83.3,24.6,24.5,17.1$. The NMR spectra were in accordance with those reported in the literature. ${ }^{18}$

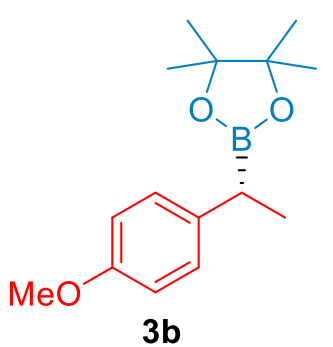

3b

(R)-2-(1-(4-methoxyphenyl)ethyl)-4,4,5,5-tetramethyl-1,3,2-dioxaborolane (3b): Prepared according to the general procedure from 4-methoxystyrene $\mathbf{1 b}(33.5 \mathrm{mg}$, $0.25 \mathrm{mmol})$ and $\mathrm{B}_{2} \operatorname{pin}_{2} 2(127.0 \mathrm{mg}, 0.5 \mathrm{mmol})$ using $2.5 \mathrm{~mL}$ toluene. The crude reaction was purified by flash column chromatography using hexanes/EtOAc $=30: 1$ as the eluent to afford $\mathbf{3 b}$ as a colorless oil in $75 \%$ yield $(49.2 \mathrm{mg}, 0.188 \mathrm{mmol})$. Optical rotation: $[\alpha]_{\mathrm{D}}^{24}=-1.60^{\circ}\left(\mathrm{c} 0.6, \mathrm{CHCl}_{3}\right)$. The enantiomeric excess $(91 \%$ ee $)$ was determined after stereospecifically oxidized to the corresponding alcohol by HPLC (CHIRALPAK ASH, $i \mathrm{PrOH} /$ hexane $=5 / 95$, flow rate $\left.=1.0 \mathrm{~mL} / \mathrm{min}, \mathrm{t}=25^{\circ} \mathrm{C}, \lambda=220 \mathrm{~nm}\right) \mathrm{t}_{\mathrm{R}}=11.0 \mathrm{~min}($ minor $), 12.4$ $\min$ (major). ${ }^{1} \mathbf{H}$ NMR $\left(400 \mathrm{MHz}, \mathrm{CDCl}_{3}\right) \delta 7.13(\mathrm{~d}, J=8.4 \mathrm{~Hz}, 2 \mathrm{H}), 6.82(\mathrm{~d}, J=8.4 \mathrm{~Hz}, 2 \mathrm{H}), 3.78(\mathrm{~s}, 3 \mathrm{H})$, $2.37(\mathrm{q}, J=7.6 \mathrm{~Hz}, 1 \mathrm{H}), 1.30(\mathrm{~d}, J=7.6 \mathrm{~Hz}, 3 \mathrm{H}), 1.21(\mathrm{~s}, 6 \mathrm{H}), 1.20(\mathrm{~s}, 6 \mathrm{H}) .{ }^{13} \mathbf{C ~ N M R}\left(101 \mathrm{MHz}, \mathrm{CDCl}_{3}\right)$ $\delta 157.2,137.0,128.6,113.8,83.3,55.2,24.6,24.5,17.4$. The NMR spectra were in accordance with those reported in the literature. ${ }^{20}$

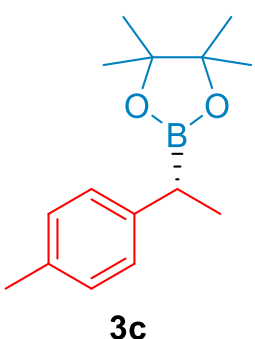

(R)-2-(1-(4-methylphenyl)ethyl)-4,4,5,5-tetramethyl-1,3,2-dioxaborolane

(3c):

Prepared according to the general procedure from 4-methylstyrene 1c $(29.5 \mathrm{mg}, 0.25$ $\mathrm{mmol}$ ) and $\mathrm{B}_{2} \operatorname{pin}_{2} 2$ (127.0 $\left.\mathrm{mg}, 0.5 \mathrm{mmol}\right)$ using $2.5 \mathrm{~mL}$ toluene. The crude reaction was purified by flash column chromatography using hexanes/EtOAc $=30: 1$ as the eluent to afford $3 \mathrm{c}$ as a colorless oil in $76 \%$ yield $(46.7 \mathrm{mg}, 0.190 \mathrm{mmol})$. Optical rotation: $[\alpha]_{\mathrm{D}}{ }^{24}$ $=-1.30^{\circ}\left(\mathrm{c} 0.74, \mathrm{CHCl}_{3}\right)$. The enantiomeric excess $(90 \%$ ee $)$ was determined after stereospecifically oxidized to the corresponding alcohol by HPLC $(\mathrm{CHIRALPAK}$ IG, $i \mathrm{PrOH} / \mathrm{hexane}=$ 3/97, flow rate $=1.0 \mathrm{~mL} / \mathrm{min}, \mathrm{t}=25^{\circ} \mathrm{C}, \lambda=210 \mathrm{~nm}$ ) $\mathrm{t}_{\mathrm{R}}=12.5 \mathrm{~min}$ (major), $13.8 \mathrm{~min}$ (minor). ${ }^{1} \mathbf{H} \mathbf{N M R}$ $\left(400 \mathrm{MHz}, \mathrm{CDCl}_{3}\right) \delta 7.11(\mathrm{~d}, J=8.0 \mathrm{~Hz}, 2 \mathrm{H}), 7.07(\mathrm{~d}, J=8.0 \mathrm{~Hz}, 2 \mathrm{H}), 2.39(\mathrm{q}, J=7.6 \mathrm{~Hz}, 1 \mathrm{H}), 2.30(\mathrm{~s}$, $3 \mathrm{H}), 1.30(\mathrm{~d}, J=7.6 \mathrm{~Hz}, 3 \mathrm{H}), 1.21(\mathrm{~s}, 6 \mathrm{H}), 1.20(\mathrm{~s}, 6 \mathrm{H}) .{ }^{13} \mathrm{C}$ NMR $\left(101 \mathrm{MHz}, \mathrm{CDCl}_{3}\right) \delta 141.9,134.4$, 129.0, 127.6, 83.2, 24.6, 24.5, 21.0, 17.4. The NMR spectra were in accordance with those reported in the literature. $^{21}$

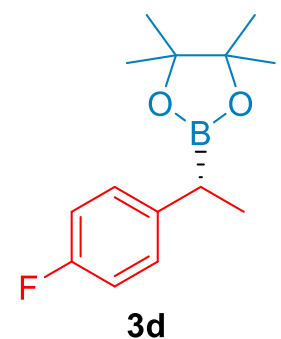

\section{(R)-2-(1-(4-fluorophenyl)ethyl)-4,4,5,5-tetramethyl-1,3,2-dioxaborolane}

Prepared according to the general procedure from 4-fluorostyrene 1d (30.5 mg, 0.25 $\mathrm{mmol}$ ) and $\mathrm{B}_{2} \operatorname{pin}_{2} 2$ (127.0 $\left.\mathrm{mg}, 0.5 \mathrm{mmol}\right)$ using $2.5 \mathrm{~mL}$ toluene. The crude reaction was purified by flash column chromatography using hexanes/EtOAc $=30 / 1$ as the eluent to afford $\mathbf{3 d}$ as a colorless oil in $70 \%$ yield $(43.8 \mathrm{mg}, 0.175 \mathrm{mmol})$. Optical 
rotation: $[\alpha]_{\mathrm{D}}{ }^{24}=-1.59^{\circ}$ (c $0.64, \mathrm{CHCl}_{3}$. The enantiomeric excess $(83 \%$ ee) was determined after stereospecifically oxidized to the corresponding alcohol by HPLC $(\mathrm{CHIRALPAK} \mathrm{IG,} i \mathrm{PrOH} / \mathrm{hexane}=$ 3/97, flow rate $=1.0 \mathrm{~mL} / \mathrm{min}, \mathrm{t}=25^{\circ} \mathrm{C}, \lambda=210 \mathrm{~nm}$ ) $\mathrm{t}_{\mathrm{R}}=11.0 \mathrm{~min}$ (major), $11.7 \mathrm{~min}$ (minor). ${ }^{1} \mathbf{H} \mathbf{~ N M R}$ $\left(400 \mathrm{MHz}, \mathrm{CDCl}_{3}\right) \delta 7.16(\mathrm{dd}, J=8.4,5.6 \mathrm{~Hz}, 2 \mathrm{H}), 6.94(\mathrm{t}, J=8.8 \mathrm{~Hz}, 2 \mathrm{H}), 2.41(\mathrm{q}, J=7.6 \mathrm{~Hz}, 1 \mathrm{H}), 1.30$ $(\mathrm{d}, J=7.6 \mathrm{~Hz}, 3 \mathrm{H}), 1.21(\mathrm{~s}, 6 \mathrm{H}), 1.20(\mathrm{~s}, 6 \mathrm{H}) .{ }^{13} \mathbf{C ~ N M R}\left(101 \mathrm{MHz}, \mathrm{CDCl}_{3}\right) \delta 160.8\left(\mathrm{~d}, J_{\mathrm{C}-\mathrm{F}}=243.4 \mathrm{~Hz}\right)$, $140.5\left(\mathrm{~d}, J_{\mathrm{C}-\mathrm{F}}=3.0 \mathrm{~Hz}\right), 129.0\left(\mathrm{~d}, J_{\mathrm{C}-\mathrm{F}}=8.1 \mathrm{~Hz}\right), 115.0\left(\mathrm{~d}, J_{\mathrm{C}-\mathrm{F}}=21.2 \mathrm{~Hz}\right), 83.4,24.6,24.5,17.2 .{ }^{19} \mathbf{F} \mathbf{~ N M R}$ $\left(376 \mathrm{MHz}, \mathrm{CDCl}_{3}\right) \delta-119.03$. The NMR spectra were in accordance with those reported in the literature. ${ }^{20}$

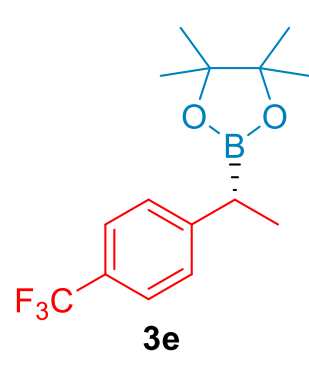

(R)-2-(1-(4-trifluoromethylphenyl)ethyl)-4,4,5,5-tetramethyl-1,3,2-dioxaborolane (3e): Prepared according to the general procedure from 4-trifluoromethylstyrene 1e (48.0 mg, $0.25 \mathrm{mmol})$ and $\mathrm{B}_{2} \operatorname{pin}_{2} 2(127.0 \mathrm{mg}, 0.5 \mathrm{mmol})$ using $1.5 \mathrm{~mL}$ toluene. The crude reaction was purified by flash column chromatography using hexanes/EtOAc $=$ $30: 1$ as the eluent to afford $3 \mathbf{e}$ as a colorless oil in $60 \%$ yield $(48.0 \mathrm{mg}, 0.150 \mathrm{mmol})$. Optical rotation: $[\alpha]_{\mathrm{D}}{ }^{24}=-1.89^{\circ}\left(\mathrm{c} 0.78, \mathrm{CHCl}_{3}\right)$. The enantiomeric excess $(83 \%$ ee) was determined after stereospecifically oxidized to the corresponding alcohol by HPLC (CHIRALPAK IG, $i \mathrm{PrOH} / \mathrm{hexane}=3 / 97$, flow rate $=1.0 \mathrm{~mL} / \mathrm{min}, \mathrm{t}=25^{\circ} \mathrm{C}, \lambda=210 \mathrm{~nm}$ ) $\mathrm{t}_{\mathrm{R}}=7.4 \mathrm{~min}$ (major), $7.8 \mathrm{~min}$ (minor). ${ }^{1} \mathbf{H}$ NMR $\left(400 \mathrm{MHz}, \mathrm{CDCl}_{3}\right) \delta 7.51(\mathrm{~d}, J=8.0,2 \mathrm{H}), 7.31(\mathrm{~d}, J=8.0 \mathrm{~Hz}, 2 \mathrm{H}), 2.49(\mathrm{q}, J=7.2 \mathrm{~Hz}, 1 \mathrm{H}), 1.34$ $(\mathrm{d}, J=7.6 \mathrm{~Hz}, 3 \mathrm{H}), 1.21(\mathrm{~s}, 6 \mathrm{H}), 1.20(\mathrm{~s}, 6 \mathrm{H}) .{ }^{13} \mathrm{C} \mathbf{N M R}\left(101 \mathrm{MHz}, \mathrm{CDCl}_{3}\right) \delta 149.3,128.3,127.7\left(\mathrm{q}, J_{\mathrm{C}-\mathrm{F}}\right.$ $=32.3 \mathrm{~Hz}), 125.2\left(\mathrm{q}, J_{\mathrm{C}-\mathrm{F}}=3.0 \mathrm{~Hz}\right), 124.5\left(\mathrm{q}, J_{\mathrm{C}-\mathrm{F}}=272.7 \mathrm{~Hz}\right), 83.6,24.6,24.5,16.7 .{ }^{19} \mathbf{F ~ N M R}(376 \mathrm{MHz}$, $\left.\mathrm{CDCl}_{3}\right) \delta$-62.2. The NMR spectra were in accordance with those reported in the literature. ${ }^{22}$

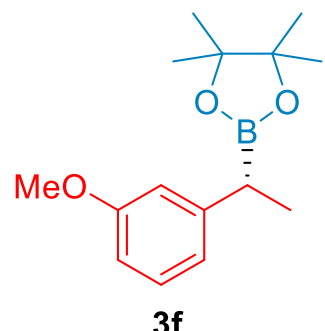

$3 f$

\section{(R)-2-(1-(3-methoxyphenyl)ethyl)-4,4,5,5-tetramethyl-1,3,2-dioxaborolane (3f):}

Prepared according to the general procedure from 3-methoxystyrene 1f $(33.5 \mathrm{mg}$, $0.25 \mathrm{mmol})$ and $\mathrm{B}_{2} \operatorname{pin}_{2} 2(127.0 \mathrm{mg}, 0.5 \mathrm{mmol})$ using $1.5 \mathrm{~mL}$ toluene. The crude reaction was purified by flash column chromatography using hexanes/EtOAc $=30: 1$ as the eluent to afford $\mathbf{3 f}$ as a colorless oil in $60 \%$ yield $(39.3 \mathrm{mg}, 0.150 \mathrm{mmol})$. Optical rotation: $[\alpha]_{\mathrm{D}}{ }^{24}=-1.70^{\circ}\left(\mathrm{c} 0.86, \mathrm{CHCl}_{3}\right)$. The enantiomeric excess $(88 \%$ ee)

was determined after stereospecifically oxidized to the corresponding alcohol by HPLC (CHIRALCEL $\mathrm{ODH}, i \mathrm{PrOH} / \mathrm{h}$ exane $=3 / 97$, flow rate $=1.0 \mathrm{~mL} / \mathrm{min}, \mathrm{t}=25^{\circ} \mathrm{C}, \lambda=220 \mathrm{~nm}$ ) $\mathrm{t}_{\mathrm{R}}=7.4 \mathrm{~min}$ (major), $8.1 \mathrm{~min}$ (minor). ${ }^{1} \mathbf{H}$ NMR $\left(400 \mathrm{MHz}, \mathrm{CDCl}_{3}\right) \delta 7.17(\mathrm{t}, J=8.0 \mathrm{~Hz}, 1 \mathrm{H}), 6.83-6.80(\mathrm{~m}, 1 \mathrm{H}), 6.78(\mathrm{t}, J=2 \mathrm{~Hz}, 1 \mathrm{H})$, 6.69 (ddd, $J=8.2,2.6,0.9 \mathrm{~Hz}, 1 \mathrm{H}), 3.79$ (s, 3H), 2.42 (q, $J=7.2 \mathrm{~Hz}, 1 \mathrm{H}), 1.32$ (d, $J=7.6 \mathrm{~Hz}, 3 \mathrm{H}), 1.22$ (s, $6 \mathrm{H}), 1.21(\mathrm{~s}, 6 \mathrm{H}) .{ }^{13} \mathrm{C}$ NMR $\left(101 \mathrm{MHz}, \mathrm{CDCl}_{3}\right) \delta 159.6,146.6,129.2,120.3,113.4,110.5,83.3,55.1,24.6$, 24.5, 17.0. The NMR spectra were in accordance with those reported in the literature. ${ }^{23}$ 


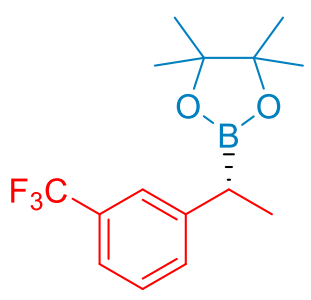

$3 \mathbf{g}$

\section{(R)-2-(1-(3-trifluoromethylphenyl)ethyl)-4,4,5,5-tetramethyl-1,3,2-dioxaborolane}

(3g): Prepared according to the general procedure from 3-trifluoromethylstyrene $\mathbf{1 g}$ (48.0 mg, $0.25 \mathrm{mmol})$ and $\mathrm{B}_{2} \operatorname{pin}_{2} 2(127.0 \mathrm{mg}, 0.5 \mathrm{mmol})$ using $0.5 \mathrm{~mL}$ toluene. The crude reaction was purified by flash column chromatography using hexanes/EtOAc $=$ $30: 1$ as the eluent to afford $\mathbf{3 g}$ as a colorless oil in $50 \%$ yield $(40.0 \mathrm{mg}, 0.125 \mathrm{mmol})$. Optical rotation: $[\alpha]_{\mathrm{D}}{ }^{24}=-1.33^{\circ}\left(\mathrm{c} 0.72, \mathrm{CHCl}_{3}\right)$. The enantiomeric excess $(82 \%$ ee $)$ was determined after stereospecifically oxidized to the corresponding alcohol by HPLC (CHIRALCEL $\mathrm{OJH}, i \mathrm{PrOH} / \mathrm{hexane}=2 / 98$, flow rate $\left.=1.0 \mathrm{~mL} / \mathrm{min}, \mathrm{t}=25^{\circ} \mathrm{C}, \lambda=220 \mathrm{~nm}\right) \mathrm{t}_{\mathrm{R}}=7.1 \mathrm{~min}($ minor $), 7.4 \mathrm{~min}$ (major). ${ }^{1} \mathbf{H}$ NMR (400 MHz, $\left.\mathrm{CDCl}_{3}\right) \delta 7.48(\mathrm{~s}, 1 \mathrm{H}), 7.43-7.34(\mathrm{~m}, 3 \mathrm{H}), 2.50$ (q, J=7.2 Hz, 1H), 1.35 (d, $J=7.6 \mathrm{~Hz}, 3 \mathrm{H}), 1.21(\mathrm{~s}, 6 \mathrm{H}), 1.20(\mathrm{~s}, 6 \mathrm{H}) .{ }^{13} \mathbf{C} \mathbf{N M R}\left(101 \mathrm{MHz}, \mathrm{CDCl}_{3}\right) \delta 145.9,131.2\left(\mathrm{q}, J_{\mathrm{C}-\mathrm{F}}=31.3 \mathrm{~Hz}\right)$, $130.4\left(\mathrm{q}, J_{\mathrm{C}-\mathrm{F}}=31.3 \mathrm{~Hz}\right), 128.6,124.4\left(\mathrm{q}, J_{\mathrm{C}-\mathrm{F}}=273.7 \mathrm{~Hz}\right), 124.5\left(\mathrm{q}, J_{\mathrm{C}-\mathrm{F}}=4.0 \mathrm{~Hz}\right), 122.0\left(\mathrm{q}, J_{\mathrm{C}-\mathrm{F}}=4.0\right.$ $\mathrm{Hz}), 83.5,24.6,24.5,16.8 .{ }^{19} \mathbf{F}$ NMR $\left(376 \mathrm{MHz}, \mathrm{CDCl}_{3}\right) \delta$-62.6. The NMR spectra were in accordance with those reported in the literature. ${ }^{21}$

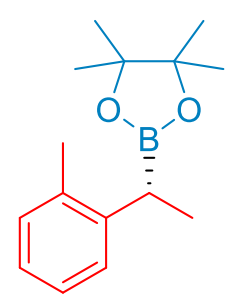

$3 h$

(R)-2-(1-(2-methylphenyl)ethyl)-4,4,5,5-tetramethyl-1,3,2-dioxaborolane (3h): Prepared according to the general procedure from 2-methylstyrene $\mathbf{1 h}(29.5 \mathrm{mg}, 0.25 \mathrm{mmol})$ and $\mathrm{B}_{2} \operatorname{pin}_{2} 2$ (127.0 mg, $\left.0.5 \mathrm{mmol}\right)$ using $2.5 \mathrm{~mL}$ toluene. The crude reaction was purified by flash column chromatography using hexanes/EtOAc $=30: 1$ as the eluent to afford $\mathbf{3 g}$ as a colorless oil in $30 \%$ yield $(18.5 \mathrm{mg}, 0.075 \mathrm{mmol})$. Optical rotation: $[\alpha]_{\mathrm{D}}{ }^{24}=-1.80^{\circ}(\mathrm{c} 0.62$, $\mathrm{CHCl}_{3}$ ). The enantiomeric excess (89\% ee) was determined after stereospecifically oxidized to the corresponding alcohol by HPLC (CHIRALPAK IG, $i \mathrm{PrOH} /$ hexane $=3 / 97$, flow rate $=1.0 \mathrm{~mL} / \mathrm{min}$, $\left.\mathrm{t}=25{ }^{\circ} \mathrm{C}, \lambda=210 \mathrm{~nm}\right) \mathrm{t}_{\mathrm{R}}=10.8 \mathrm{~min}$ (major), $12.8 \mathrm{~min}$ (minor). ${ }^{1} \mathbf{H} \mathbf{N M R}\left(400 \mathrm{MHz}, \mathrm{CDCl}_{3}\right) \delta 7.21(\mathrm{dd}, J$ $=7.6,1.6 \mathrm{~Hz}, 1 \mathrm{H}), 7.17-7.11(\mathrm{~m}, 2 \mathrm{H}), 7.05(\mathrm{td}, J=7.2,1.2 \mathrm{~Hz}, 1 \mathrm{H}), 2.59(\mathrm{q}, J=7.2 \mathrm{~Hz}, 1 \mathrm{H}), 2.32(\mathrm{~s}, 3 \mathrm{H})$, $1.32(\mathrm{~d}, J=7.6 \mathrm{~Hz}, 3 \mathrm{H}), 1.23(\mathrm{~s}, 6 \mathrm{H}), 1.22(\mathrm{~s}, 6 \mathrm{H}) .{ }^{13} \mathrm{C} \mathbf{N M R}\left(101 \mathrm{MHz}, \mathrm{CDCl}_{3}\right) \delta 143.4,135.6,130.0$, 127.1, 126.0, 125.0, 83.2, 24.7, 24,6, 19.9, 16.4. The NMR spectra were in accordance with those reported in the literature. ${ }^{24}$

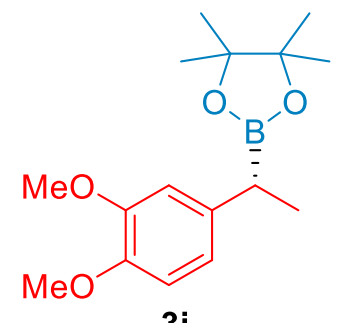

$3 \mathbf{i}$

(R)-2-(1-(3,4-dimethoxyphenyl)ethyl)-4,4,5,5-tetramethyl-1,3,2-dioxaborolane (3i):

Prepared according to the general procedure from 3,4-dimethoxystyrene $\mathbf{1 i}$ (41.0 $\mathrm{mg}$, $0.25 \mathrm{mmol}$ ) and $\mathrm{B}_{2} \mathrm{pin}_{2} 2(127.0 \mathrm{mg}, 0.5 \mathrm{mmol})$ using $2.5 \mathrm{~mL}$ toluene. The crude reaction was purified by flash column chromatography using hexanes/EtOAc $=10: 1$ as the eluent to afford $3 \mathbf{i}$ as a colorless oil in $50 \%$ yield $(36.5 \mathrm{mg}, 0.125 \mathrm{mmol})$. Optical rotation: $[\alpha]_{\mathrm{D}}{ }^{24}=-1.80^{\circ}\left(\mathrm{c} 0.66, \mathrm{CHCl}_{3}\right)$. The enantiomeric excess ( $84 \%$ ee) was determined after stereospecifically oxidized to the corresponding alcohol by HPLC (CHIRALPAK ASH, $i$ PrOH/hexane $=$ $5 / 95$, flow rate $=1.0 \mathrm{~mL} / \mathrm{min}, \mathrm{t}=25^{\circ} \mathrm{C}, \lambda=220 \mathrm{~nm}$ ) $\mathrm{t}_{\mathrm{R}}=15.2 \mathrm{~min}$ (minor), 18.4 min (major). ${ }^{1} \mathbf{H} \mathbf{N M R}$ $\left(400 \mathrm{MHz}, \mathrm{CDCl}_{3}\right) \delta 6.79-6.73(\mathrm{~m}, 3 \mathrm{H}), 3.86(\mathrm{~s}, 3 \mathrm{H}), 3.84(\mathrm{~s}, 3 \mathrm{H}), 2.37(\mathrm{q}, J=7.2 \mathrm{~Hz}, 1 \mathrm{H}), 1.30(\mathrm{~d}, J=$ 
7.6 Hz, 3H), 1.21 (s, 6H), 1.20 (s, 6H). ${ }^{13} \mathrm{C}$ NMR (101 MHz, $\left.\mathrm{CDCl}_{3}\right) \delta 148.6,146.6,137.5,119.3,111.21$, 111.20, 83.2, 55.8, 55.7, 24.6 (two peaks overlapped), 17.3. The NMR spectra were in accordance with those reported in the literature. ${ }^{25}$

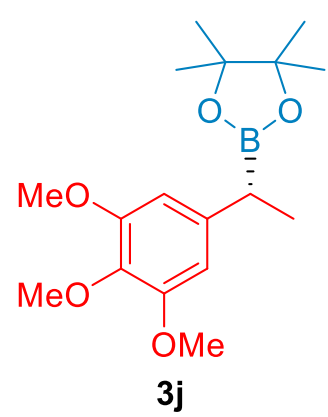

(R)-2-(1-(3,4,5-trimethoxyphenyl)ethyl)-4,4,5,5-tetramethyl-1,3,2-dioxaborolane (3j): Prepared according to the general procedure from 3,4,5-trimethoxystyrene $\mathbf{1 j}$ (48.5 mg, $0.25 \mathrm{mmol}$ ) and $\mathrm{B}_{2} \operatorname{pin}_{2} 2(127.0 \mathrm{mg}, 0.5 \mathrm{mmol})$ using $2.5 \mathrm{~mL}$ toluene. The crude reaction was purified by flash column chromatography using hexanes/EtOAc $=$ 10:1 as the eluent to afford $\mathbf{3 j}$ as a colorless oil in $53 \%$ yield $(42.7 \mathrm{mg}, 0.133 \mathrm{mmol})$. Optical rotation: $[\alpha]_{\mathrm{D}}^{24}=-1.98^{\circ}\left(\mathrm{c} 0.66, \mathrm{CHCl}_{3}\right)$. The enantiomeric excess $(78 \%$ ee $)$ was determined after stereospecifically oxidized to the corresponding alcohol by HPLC $\left(\right.$ CHIRALPAK ASH, $i$ PrOH $/$ hexane $=2 / 98$, flow rate $\left.=1.0 \mathrm{~mL} / \mathrm{min}, \mathrm{t}=25^{\circ} \mathrm{C}, \lambda=220 \mathrm{~nm}\right) \mathrm{t}_{\mathrm{R}}=13.4$ $\min$ (minor), $14.2 \min$ (major). ${ }^{1} \mathbf{H}$ NMR (400 MHz, $\left.\mathrm{CDCl}_{3}\right) \delta 6.44(\mathrm{~s}, 2 \mathrm{H}), 3.84(\mathrm{~s}, 6 \mathrm{H}), 3.81(\mathrm{~s}, 3 \mathrm{H}), 2.36$ $(\mathrm{q}, J=7.2 \mathrm{~Hz}, 1 \mathrm{H}), 1.31(\mathrm{~d}, J=7.6 \mathrm{~Hz}, 3 \mathrm{H}), 1.23(\mathrm{~s}, 6 \mathrm{H}), 1.21(\mathrm{~s}, 6 \mathrm{H}) .{ }^{13} \mathbf{C} \mathbf{N M R}\left(101 \mathrm{MHz}, \mathrm{CDCl}_{3}\right) \delta$ 153.0, 140.7, 135.5, 104.6, 83.3, 60.8, 55.9, 24.7, 24.6, 17.3. ${ }^{11} \mathbf{B}$ NMR (193 MHz, $\left.\mathrm{CDCl}_{3}\right)$ 33.5. HRMS (ESI) m/z: $[\mathrm{M}+\mathrm{H}]^{+}$Calcd for $\mathrm{C}_{17} \mathrm{H}_{28} \mathrm{BO}_{5}$ 323.2024; found: 323.2036 .

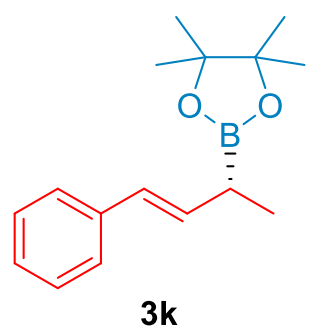

$(1 R, 2 E)-2-(4-p h e n y l b u t-3-e n-2-y l)-4,4,5,5$-tetramethyl-1,3,2-dioxaborolane $\quad(3 \mathrm{k})$ : Prepared according to the general procedure from trans-1-phenyl-1,3-butadiene $1 \mathbf{k}$ (32.5 mg, $0.25 \mathrm{mmol}$ ) and $\mathrm{B}_{2} \mathrm{pin}_{2} 2(127.0 \mathrm{mg}, 0.5 \mathrm{mmol})$ using $2.5 \mathrm{~mL}$ toluene. The crude reaction was purified by flash column chromatography using hexanes/EtOAc $=$ $10: 1$ as the eluent to afford $\mathbf{3 k}$ as a colorless oil in $60 \%$ yield $(38.7 \mathrm{mg}, 0.15 \mathrm{mmol})$. Optical rotation: $[\alpha]_{\mathrm{D}}{ }^{24}=-1.57^{\circ}\left(\mathrm{c} 0.63, \mathrm{CHCl}_{3}\right)$. The enantiomeric excess $(67 \%$ ee $)$ was determined after stereospecifically oxidized to the corresponding alcohol by HPLC (CHIRALPAK IG, $i \mathrm{PrOH} /$ hexane $=3 / 97$, flow rate $=1.0 \mathrm{~mL} / \mathrm{min}, \mathrm{t}=25^{\circ} \mathrm{C}, \lambda=210 \mathrm{~nm}$ ) $\mathrm{t}_{\mathrm{R}}=17.5 \mathrm{~min}$ (minor), $18.8 \mathrm{~min}$ (major). ${ }^{1} \mathbf{H}$ NMR $\left(400 \mathrm{MHz}, \mathrm{CDCl}_{3}\right) \delta$ 7.36-7.34 (m, 2H), 7.30-7.25 (m, 2H), 7.18-7.14 (m, 1H), $6.35-$ $6.34(\mathrm{~m}, 2 \mathrm{H}), 2.09-2.03(\mathrm{~m}, 1 \mathrm{H}), 1.24(\mathrm{~s}, 12 \mathrm{H}), 1.19(\mathrm{~d}, J=7.2 \mathrm{~Hz}, 3 \mathrm{H}) .{ }^{13} \mathbf{C} \mathbf{N M R}\left(101 \mathrm{MHz}, \mathrm{CDCl}_{3}\right) \delta$ 138.3, 133.3, 128.4, 127.6, 126.5, 125.9, 83.3, 24.7, 24.6, 14.8. The NMR spectra were in accordance with those reported in the literature. ${ }^{26}$ 


\section{Scalability}

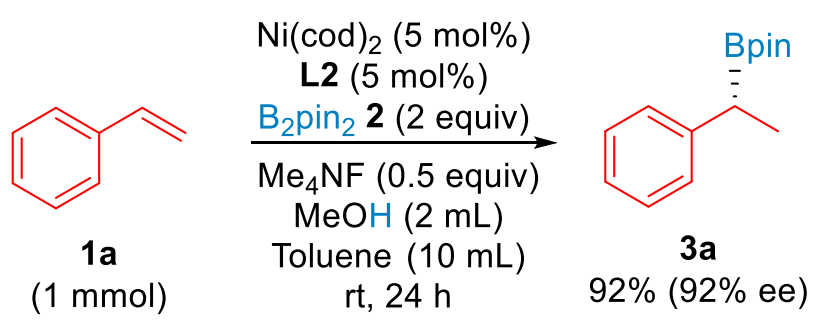

In a nitrogen-filled glovebox, an oven dried 20 -mL scintillation vial was charged with $\mathrm{Ni}(\operatorname{cod})_{2}(14 \mathrm{mg}$, $0.05 \mathrm{mmol}$ ), L2 (18.8 mg, $0.05 \mathrm{mmol}$ ), anhydrous Me $\mathrm{N}_{4} \mathrm{NF}$ (46.4 mg, $0.5 \mathrm{mmol}$ ), and $\mathrm{B}_{2} \mathrm{pin}_{2} 2$ (508 mg, 2 $\mathrm{mmol})$. Methanol $(2 \mathrm{~mL})$ and toluene $(10 \mathrm{~mL})$ were then added, and the resulting mixture was gently stirred at room temperature until clear. Next, styrene $1 \mathbf{a}(104 \mathrm{mg}, 1 \mathrm{mmol})$ was added and the vial was sealed and taken out of the glovebox. The reaction mixture was stirred at room temperature for 24 hours. Upon completion, the reaction mixture was filtered through a short plug of silica gel eluting with DCM and concentrated under reduced pressure. The crude product was purified by flash column chromatography using hexanes/EtOAc $=30: 1$ as the eluent to give the hydroboration product 3a as a colorless oil in $92 \%$ yield (213 mg, $0.92 \mathrm{mmol})$. Optical rotation: $[\alpha]_{\mathrm{D}}{ }^{24}=-1.52^{\circ}\left(\mathrm{c} 0.70, \mathrm{CHCl}_{3}\right)$. The enantiomeric excess $(92 \%$ ee) was determined after stereospecifically oxidized to the corresponding alcohol by HPLC (CHIRALCEL $\mathrm{ODH}, i \mathrm{PrOH} / \mathrm{hexane}=5 / 95$, flow rate $=1.0 \mathrm{~mL} / \mathrm{min}, \mathrm{t}=25^{\circ} \mathrm{C}, \lambda=220 \mathrm{~nm}$ ) $\mathrm{t}_{\mathrm{R}}=8.2 \mathrm{~min}$ (major), $8.9 \min$ (minor).

\section{Synthetic Applications}
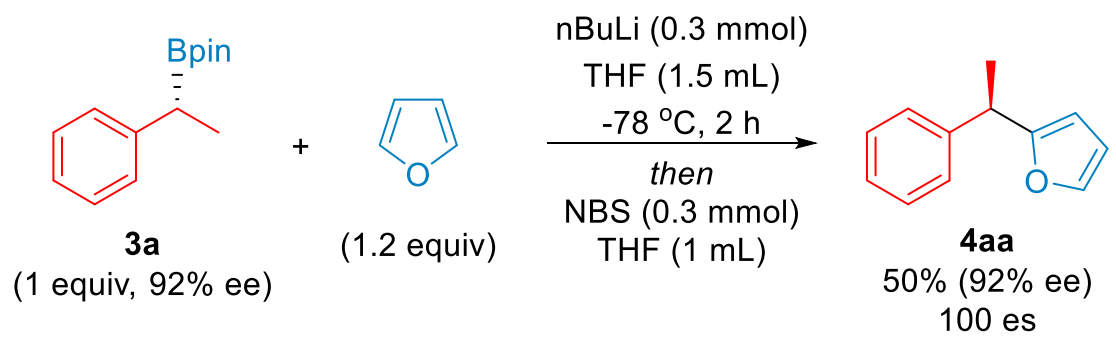

(R)-2-(1-Phenylethyl)furan (4aa): Prepared according to the reported literature procedure by Aggarwal and co-workers: ${ }^{27} \mathrm{In}$ a $25-\mathrm{mL}$ round bottom flask under nitrogen, a solution of furan (20.4 $\left.\mathrm{mg}, 0.3 \mathrm{mmol}\right)$ in THF $(1 \mathrm{~mL})$ was cooled to $-78^{\circ} \mathrm{C}$ and treated with $\mathrm{nBuLi}(2.5 \mathrm{M}$ in hexanes, $0.12 \mathrm{~mL})$. The reaction was allowed to warm to room temperature and stirred for $1 \mathrm{~h}$. The mixture was then cooled to $-78{ }^{\circ} \mathrm{C}$ and a solution of $3 \mathbf{a}(58 \mathrm{mg}, 0,25 \mathrm{mmol}, 92 \%$ ee) in THF $(0.5 \mathrm{~mL})$ was added dropwise, and the reaction was stirred at $-78{ }^{\circ} \mathrm{C}$ for $1 \mathrm{~h}$. Next, a solution of N-bromosuccinimide (NBS, $53.4 \mathrm{mg}, 0.3 \mathrm{mmol}$ ) in THF (1 $\mathrm{mL}$ ) was added dropwise. After being stirred for $1 \mathrm{~h}$ at $-78{ }^{\circ} \mathrm{C}$, a saturated aqueous solution of $\mathrm{Na}_{2} \mathrm{~S}_{2} \mathrm{O}_{3}$ was added and the reaction mixture was allowed to warm to room temperature. The reaction mixture was diluted 
with $\mathrm{Et}_{2} \mathrm{O}$ and water. The organic layer was collected, and the aqueous layer was extracted with $\mathrm{Et}_{2} \mathrm{O}$. The combined organic layers were dried over $\mathrm{MgSO}_{4}$, filtered, and concentrated under reduce pressure. The crude product was purified by flash column chromatography using hexanes/ $\mathrm{Et}_{2} \mathrm{O}=50: 1$ as the eluent to give the product $4 \mathbf{a a}$ as a colorless oil in $50 \%$ yield $\left(21.5 \mathrm{mg},(0.125 \mathrm{mmol})\right.$. Optical rotation: $[\alpha]_{\mathrm{D}}{ }^{24}=-$ $1.90^{\circ}$ (c $0.55, \mathrm{CHCl}_{3}$ ). The enantiomeric excess (92\% ee, $100 \%$ es) was determined by HPLC $\left(\right.$ CHIRALCEL OJH, $i \operatorname{PrOH} /$ hexane $=10 / 90$, flow rate $\left.=1.0 \mathrm{~mL} / \mathrm{min}, \mathrm{t}=25^{\circ} \mathrm{C}, \lambda=220 \mathrm{~nm}\right) \mathrm{t}_{\mathrm{R}}=16.0 \mathrm{~min}$ (major), $17.5 \mathrm{~min}$ (minor). The racemic product was synthesized by a reported literature procedure. ${ }^{28}{ }^{1} \mathbf{H}$ NMR $\left(400 \mathrm{MHz}, \mathrm{CDCl}_{3}\right) \delta$ 7.33-7.29 (m, 3H), 7.24-7.21 (m, 3H), 6.30 (dd, $\left.J=3.2,2 \mathrm{~Hz}, 1 \mathrm{H}\right), 6.06$ (dt, $J$ $=3.2,0.8 \mathrm{~Hz}, 1 \mathrm{H}), 4.13(\mathrm{q}, J=7.2 \mathrm{~Hz}, 1 \mathrm{H}), 1.60(\mathrm{~d}, J=7.2 \mathrm{~Hz}, 3 \mathrm{H}) .{ }^{13} \mathbf{C} \mathbf{N M R}\left(101 \mathrm{MHz}, \mathrm{CDCl}_{3}\right) \delta 159.0$, $144.2,141.3,128.5,127.3,126.5,109.9,104.9,39.2,20.5$. The NMR spectra were in accordance with those reported in the literature. ${ }^{27}$

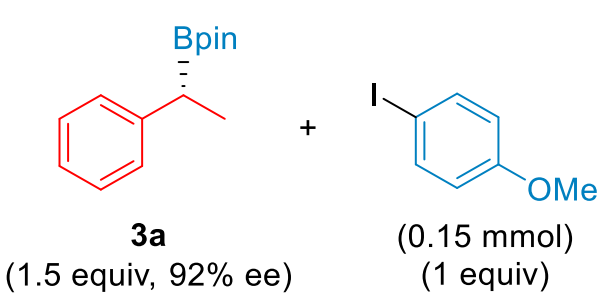

$$
\begin{gathered}
\mathrm{Pd}\left(\mathrm{PPh}_{3}\right)_{4}(7 \mathrm{~mol} \%) \\
\mathrm{PPh}_{3}(5 \mathrm{~mol} \%) \\
\underset{\mathrm{Ag}_{2} \mathrm{O}(1.8 \text { equiv })}{\longrightarrow} \\
\mathrm{K}_{2} \mathrm{CO}_{3}(1.5 \text { equiv }) \\
\mathrm{DME}(3 \mathrm{~mL}) \\
85^{\circ} \mathrm{C}, 24 \mathrm{~h}
\end{gathered}
$$

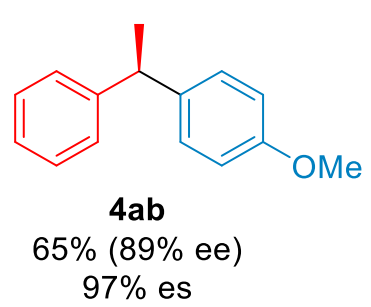

(R)-1-methoxy-4-(1-phenylethyl)benzene (4ab): Prepared according to the reported literature procedure by Crudden and co-workers: ${ }^{29}$ In a nitrogen-filled glovebox, an oven dried 1-dram vial was charged with $\mathrm{Pd}\left(\mathrm{PPh}_{3}\right)_{4}(13.5 \mathrm{mg}, 0.0117 \mathrm{mmol}), \mathrm{PPh}_{3}(12.3 \mathrm{mg}, 0.0470 \mathrm{mmol}) \mathrm{Ag}_{2} \mathrm{O}$ (52.3 mg, $\left.0.226 \mathrm{mmol}\right), \mathrm{K}_{2} \mathrm{CO}_{3}$ (32.1 mg, $0.232 \mathrm{mmol}$ ), 4-iodoanisole (35.1 mg, $0.150 \mathrm{mmol})$, and $\mathbf{3 a}(52.7 \mathrm{mg}, 0.227 \mathrm{mmol}, 92 \% \mathrm{ee})$. DME ( $3 \mathrm{~mL}$ ) was then added, and the reaction vial was sealed, taken out of the glovebox, and stirred at 85 ${ }^{\circ} \mathrm{C}$ in an aluminum reaction block (for 1-dram vials) for $24 \mathrm{~h}$. Upon completion, the mixture was cooled to room temperature and filtered through a plug of silica gel eluting with DCM and concentrated under reduced pressure. The crude product was purified by flash column chromatography using hexanes/ $\mathrm{Et}_{2} \mathrm{O}=50: 1$ as the eluent to give the product $\mathbf{4 a b}$ as a colorless oil in $65 \%$ yield $(20.7 \mathrm{mg},(0.975 \mathrm{mmol})$. Optical rotation: $[\alpha]_{\mathrm{D}}^{24}=-1.41^{\circ}\left(\mathrm{c} 0.65, \mathrm{CHCl}_{3}\right)$. The enantiomeric excess (89\% ee, 97\% es) was determined by HPLC $\left(\right.$ CHIRALCEL OJH, $i$ PrOH $/$ hexane $=10 / 90$, flow rate $\left.=1.0 \mathrm{~mL} / \mathrm{min}, \mathrm{t}=25^{\circ} \mathrm{C}, \lambda=220 \mathrm{~nm}\right) \mathrm{t}_{\mathrm{R}}=18.0 \mathrm{~min}$ (minor), 19.4 min (major). The racemic product was synthesized by a reported literature procedure. ${ }^{28}{ }^{1} \mathbf{H}$ NMR (400 MHz, $\left.\mathrm{CDCl}_{3}\right) \delta$ 7.33-7.29 (m, 2H), 7.26-7.17 (m, 5H), $6.86(\mathrm{~d}, J=8.4 \mathrm{~Hz}, 2 \mathrm{H}), 4.15(\mathrm{~d}, J=$ $7.2 \mathrm{~Hz}, 1 \mathrm{H}), 3.80(\mathrm{~s}, 3 \mathrm{H}), 1.65(\mathrm{~d}, J=7.2 \mathrm{~Hz}, 3 \mathrm{H}) .{ }^{13} \mathbf{C} \mathbf{N M R}\left(101 \mathrm{MHz}, \mathrm{CDCl}_{3}\right) \delta 157.8,146.8,138.6$, $128.5,128.4,127.6,126.0,113.7,55.2,44.0,22.1$. The NMR spectra were in accordance with those reported in the literature. ${ }^{29}$ 


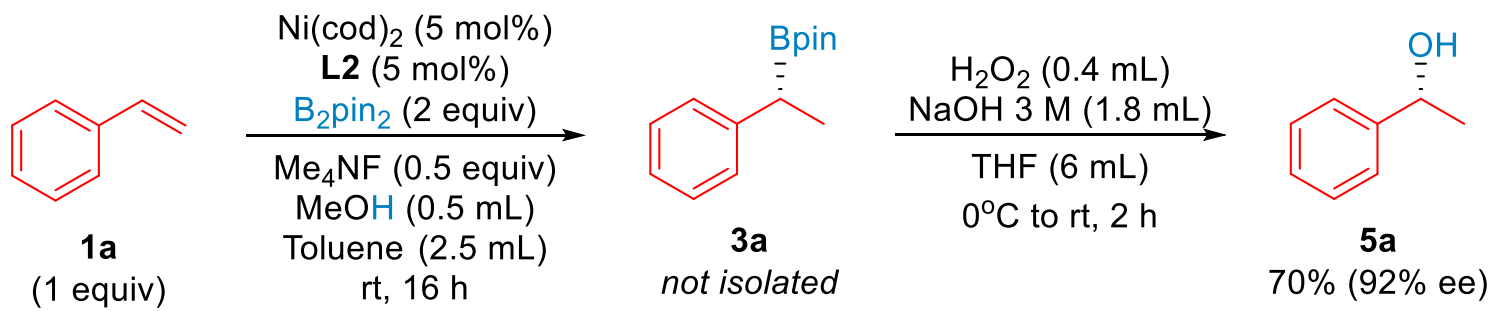

(R)-1-phenylethan-1-ol (5a): In a nitrogen-filled glovebox, an oven dried 1-dram vial was charged with $\mathrm{Ni}(\mathrm{cod})_{2}(3.5 \mathrm{mg}, 0.0125 \mathrm{mmol}), \mathbf{L 2}(4.7 \mathrm{mg}, 0.0125 \mathrm{mmol})$, anhydrous $\mathrm{Me}_{4} \mathrm{NF}$ (11.6 mg, $\left.0.125 \mathrm{mmol}\right)$, and $\mathrm{B}_{2} \operatorname{pin}_{2} 2(127 \mathrm{mg}, 0.5 \mathrm{mmol})$. Methanol $(0.5 \mathrm{~mL})$ and toluene $(2.5 \mathrm{~mL})$ were then added, and the resulting mixture was gently stirred at room temperature until clear. Next, styrene 1a (26 mg, $0.25 \mathrm{mmol})$ was added and the vial was sealed and taken out of the glovebox. The reaction mixture was stirred at room temperature for 16 hours. Upon completion, the reaction mixture was filtered through a short plug of silica gel eluting with DCM and concentrated under reduced pressure. The crude boronate ester 3a was used directly for the next step without further purification. Under air, the crude 3a was dissolved in THF (6 mL) and the solution was cooled to $0{ }^{\circ} \mathrm{C}$ in an ice-water bath. Then, aqueous $\mathrm{NaOH} 3 \mathrm{M}(1.8 \mathrm{~mL})$ and $\mathrm{H}_{2} \mathrm{O}_{2}(0.4$ $\mathrm{mL}$ ) were sequentially added. The resulting mixture was allowed to warm to room temperature and stirred for $2 \mathrm{~h}$. Upon completion, the reaction mixture was diluted with $\mathrm{H}_{2} \mathrm{O}(10 \mathrm{~mL})$ and extracted with EtOAc (3 $\times 10 \mathrm{~mL}$ ). The combined organic layers were washed with brine, dried over anhydrous $\mathrm{Na}_{2} \mathrm{SO}_{4}$, filtered, and concentrated under reduced pressure. The crude product was purified by flash column chromatography using hexanes/EtOAc $=10 / 1$ as the eluent to give the alcohol 5a as a colorless oil in 70\% yield (21.4 mg, 0.18 mmol). Optical rotation: $[\alpha]_{\mathrm{D}}{ }^{24}=-1.98^{\circ}\left(\mathrm{c} 0.85, \mathrm{CHCl}_{3}\right)$. The enantiomeric excess $(92 \%$ ee $)$ was determined by HPLC (CHIRALCEL ODH, $i \mathrm{PrOH} / \mathrm{hexane}=5 / 95$, flow rate $=1.0 \mathrm{~mL} / \mathrm{min}, \mathrm{t}=25^{\circ} \mathrm{C}, \lambda=$ $220 \mathrm{~nm}$ ) $\mathrm{t}_{\mathrm{R}}=9.0 \mathrm{~min}$ (major), $9.8 \mathrm{~min}$ (minor). ${ }^{1} \mathbf{H} \mathbf{N M R}\left(400 \mathrm{MHz}, \mathrm{CDCl}_{3}\right) \delta$ 7.39-7.33 (m, 4H), 7.30-7.26 $(\mathrm{m}, 1 \mathrm{H}), 4.89$ (q, $J=6.4 \mathrm{~Hz}, 1 \mathrm{H}), 2.02(\mathrm{~s}, 1 \mathrm{H}), 1.49(\mathrm{~d}, J=6.4 \mathrm{~Hz}, 3 \mathrm{H}) .{ }^{13} \mathbf{C} \mathbf{~ N M R}\left(101 \mathrm{MHz}, \mathrm{CDCl}_{3}\right) \delta$ $145.9,128.6,127.6,125.5,70.5,25.2$. The NMR spectra were in accordance with those reported in the literature. $^{30}$

\section{Kinetic Studies}

In a nitrogen-filled glovebox, an oven dried 1-dram vial was charged with $\mathrm{Ni}(\mathrm{cod})_{2}(3.5 \mathrm{mg}, 0.0125 \mathrm{mmol})$, L2 (4.7 mg, $0.0125 \mathrm{mmol}$ ), anhydrous $\mathrm{Me}_{4} \mathrm{NF}$ (11.6 mg, $0.125 \mathrm{mmol}$ ), and $\mathrm{B}_{2} \operatorname{pin}_{2} 2$ (127 mg, $0.5 \mathrm{mmol}$ ). Methanol $(0.5 \mathrm{~mL})$ and toluene $(2.5 \mathrm{~mL})$ were then added, and the resulting mixture was gently stirred at room temperature until clear. Next, styrene $1 \mathbf{a}(26 \mathrm{mg}, 0.25 \mathrm{mmol})$ was added and the vial was sealed and taken out of the glovebox. The reaction mixture was stirred at room temperature for 20 minutes. Upon completion, the reaction mixture was filtered through a short plug of silica gel eluting with DCM and 
concentrated under reduced pressure. The crude reaction mixture was dissolved in $\mathrm{CDCl}_{3}(1 \mathrm{~mL})$ and $\mathrm{CH}_{2} \mathrm{Br}_{2}(8.8 \mu \mathrm{L}, 0.125 \mathrm{mmol})$ was added as an internal standard. An NMR yield was determined by ${ }^{1} \mathrm{H}$ NMR spectroscopy of the crude reaction mixture. Three additional reactions with the same condition were also set up and stirred at room temperature for 40 minutes, 60 minutes and 80 minutes. Upon completion, the reactions were filtered through short plugs of silica gel eluting with DCM, concentrated under reduced pressure, and determined ${ }^{1} \mathrm{H}$ NMR yields with $\mathrm{CH}_{2} \mathrm{Br}_{2}$ as an internal standard.

For the reaction set with $\mathrm{MeOD}$, four parallel reactions were set up and stirred at room temperature for 20 minutes, 40 minutes, 60 minutes, and 80 minutes. Reaction conditions were the same as the reactions in methanol, but $\mathrm{MeOD}(0.5 \mathrm{~mL})$ was used in place of $\mathrm{MeOH}$. Upon completion, the reactions were filtered through short plugs of silica gel eluting with DCM, concentrated under reduced pressure, and determined ${ }^{1} \mathrm{H}$ NMR yields with $\mathrm{CH}_{2} \mathrm{Br}_{2}$ as an internal standard.
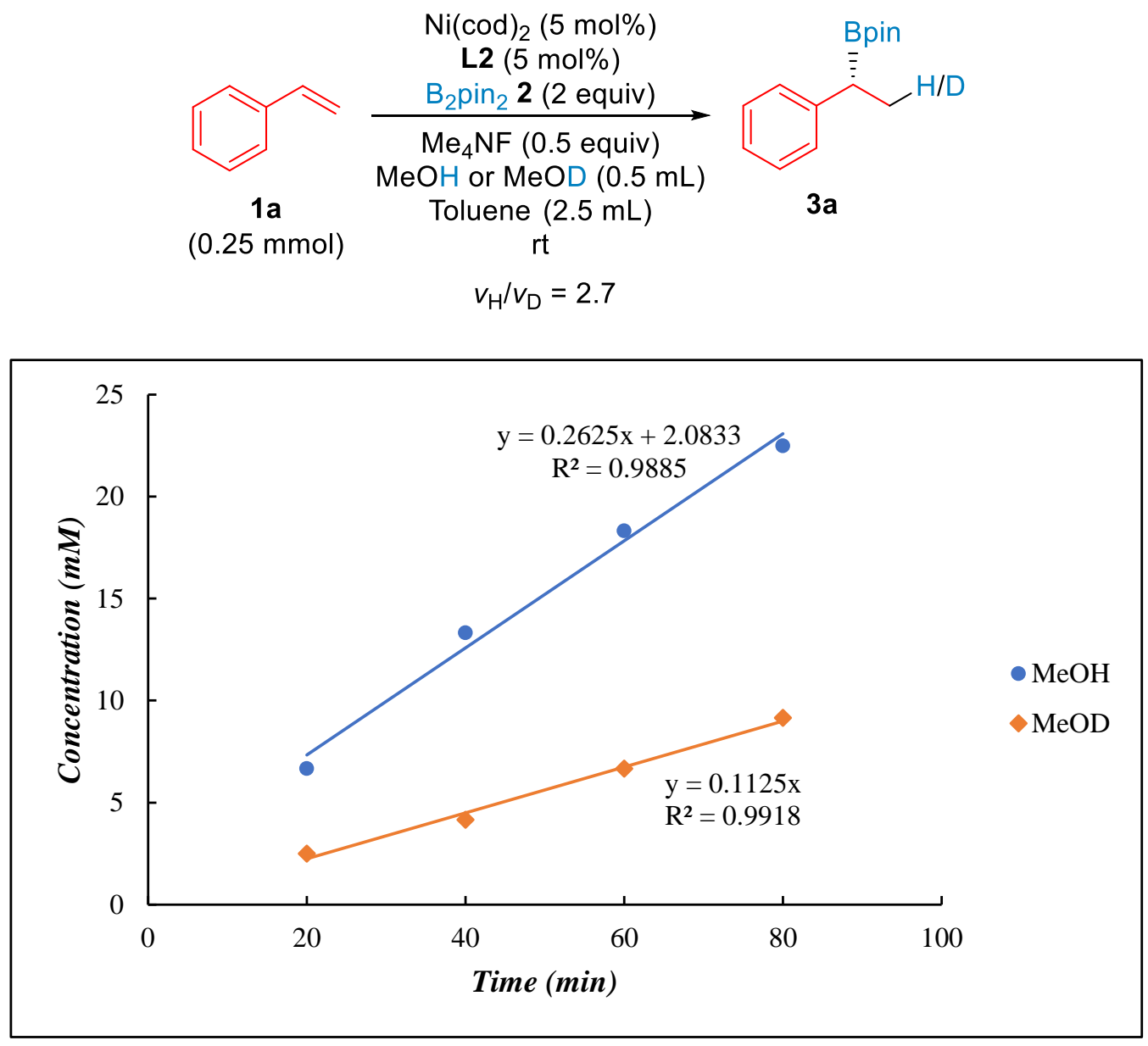


\section{Protodeboronation Test}

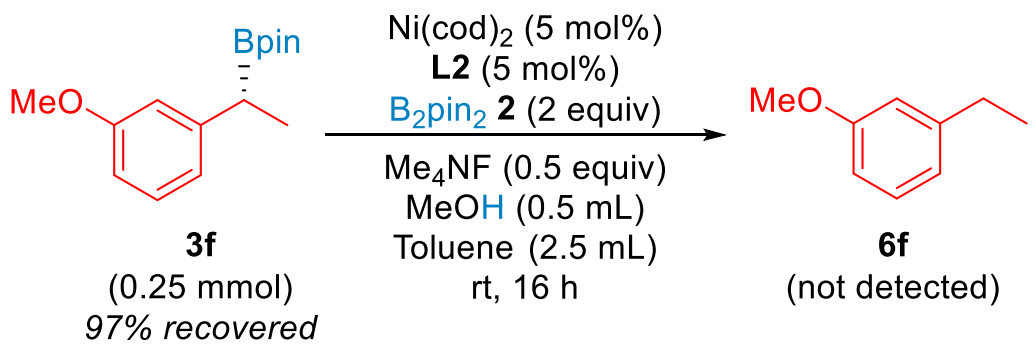

In a nitrogen-filled glovebox, an oven dried 1-dram vial was charged with $\mathrm{Ni}(\mathrm{cod})_{2}(3.5 \mathrm{mg}, 0.0125 \mathrm{mmol})$, $\mathbf{L 2}$ (4.7 mg, $0.0125 \mathrm{mmol}$ ), anhydrous $\mathrm{Me}_{4} \mathrm{NF}$ (11.6 mg, $\left.0.125 \mathrm{mmol}\right)$, and $\mathrm{B}_{2} \mathrm{pin}_{2} 2$ (127 mg, $\left.0.5 \mathrm{mmol}\right)$. Methanol $(0.5 \mathrm{~mL})$ and toluene $(1.5 \mathrm{~mL})$ were then added, and the resulting mixture was gently stirred at room temperature until clear. Next, benzylic boronate ester $\mathbf{3 f}(65.5 \mathrm{mg}, 0.25 \mathrm{mmol})$ was added and the vial was sealed and taken out of the glovebox. The reaction mixture was stirred at room temperature for $16 \mathrm{~h}$. Upon completion, the reaction mixture was filtered through a short plug of silica gel eluting with DCM and concentrated under reduced pressure. The crude reaction mixture was dissolved in $\mathrm{CDCl}_{3}(1 \mathrm{~mL})$ and $\mathrm{CH}_{2} \mathrm{Br}_{2}(8.8 \mu \mathrm{L}, 0.125 \mathrm{mmol})$ was added as an internal standard. An NMR yield was determined by ${ }^{1} \mathrm{H}$ NMR spectroscopy of the crude reaction mixture, with $97 \%$ of $\mathbf{3 f}$ was determined and there was no formation of protodeboronation product $\mathbf{6 f}$.
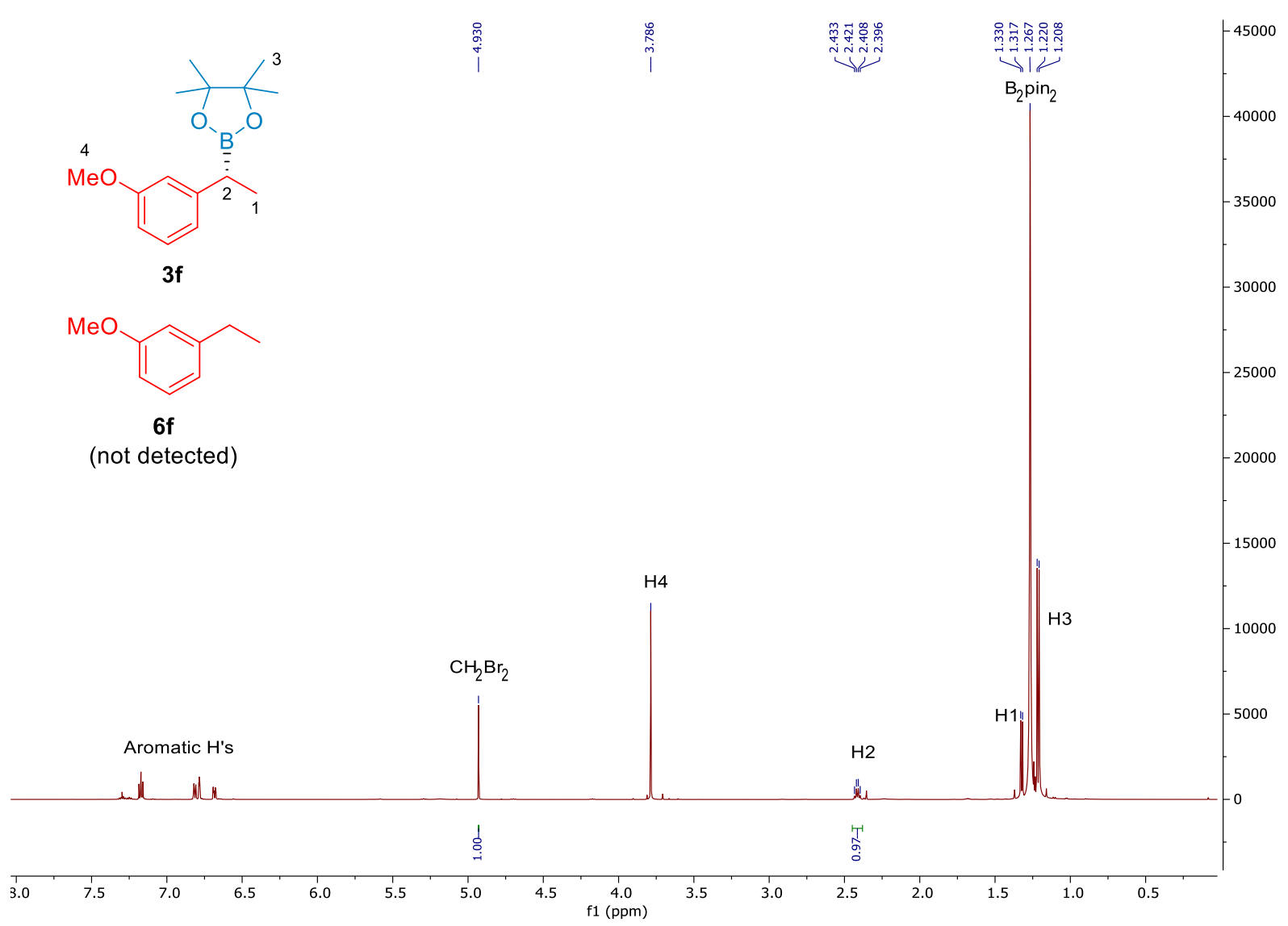


\section{References}

1. Hu, N.; Jung, H.; Zheng, Y.; Lee, J.; Zhang, L.; Ullah, Z.; Xie, X.; Harms, K.; Baik, M.-H.; Meggers, E. Angew. Chem. Int. Ed. 2018, 57, 6242-6246.

2. Chu, L.; Qing, F.-L. Org. Lett. 2010, 12, 5060-5063.

3. Deguest, G.; Bischoff, L.; Fruit, C.; Marsais, F. Org. Lett. 2007, 9, 1165-1167.

4. Nomura, E.; Hosoda, A.; Mori, H.; Taniguchi, H. Green Chem. 2005, 7, 863-866.

5. Faler, C. A.; Joullié, M. M. Org. Lett. 2007, 9, 1987-1990.

6. Zheng, M.; Huang, L.; Wu, W.; Jiang, H. Org. Lett. 2013, 15, 1838-1841.

7. Tran, H. N.; Burgett, R. W.; Stanley, L. M. J. Org. Chem. 2021, 86, 3836-3849.

8. Wu, H.; Wang, Q.; Zhu, J. Angew. Chem., Int. Ed. 2018, 57, 2721-2725.

9. Liu, C.; Yi, J.-C.; Liang, X.-W.; Xu, R.-Q.; Dai, L.-X.; You, S.-L. Chem.-Eur. J. 2016, 22, 1081310816.

10. Bera, S.; Mao, R.; Hu, X. Nat. Chem. 2021, 13, 270-277.

11. Boechat, N.; Clark, J. H. J. Chem. Soc. Chem. Commun. 1993, 921-922.

12. Estopiñá-Durán, S.; Mclean, E. B.; Donnelly, L. J.; Hockin, B. M.; Taylor, J. E. Org. Lett. 2020, 22, $7547-7551$.

13. Ian P. Query, I. P.; Squier, P. A.; Larson, E. M.; Isley, N. A.; Clark, T. B. J. Org. Chem. 2011, 76, 6452-6456.

14. Liu, S.; Wolf, C. Org. Lett. 2007, 9, 2965-2968.

15. Schaaf, P.; Gojic, V.; Bayer, T.; Rudroff, F.; Schnürch, M.; Mihovilovic, M. D. ChemCatChem 2018, 10, 920-924.

16. Castro, L. C. M.; Bézier, D.; Sortais, J.-B.; Darcel, C. Adv. Synth. Catal. 2011, 353, 1279-1284.

17. Hofmann, L. E; Hofmann, D.; Prusko, L.; Altmann, L.-M.; Heinrich, M. R. Adv. Synth. Catal. 2020, 362, 1485-1489.

18. Unsworth, P. J.; Leonori, D.; Aggarwal, V. K. Angew. Chem. Int. Ed. 2014, 53, 9846-9850.

19. Wang, Z.; Bachman, S.; Dudnik, A. S.; Fu, G. C. Angew. Chem. Int. Ed. 2018, 57, 14529-14532.

20. Basch, C. H.; Cobb, K. M.; Watson, M. P. Org. Lett. 2016, 18, 136-139.

21. Chen, X.; Cheng, Z.; Lu, Z. ACS Catal. 2019, 9, 4025-4029.

22. Ulm, F.; Cornaton, Y.; Djukic, J.-P.; Chetcuti, M. J.; Ritleng, V. Chem. Eur. J. 2020, 26, 8916-8925.

23. Yang, C.; Gao, Y.; Bai, S.; Jiang, C.; Qi, X. J. Am. Chem. Soc. 2020, 142, 11506-11513.

24. Hu, J.; Sun, H.; Cai, W.; Pu, X.; Zhang, Y.; Shi, Z. J. Org. Chem. 2016, 81, 14-24.

25. Peng, J.; Docherty, J. H.; Dominey, A. P.; Thomas, S. P. Chem. Commun. 2017, 53, 4726-4729.

26. Zhou, Q.; Srinivas, H. D.; Zhang, S.; Watson, M. P. J. Am. Chem. Soc. 2016, 138, 11989-11995. 
27. Odachowski, M.; Bonet, A.; Essafi, S.; Conti-Ramsden, P.; Harvey, J. N.; Leonori, D.; Aggarwal, V. K. J. Am. Chem. Soc. 2016, 138, 9521-953223.

28. Xiao, L.-J.; Cheng, L.; Feng, W.-M.; Li, M.-L.; Xie, J.-H.; Zhou, Q.-L. Angew. Chem., Int. Ed. 2018, $57,461-464$.

29. Glasspoole, B. W.; Oderinde, M. S.; Moore, B. D.; Antoft-Finch, A.; Crudden, C. M. Synthesis 2013, $45,1759-1763$.

30. Xiao, J.; Wong, Z. Z.; Lu, Y. P.; Loh, T. P. Adv. Synth. Catal. 2010, 352, 1107-1112. 


\section{HPLC Charts of Chiral Products}

\begin{tabular}{|llll|}
\hline \multicolumn{2}{c|}{ S A M P E } & IN F O R M A T I O N \\
\hline \hline Sample Name: & & Acquired By: & System \\
Sample Type: & Unknown & Sample Set Name HT_08052021 \\
Vial: & 45 & Acq. Method Set: & 3_ODH 95_5 1mpm \\
Injection \#: & 1 & Processing Method Hydroboration \\
Injection Volume: & $5.00 \mathrm{ul}$ & Channel Name: & W2489 ChB \\
Run Time: & 15.0 Minutes & Proc. Chnl. Descr.: W2489 ChB 220nm \\
& & & \\
Date Acquired: & $8 / 5 / 20219: 39: 31$ AM CDT & & \\
Date Processed: & $8 / 5 / 20213: 28: 02$ PM CDT & & \\
\hline
\end{tabular}

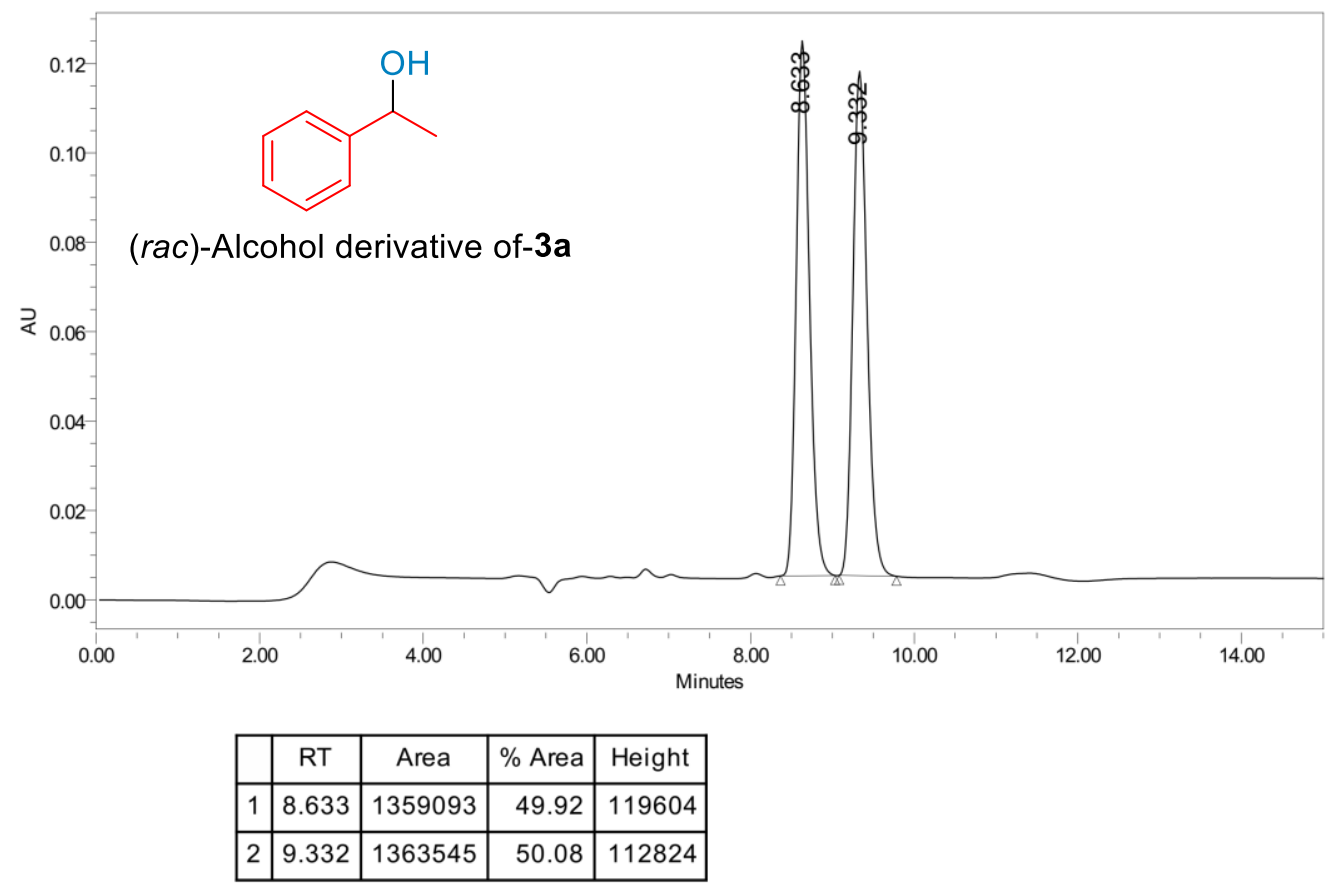

Reported by User: System

Project Name: Stanley_1IStanley4

Report Method: Default Individual Report

Date Printed:

Report Method I[ 1014

$8 / 5 / 2021$

Page: 1 of 1

3:28:38 PM US/Central 


\begin{tabular}{|llll|}
\hline & \multicolumn{2}{c|}{ S A M P E } & I N F O R M A T I O N \\
\hline \hline Sample Name: & & Acquired By: & System \\
Sample Type: & Unknown & Sample Set Name & HT_08052021 \\
Vial: & 47 & Acq. Method Set: & 3_ODH 95_5 1mpm \\
Injection \#: & 1 & Processing Method Hydroboration \\
Injection Volume: & $5.00 \mathrm{ul}$ & Channel Name: & W2489 ChB \\
Run Time: & 15.0 Minutes & Proc. Chnl. Descr.: W2489 ChB 220nm \\
& & & \\
Date Acquired: & $8 / 5 / 20219: 55: 09$ AM CDT & & \\
Date Processed: & $8 / 5 / 20213: 27: 32$ PM CDT & & \\
\hline
\end{tabular}

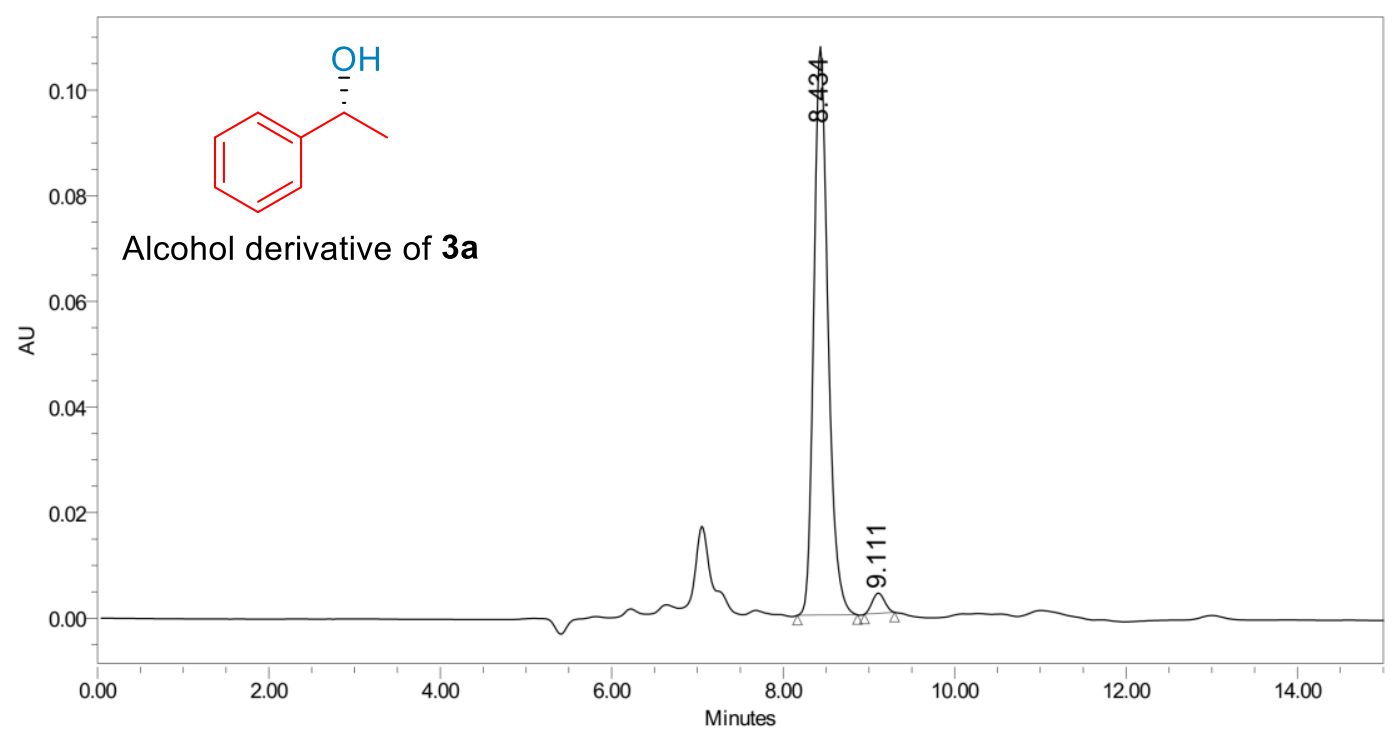

\begin{tabular}{|r|c|r|r|r|}
\hline & RT & \multicolumn{1}{c|}{ Area } & \% Area & Height \\
\hline 1 & 8.434 & 1236924 & 96.98 & 107607 \\
\hline 2 & 9.111 & 38515 & 3.02 & 3837 \\
\hline
\end{tabular}

Reported by User: System

Report Method: Default Individual Report Report Method I[ 1014

Page: 1 of 1
Project Name: Stanley_1\Stanley4

Date Printed:

$8 / 5 / 2021$

3:30:04 PM US/Central 


\begin{tabular}{|llll|}
\hline & \multicolumn{2}{c|}{ S A M P E } & I N F O R M A T I O N \\
\hline \hline Sample Name: & Unknown & Acquired By: & System \\
Sample Type: & 46 & Sample Set Name & HT_08052021 \\
Vial: & 1 & Acq. Method Set: 3_ODH 95_5 1mpm \\
Injection \#: & Processing Method Hydroboration \\
Injection Volume: & $5.00 \mathrm{ul}$ & Channel Name: & W2489 ChB \\
Run Time: & 15.0 Minutes & Proc. Chnl. Descr.: W2489 ChB 220nm \\
& & & \\
Date Acquired: & $8 / 5 / 2021$ 10:10:54 AM CDT & & \\
Date Processed: & $9 / 5 / 20212: 57: 38$ PM CDT & \\
\hline
\end{tabular}

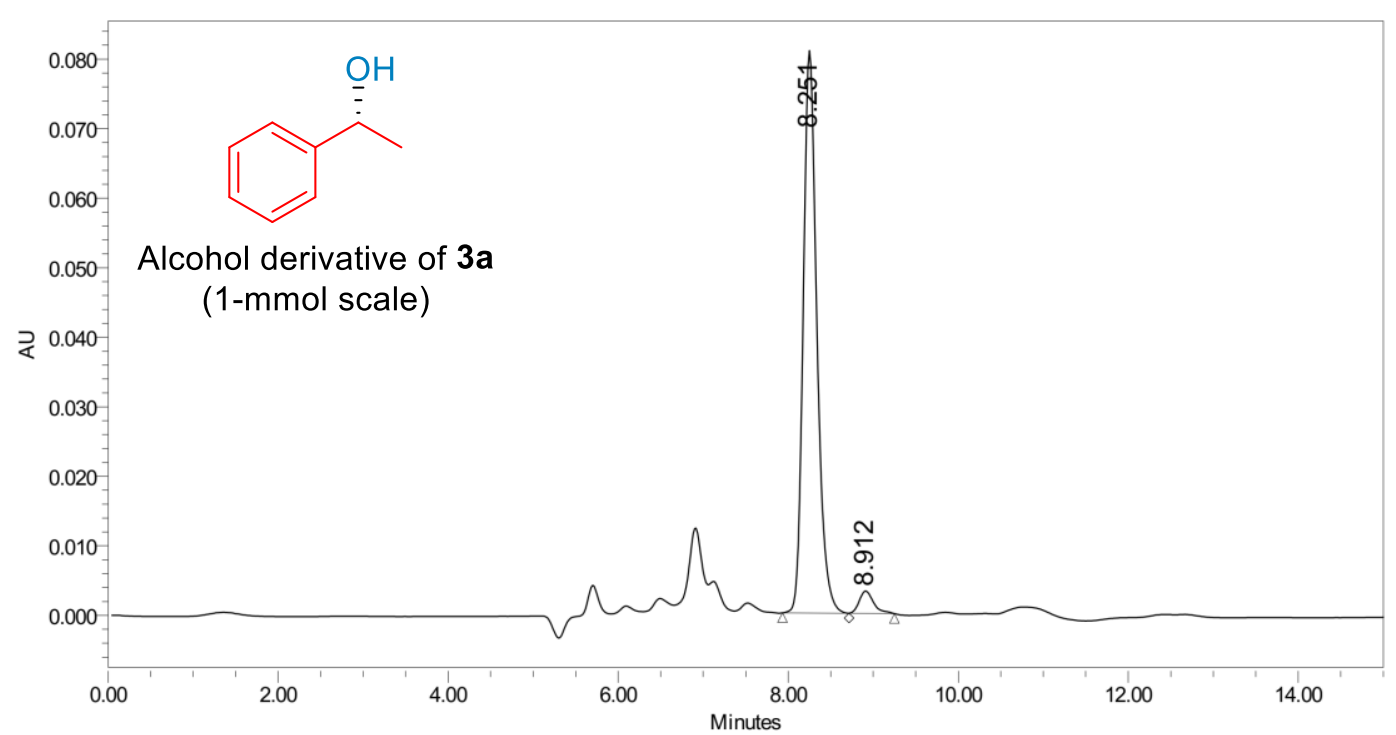

\begin{tabular}{|c|c|c|r|r|}
\hline & RT & Area & $\%$ Area & Height \\
\hline 1 & 8.251 & 902078 & 96.00 & 80824 \\
\hline 2 & 8.912 & 37564 & 4.00 & 3222 \\
\hline
\end{tabular}

Reported by User: System

Report Method: Default Individual Report Report Method I[ 1014

Page: 1 of 1
Project Name: Stanley_1\Stanley4

Date Printed:

$9 / 5 / 2021$

2:58:33 PM US/Central 


\begin{tabular}{|llll|}
\hline & \multicolumn{2}{c|}{ S A M P E } & I N F O R M A T I O N \\
\hline \hline Sample Name: & Unknown & Acquired By: & System \\
Sample Type: & 37 & Sample Set Name HT_08302021 \\
Vial: & 1 & Acq. Method Set: 3_ODH 95_5 1mpm \\
Injection \#: & Processing Method Hydroboration \\
Injection Volume: & 10.00 ul & Channel Name: & W2489 ChB \\
Run Time: & 15.0 Minutes & Proc. Chnl. Descr.: W2489 ChB 220nm \\
& & & \\
Date Acquired: & $8 / 30 / 20216: 49: 44$ PM CDT & & \\
Date Processed: & $8 / 31 / 20218: 04: 47$ AM CDT & \\
\hline
\end{tabular}

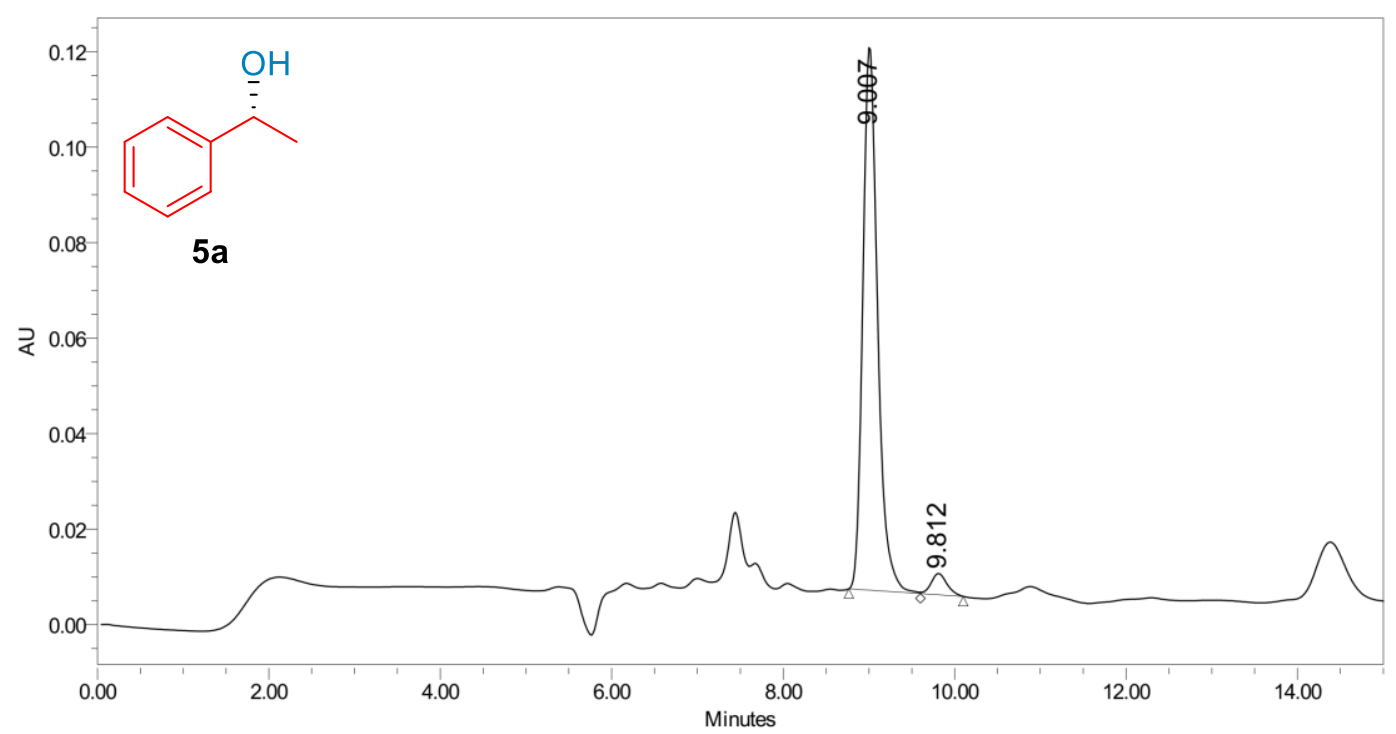

\begin{tabular}{|l|c|r|r|r|}
\hline & RT & \multicolumn{1}{|c|}{ Area } & \% Area & Height \\
\hline 1 & 9.007 & 1362291 & 95.99 & 114057 \\
\hline 2 & 9.812 & 56902 & 4.01 & 4459 \\
\hline
\end{tabular}

Reported by User: System

Report Method: Default Individual Report Report Method I[ 1014

Page: 1 of 1
Project Name: Stanley_1\Stanley4 Date Printed: $8 / 31 / 2021$ 8:05:20 AM US/Central 


\begin{tabular}{|c|c|c|c|}
\hline \multicolumn{2}{|r|}{ SAMPLE } & \multicolumn{2}{|c|}{ INFORMATION } \\
\hline Sample Name: & & Acquired By: & System \\
\hline Sample Type: & Unknown & Sample Set Name & HT_07032021_2 \\
\hline Vial: & 16 & Acq. Method Set: & 2_A A SH 95_5 1mpm \\
\hline Injection \#: & 1 & Processing Method & Hydroboration \\
\hline Injection Volume: & $5.00 \mathrm{ul}$ & Channel Name: & W2489 ChB \\
\hline Run Time: & 20.0 Minutes & Proc. Chnl. Descr.: & W2489 ChB 220nm \\
\hline Date Acquired: & 7/3/2021 11:45:26 AM CDT & & \\
\hline Date Processed: & 7/3/2021 12:11:18 PM CDT & & \\
\hline
\end{tabular}

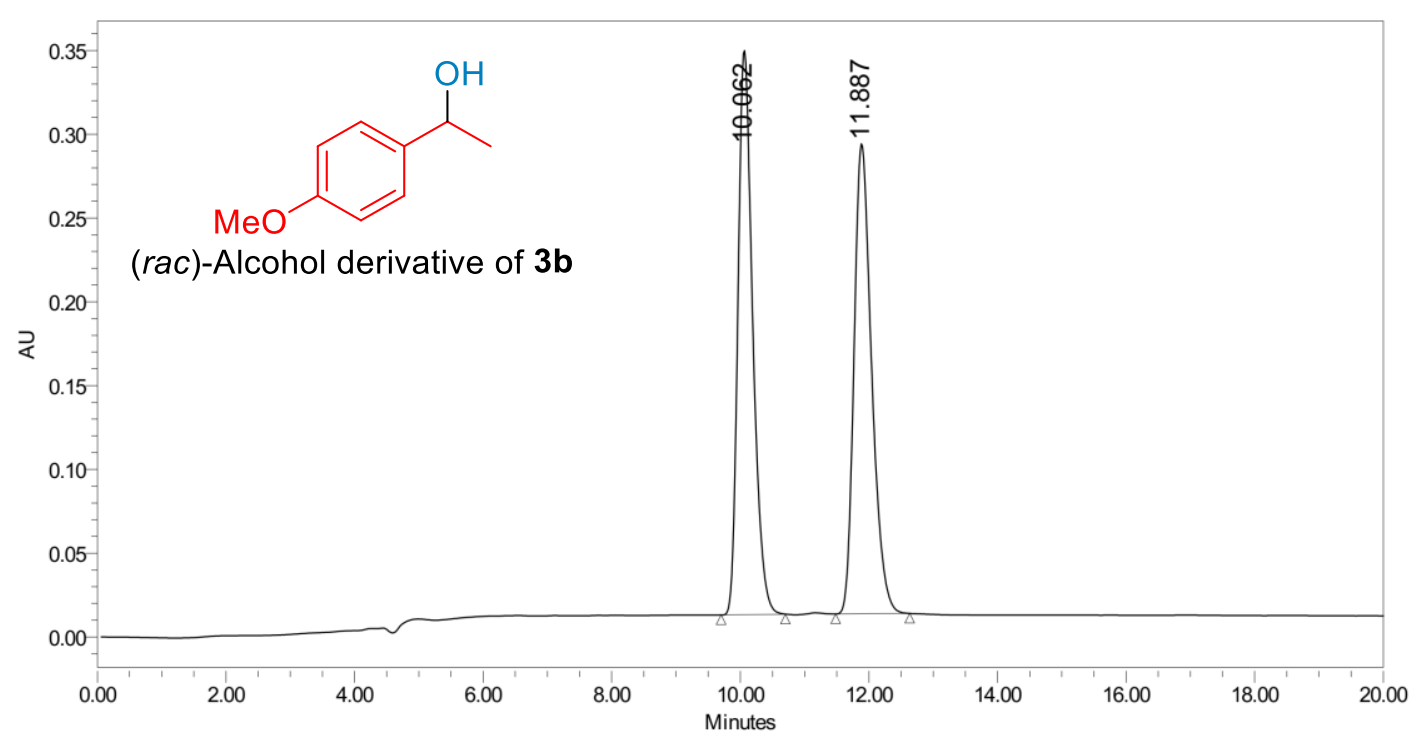

\begin{tabular}{|c|c|c|r|c|}
\hline & RT & Area & $\%$ Area & Height \\
\hline 1 & 10.062 & 5352697 & 50.11 & 336895 \\
\hline 2 & 11.887 & 5328909 & 49.89 & 280672 \\
\hline
\end{tabular}

Reported by User: System

Report Method: Default Individual Report Report Method I[ 1014

Page: 1 of 1
Project Name: Stanley_1\Stanley4

Date Printed:

$7 / 3 / 2021$

12:11:45 PM US/Central 


\begin{tabular}{|llll|}
\hline & \multicolumn{2}{c|}{ S A M P E } & IN F O R M A T I O N \\
\hline \hline Sample Name: & & Acquired By: & System \\
Sample Type: & Unknown & Sample Set Name & HT_07092021 \\
Vial: & 2 & Acq. Method Set: 2 ASH 95_5 1mpm \\
Injection \#: & 1 & Processing Method Hydroboration \\
Injection Volume: & 10.00 ul & Channel Name: & W2489 ChB \\
Run Time: & 20.0 Minutes & Proc. Chnl. Descr.: W2489 ChB 220nm \\
& & & \\
Date Acquired: & $7 / 9 / 20211: 12: 17$ PM CDT & & \\
Date Processed: & $7 / 9 / 20213: 12: 21$ PM CDT & & \\
\hline
\end{tabular}

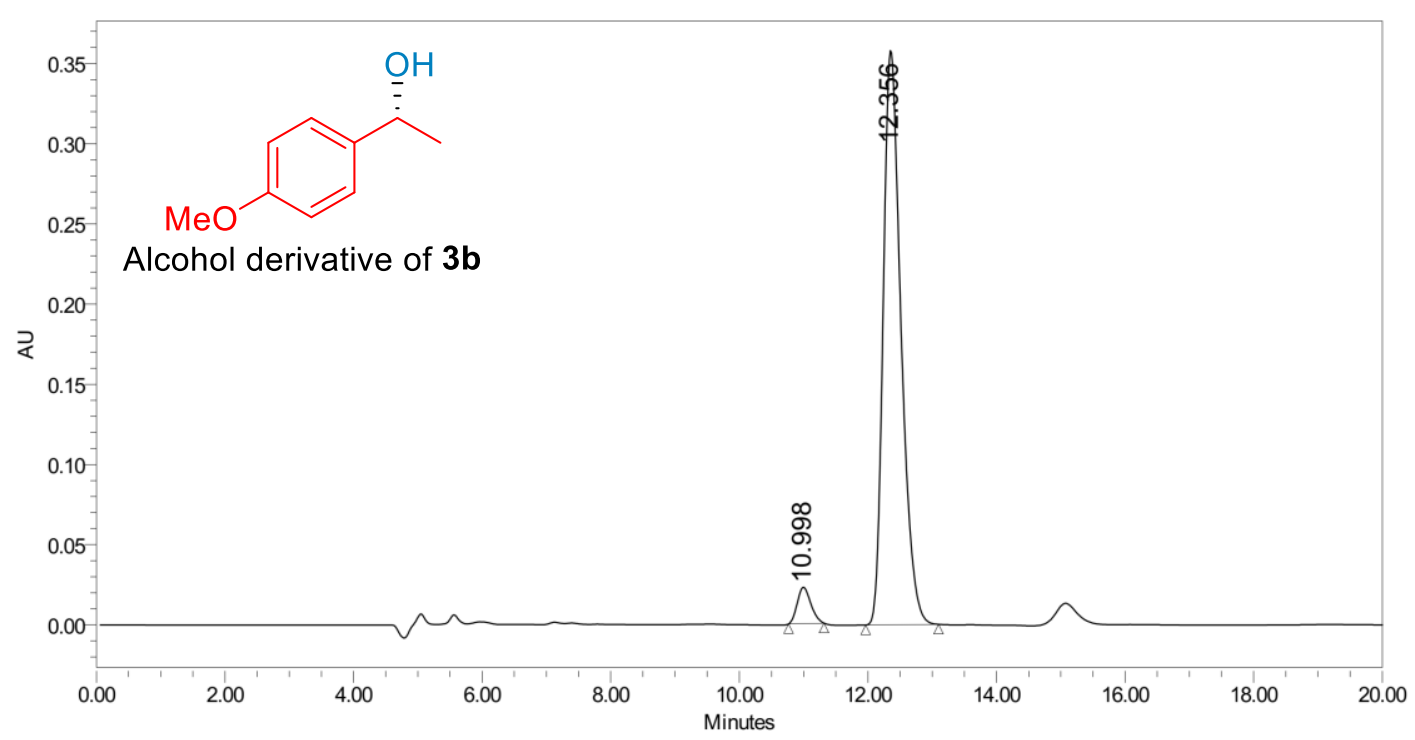

\begin{tabular}{|r|c|r|r|r|}
\hline & RT & Area & \% Area & Height \\
\hline 1 & 10.998 & 330879 & 4.59 & 22621 \\
\hline 2 & 12.356 & 6876589 & 95.41 & 358428 \\
\hline
\end{tabular}

Reported by User: System

Report Method: Default Individual Report Report Method I[ 1014

Page: 1 of 1
Project Name: Stanley_1\Stanley4

Date Printed:

$7 / 9 / 2021$

3:12:48 PM US/Central 


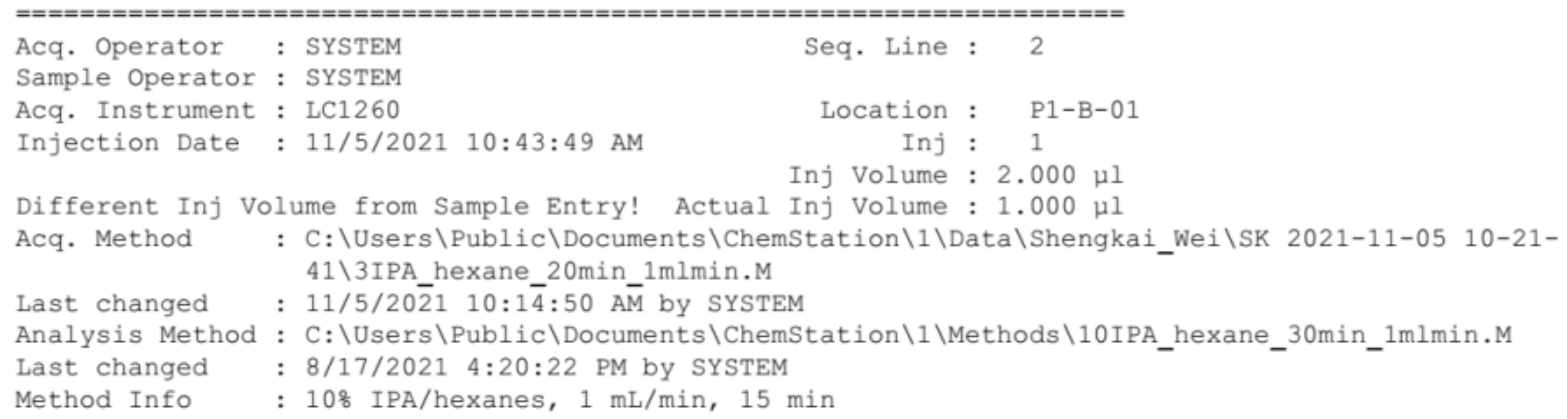<smiles>Cc1ccc(C(C)O)cc1</smiles>

\section{(rac)-Alcohol derivative of 3c}

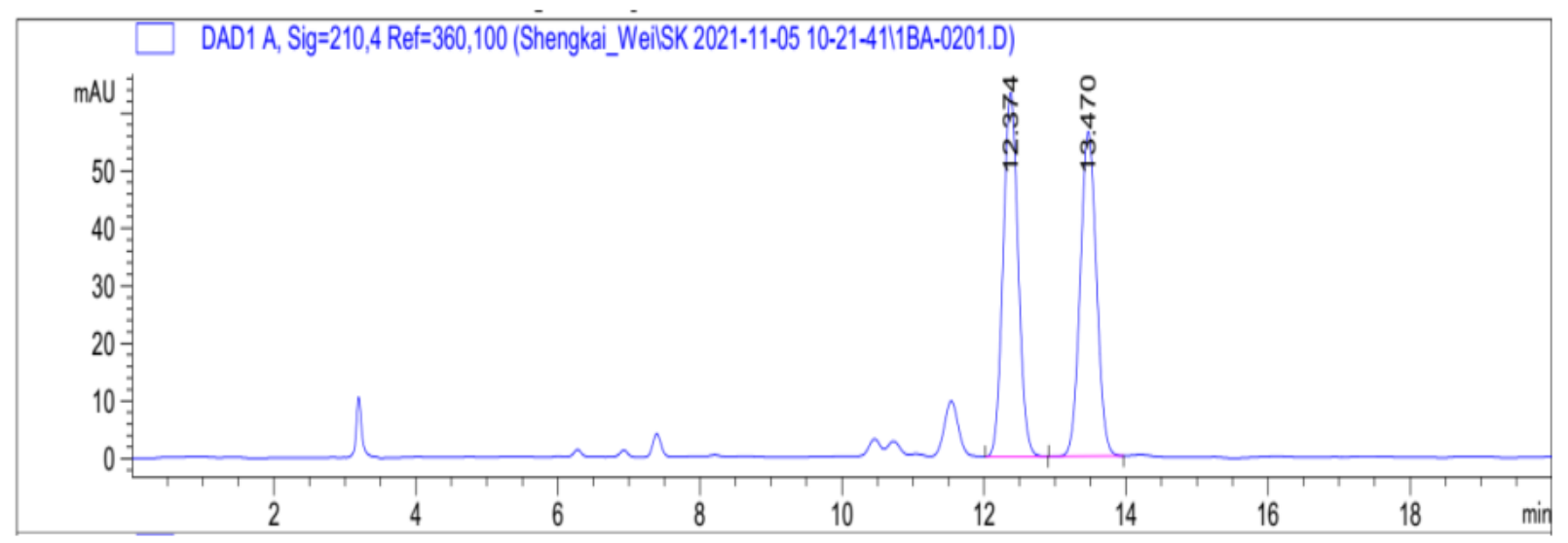

\begin{tabular}{|c|c|c|c|c|c|c|}
\hline $\begin{array}{c}\text { Peak } \\
\#\end{array}$ & $\begin{array}{c}\text { RetTime } \\
\text { [min] }\end{array}$ & Type & $\begin{array}{l}\text { Width } \\
\text { [min] }\end{array}$ & $\begin{array}{c}\text { Area } \\
{\left[\mathrm{mAU}^{\star} \mathrm{s}\right]}\end{array}$ & $\begin{array}{l}\text { Height } \\
\text { [mAU] }\end{array}$ & $\begin{array}{c}\text { Area } \\
\frac{8}{6}\end{array}$ \\
\hline \multicolumn{7}{|c|}{$----|------|----|-------|---------|---------|-------$} \\
\hline 1 & 12.374 & $\mathrm{BB}$ & 0.2295 & 930.16577 & 63.27373 & 50.9308 \\
\hline 2 & 13.470 & $\mathrm{BB}$ & 0.2493 & 896.16718 & 56.41636 & 49.0692 \\
\hline Tota & ls : & & & 1826.33295 & 119.69009 & \\
\hline
\end{tabular}




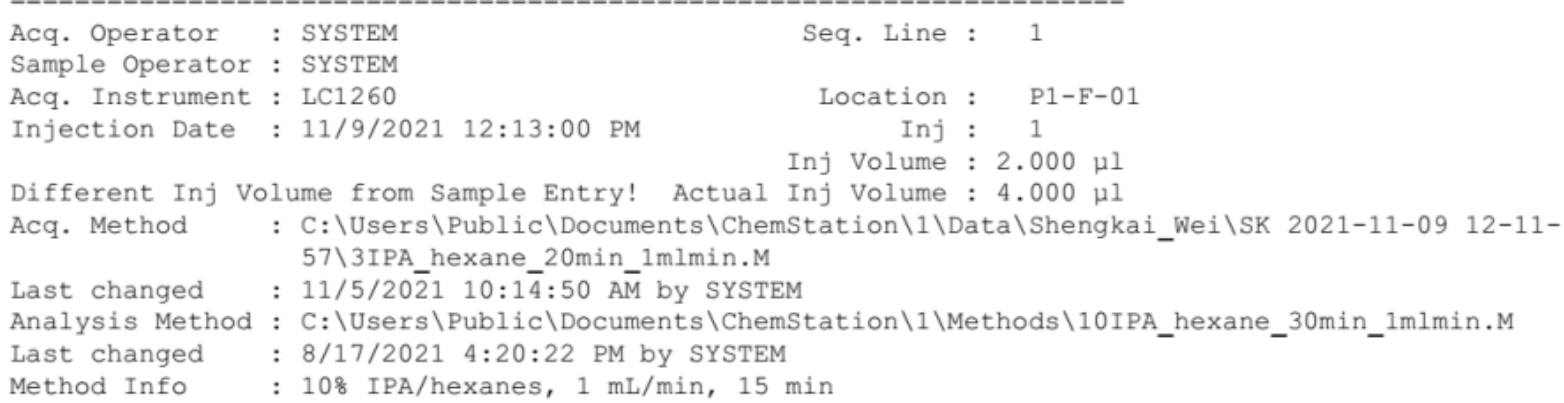<smiles>Cc1ccc([C@@H](C)O)cc1</smiles>

\section{Alcohol derivative of $\mathbf{3 c}$}

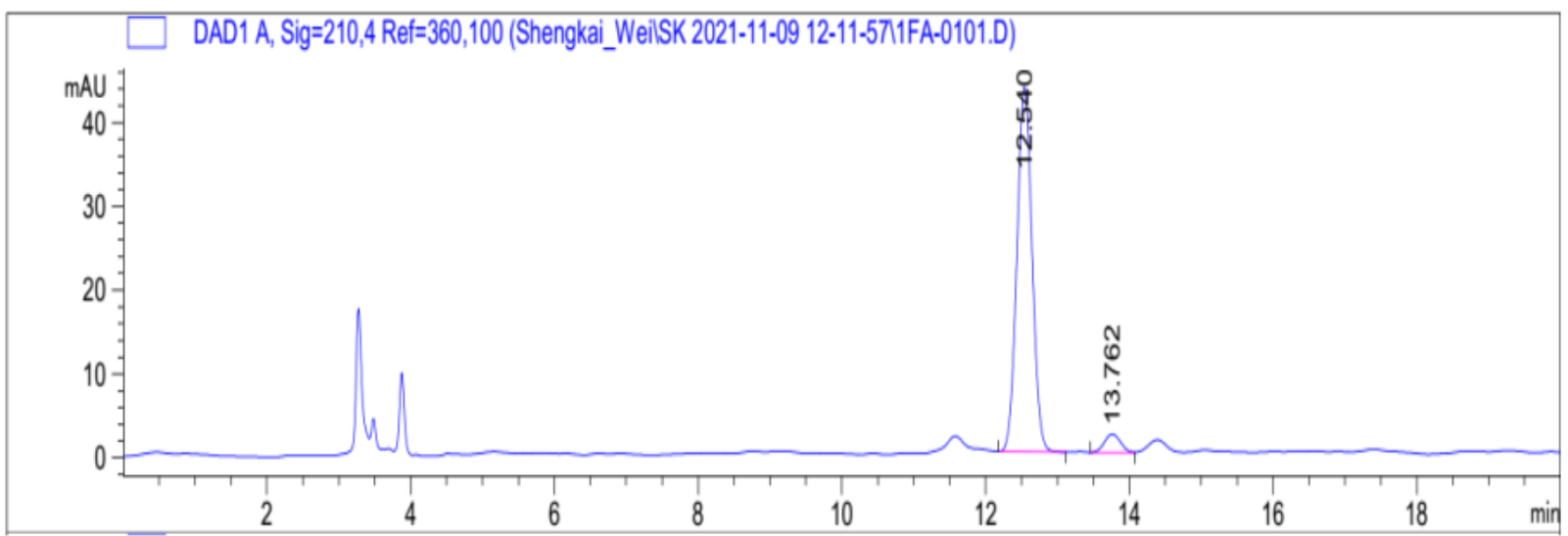

Signal 1: DAD1 A, Sig=210,4 Ref $=360,100$

\begin{tabular}{|c|c|c|c|c|c|c|}
\hline $\begin{array}{c}\text { Peak } \\
\#\end{array}$ & $\begin{array}{l}\text { RetTime } \\
\text { [min] }\end{array}$ & Type & $\begin{array}{l}\text { Width } \\
\text { [min] }\end{array}$ & $\begin{array}{c}\text { Area } \\
{\left[\mathrm{mAU}{ }^{\star} \mathrm{S}\right]}\end{array}$ & $\begin{array}{l}\text { Height } \\
\text { [mAU] }\end{array}$ & $\begin{array}{c}\text { Area } \\
8\end{array}$ \\
\hline \multicolumn{7}{|c|}{ 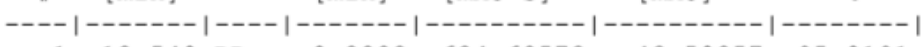 } \\
\hline 1 & 12.540 & $\mathrm{BB}$ & 0.2282 & 634.68573 & 43.50257 & 95.0191 \\
\hline 2 & 13.762 & BB & 0.2417 & 33.27002 & 2.18475 & 4.9809 \\
\hline Total & s : & & & 667.95575 & 45.68732 & \\
\hline
\end{tabular}


Acq. Operator : SYSTEM

Sample Operator : SYSTEM

Acq. Instrument : LC1260

Injection Date : 11/5/2021 10:22:41 AM

Seq. Line : 1

Location : $\mathrm{P} 1-\mathrm{A}-01$

Inj : 1

Inj Volume : $2.000 \mu 1$

Different Inj Volume from Sample Entry! Actual Inj Volume : $1.000 \mu 1$

Acq. Method : C: \Users \Public \Documents $\backslash$ ChemStation $\backslash 1 \backslash$ Data $\backslash$ Shengkai_Wei $\backslash S K ~ 2021-11-05$ 10-21$41 \backslash 3$ IPA hexane 20min $1 \mathrm{mlmin} . \mathrm{M}$

Last changed : $11 / 5 / 20 \overline{2} 1 \quad 10: 1 \overline{4}: 50$ AM by SYSTEM

Analysis Method : C: \Users \Public \Documents \ChemStation \1\Methods \10IPA hexane 30min 1mlmin.M

Last changed : $8 / 17 / 2021$ 4:20:22 PM by SYSTEM

Method Info : 10\% IPA/hexanes, $1 \mathrm{~mL} / \mathrm{min}, 15 \mathrm{~min}$

F<smiles>CC(O)c1ccc(F)cc1</smiles>

(rac)-Alcohol derivative of $\mathbf{3 d}$

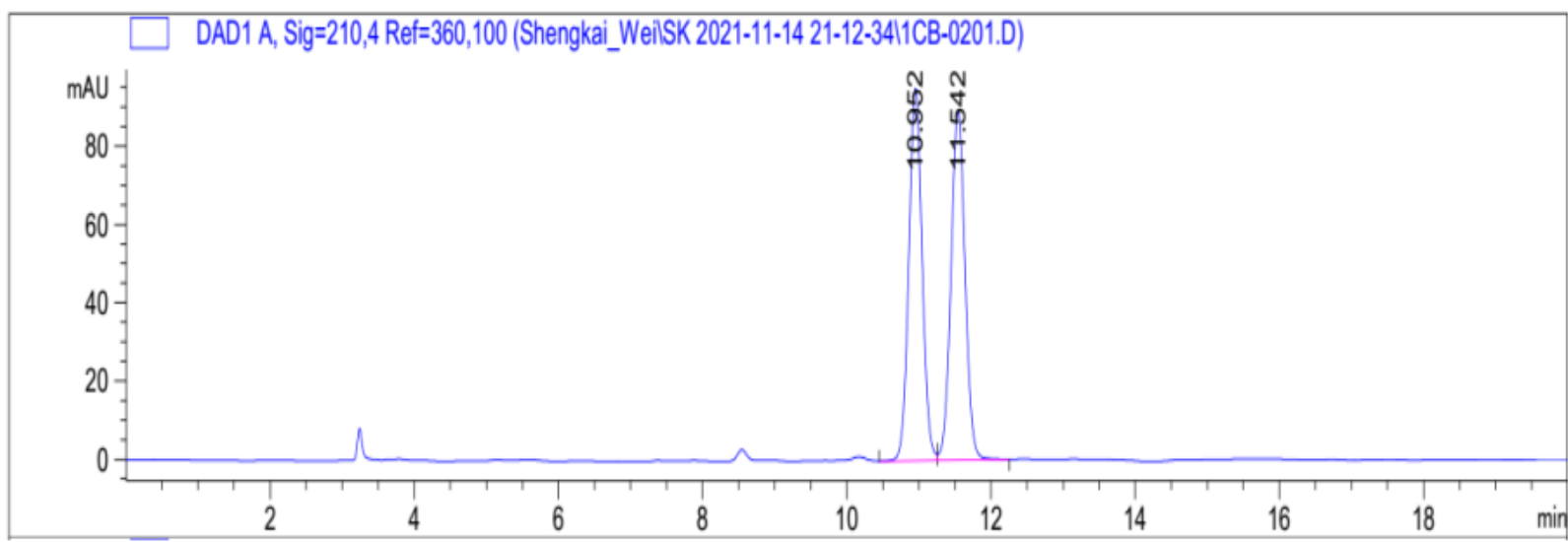

Signal 1: DAD1 A, Sig=210, 4 Ref $=360,100$

\begin{tabular}{|c|c|c|c|c|c|c|}
\hline $\begin{array}{c}\text { Peak } \\
\#\end{array}$ & $\begin{array}{l}\text { RetTime } \\
\text { [min] }\end{array}$ & Type & $\begin{array}{l}\text { Width } \\
\text { [min] }\end{array}$ & $\begin{array}{c}\text { Area } \\
{\left[\mathrm{mAU}{ }^{\star} \mathrm{S}\right]}\end{array}$ & $\begin{array}{l}\text { Height } \\
\text { [mAU] }\end{array}$ & $\begin{array}{c}\text { Area } \\
\frac{\circ}{6}\end{array}$ \\
\hline \multicolumn{7}{|c|}{ 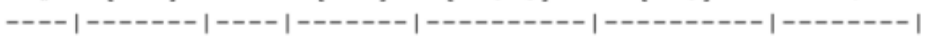 } \\
\hline 1 & 7.836 & BV & 0.1448 & 143.09636 & 15.34083 & 50.2349 \\
\hline 2 & 8.199 & VB & 0.1518 & 141.75789 & 14.53022 & 49.7651 \\
\hline To & . & & & 284.85425 & 87105 & \\
\hline
\end{tabular}


Acq. Operator : SYSTEM

Sample Operator : SYSTEM

Acq. Instrument : LC1260

Injection Date : 11/7/2021 3:33:25 PM
Seq. Line : 5

Location : P1-E-01

Inj : 1

Inj Volume : $2.000 \mu \mathrm{l}$

Different Inj Volume from Sample Entry! Actual Inj Volume : $1.000 \mu 1$

Acq. Method : C: \Users \Public \Documents \ChemStation $1 \backslash$ Data \Shengkai_Wei \SK 2021-11-07 12-5753\3IPA hexane $20 \mathrm{~min} 1 \mathrm{mlmin} . \mathrm{M}$

Last changed : $11 / 5 / 20 \overline{2} 1 \quad 10: 1 \overline{4}: 50$ AM by SYSTEM

Analysis Method : C: \Users \Public \Documents \ChemStation $1 \backslash$ Methods $\backslash 10 I P A$ hexane 30min $1 \mathrm{mlmin} . \mathrm{M}$

Last changed : 8/17/2021 4:20:22 PM by SYSTEM

Method Info : 10\% IPA/hexanes, $1 \mathrm{~mL} / \mathrm{min}, 15 \mathrm{~min}$<smiles>CC[C@@H](O)c1ccc(F)cc1</smiles>

\section{Alcohol derivative of $\mathbf{3 d}$}

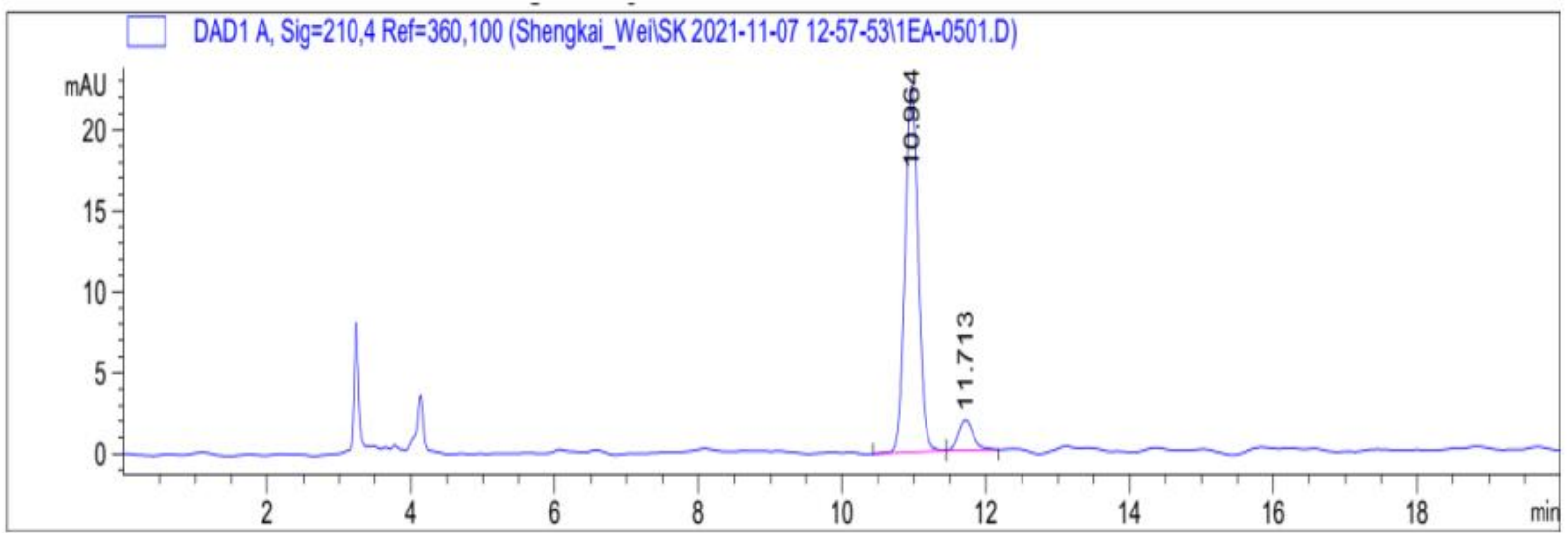

Signal 1: DAD1 A, Sig=210, 4 Ref $=360,100$

\begin{tabular}{|c|c|c|c|c|c|c|}
\hline $\begin{array}{c}\text { Peak } \\
\#\end{array}$ & $\begin{array}{c}\text { RetTime } \\
\text { [min] }\end{array}$ & Type & $\begin{array}{l}\text { Width } \\
\text { [min] }\end{array}$ & $\begin{array}{c}\text { Area } \\
{\left[\mathrm{mAU} \mathbf{U}^{*} \mathrm{~s}\right]}\end{array}$ & $\begin{array}{l}\text { Height } \\
\text { [mAU] }\end{array}$ & $\begin{array}{c}\text { Area } \\
\text { \% }\end{array}$ \\
\hline \multicolumn{7}{|c|}{ 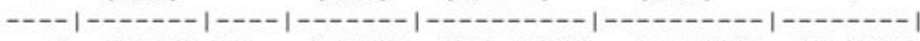 } \\
\hline 1 & 10.964 & $\mathrm{BB}$ & 0.1949 & 284.57639 & 22.60387 & 91.4325 \\
\hline 2 & 11.713 & $\mathrm{BB}$ & 0.2229 & 26.66557 & 1.84226 & 8.5675 \\
\hline Total & : & & & 311.24195 & 24.44613 & \\
\hline
\end{tabular}




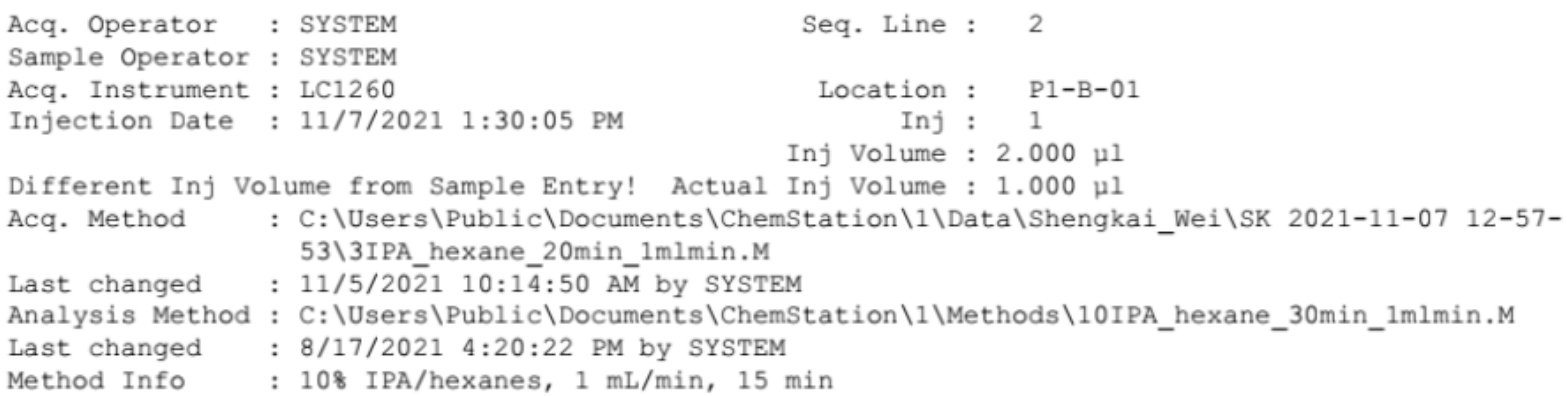<smiles>CC(O)c1ccc(C(F)(F)F)cc1</smiles>

(rac)-Alcohol derivative of $\mathbf{3 e}$

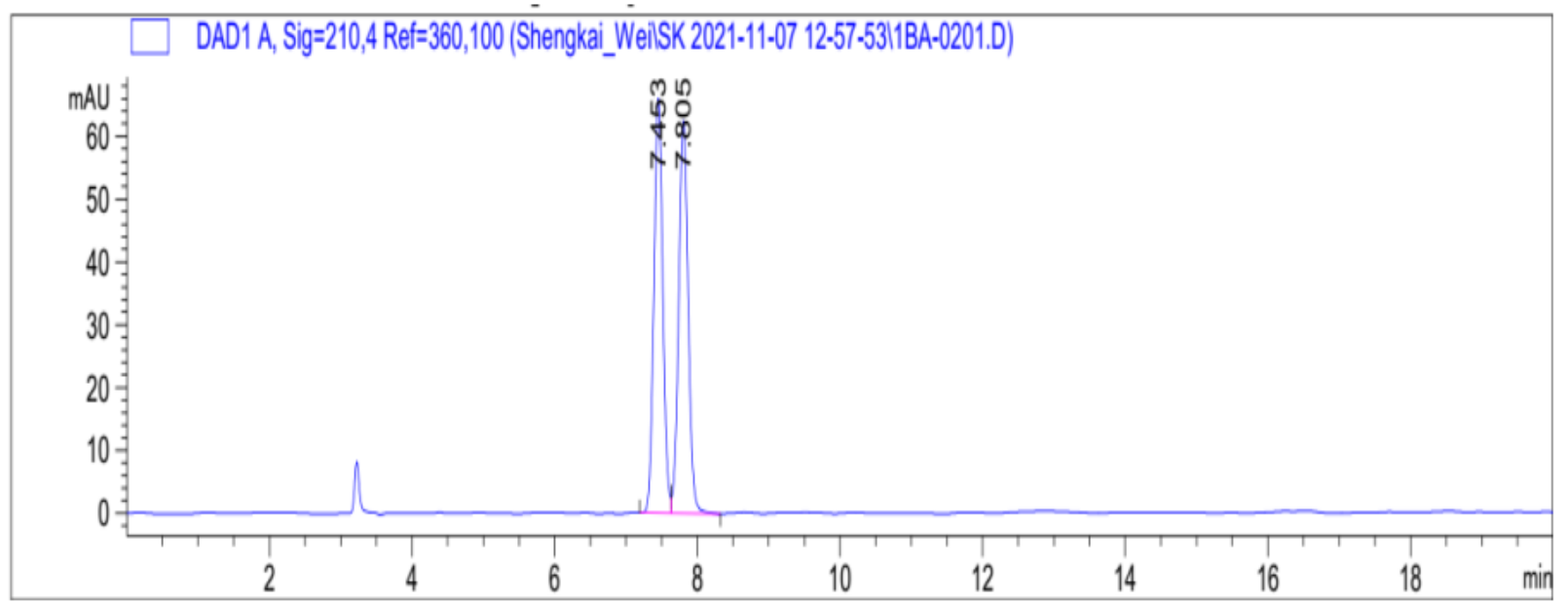

Signal 1: DAD1 A, Sig=210, 4 Ref $=360,100$

\begin{tabular}{|c|c|c|c|c|c|c|}
\hline $\begin{array}{c}\text { Peak } \\
\#\end{array}$ & $\begin{array}{c}\text { RetTime } \\
\text { [min] }\end{array}$ & Type & $\begin{array}{l}\text { Width } \\
\text { [min] }\end{array}$ & $\begin{array}{c}\text { Area } \\
{\left[\mathrm{mAU}^{\star} \mathrm{s}\right]}\end{array}$ & $\begin{array}{l}\text { Height } \\
\text { [mAU] }\end{array}$ & $\begin{array}{c}\text { Area } \\
8\end{array}$ \\
\hline----1 & |------ & & ----- & -------- & -------- & --------1 \\
\hline 1 & 7.453 & BV & 0.1349 & 571.59912 & 66.07492 & 49.6512 \\
\hline 2 & 7.805 & VB & 0.1423 & 579.62927 & 62.45992 & 50.3488 \\
\hline otal & Is : & & & 1151.22839 & 128.53484 & \\
\hline
\end{tabular}




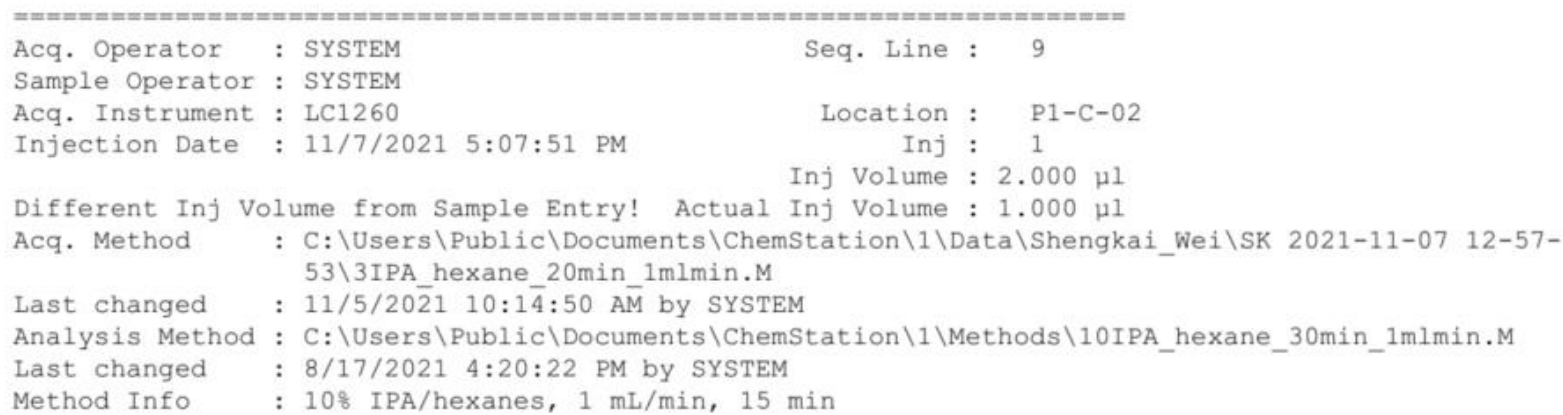<smiles>C[C@H](O)c1ccc(C(F)(F)F)cc1</smiles>

\section{Alcohol derivative of $\mathbf{3 e}$}

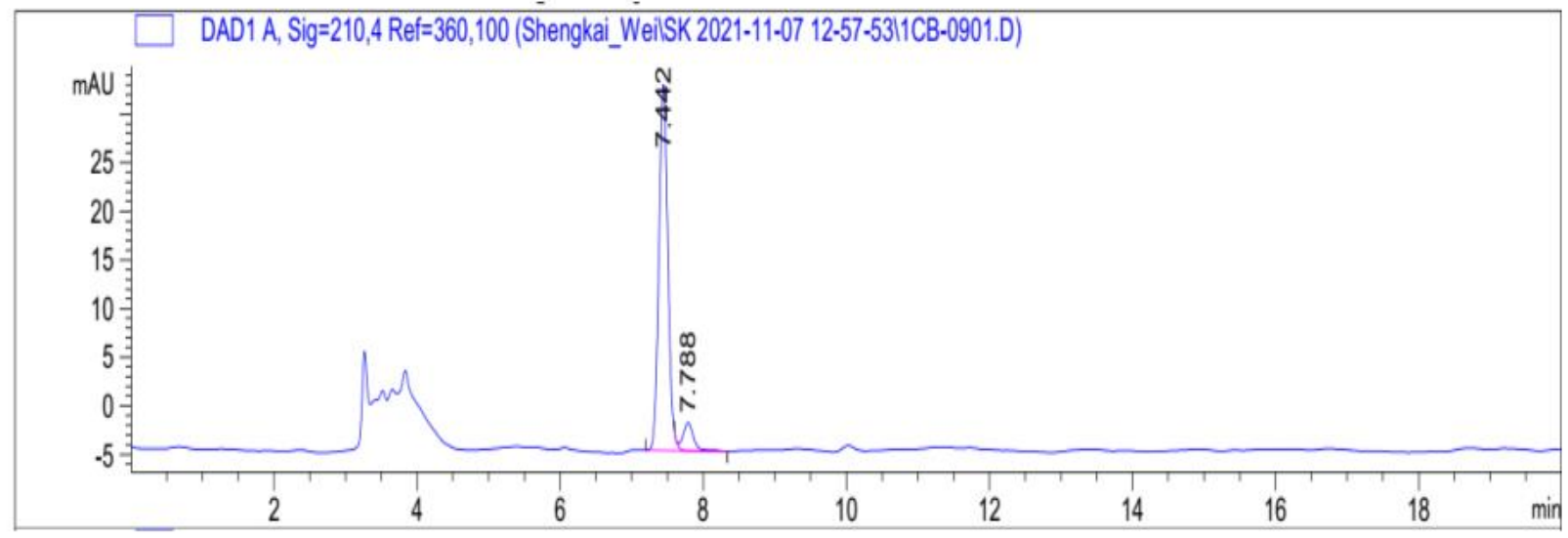

Signal 1: DAD1 A, Sig=210, 4 Ref $=360,100$

\begin{tabular}{|c|c|c|c|c|c|c|}
\hline $\begin{array}{c}\text { Peak } \\
\quad \#\end{array}$ & $\begin{array}{c}\text { RetTime } \\
\text { [min] }\end{array}$ & Type & $\begin{array}{l}\text { Width } \\
\text { [min] }\end{array}$ & $\begin{array}{c}\text { Area } \\
{\left[\mathrm{mAU}{ }^{*} \mathrm{~S}\right]}\end{array}$ & $\begin{array}{l}\text { Height } \\
\text { [mAU] }\end{array}$ & $\begin{array}{c}\text { Area } \\
\frac{8}{8}\end{array}$ \\
\hline-- & $-\cdots--1$ & $|----|$ & $----\infty$ & --------1 & -------1 & -----1 \\
\hline 1 & 7.442 & BV $R$ & 0.1333 & 326.87448 & 37.66133 & 91.5938 \\
\hline 2 & 7.788 & VB $E$ & 0.1582 & 29.99969 & 2.86330 & 8.4062 \\
\hline Total & s: & & & 356.87417 & 40.52463 & \\
\hline
\end{tabular}




\begin{tabular}{|c|c|c|c|}
\hline \multicolumn{2}{|r|}{ SAMPLE } & \multicolumn{2}{|c|}{ INFORMATION } \\
\hline Sample Name: & & Acquired By: & System \\
\hline Sample Type: & Unknown & Sample Set Name & HT_07122021_2 \\
\hline Vial: & 17 & Acq. Method Set: & 3_ŌDH 97_3 1mpm \\
\hline Injection \#: & 1 & Processing Method & Hydroboration \\
\hline Injection Volume: & $10.00 \mathrm{ul}$ & Channel Name: & W2489 ChB \\
\hline Run Time: & 15.0 Minutes & Proc. Chnl. Descr.: & W2489 ChB 220nm \\
\hline Date Acquired: & 7/12/2021 5:22:51 PM CDT & & \\
\hline Date Processed: & 7/13/2021 7:59:32 AM CDT & & \\
\hline
\end{tabular}

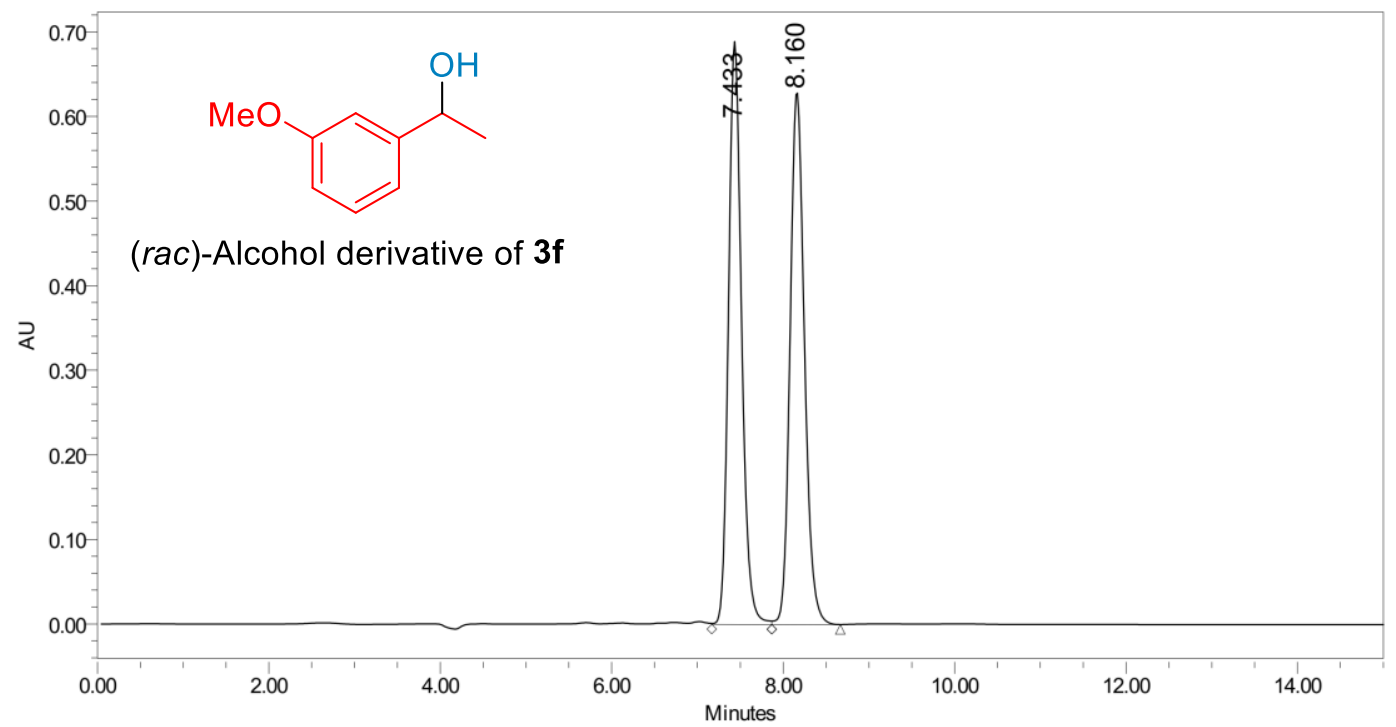

\begin{tabular}{|c|c|c|r|c|}
\hline & RT & Area & $\%$ Area & Height \\
\hline 1 & 7.433 & 7296485 & 49.84 & 688375 \\
\hline 2 & 8.160 & 7344268 & 50.16 & 630328 \\
\hline
\end{tabular}

Reported by User: System

Report Method: Default Individual Report Report Method I[ 1014

Page: 1 of 1
Project Name: Stanley_1\Stanley4 Date Printed:

$7 / 13 / 2021$

8:02:59 AM US/Central 


\begin{tabular}{|c|c|c|c|}
\hline \multicolumn{2}{|r|}{ SAMPLE } & \multicolumn{2}{|c|}{ INFORMATION } \\
\hline Sample Name: & & Acquired By: & System \\
\hline Sample Type: & Unknown & Sample Set Name & HT_07122021_2 \\
\hline Vial: & 16 & Acq. Method Set: & 3_ŌDH 97_3 1mpm \\
\hline Injection \#: & 1 & Processing Method & Hydroboration \\
\hline Injection Volume: & $5.00 \mathrm{ul}$ & Channel Name: & W2489 ChB \\
\hline Run Time: & 15.0 Minutes & Proc. Chnl. Descr.: & W2489 ChB 220nm \\
\hline Date Acquired: & 7/12/2021 5:38:32 PM CDT & & \\
\hline Date Processed: & 11/17/2021 4:36:54 PM CST & & \\
\hline
\end{tabular}

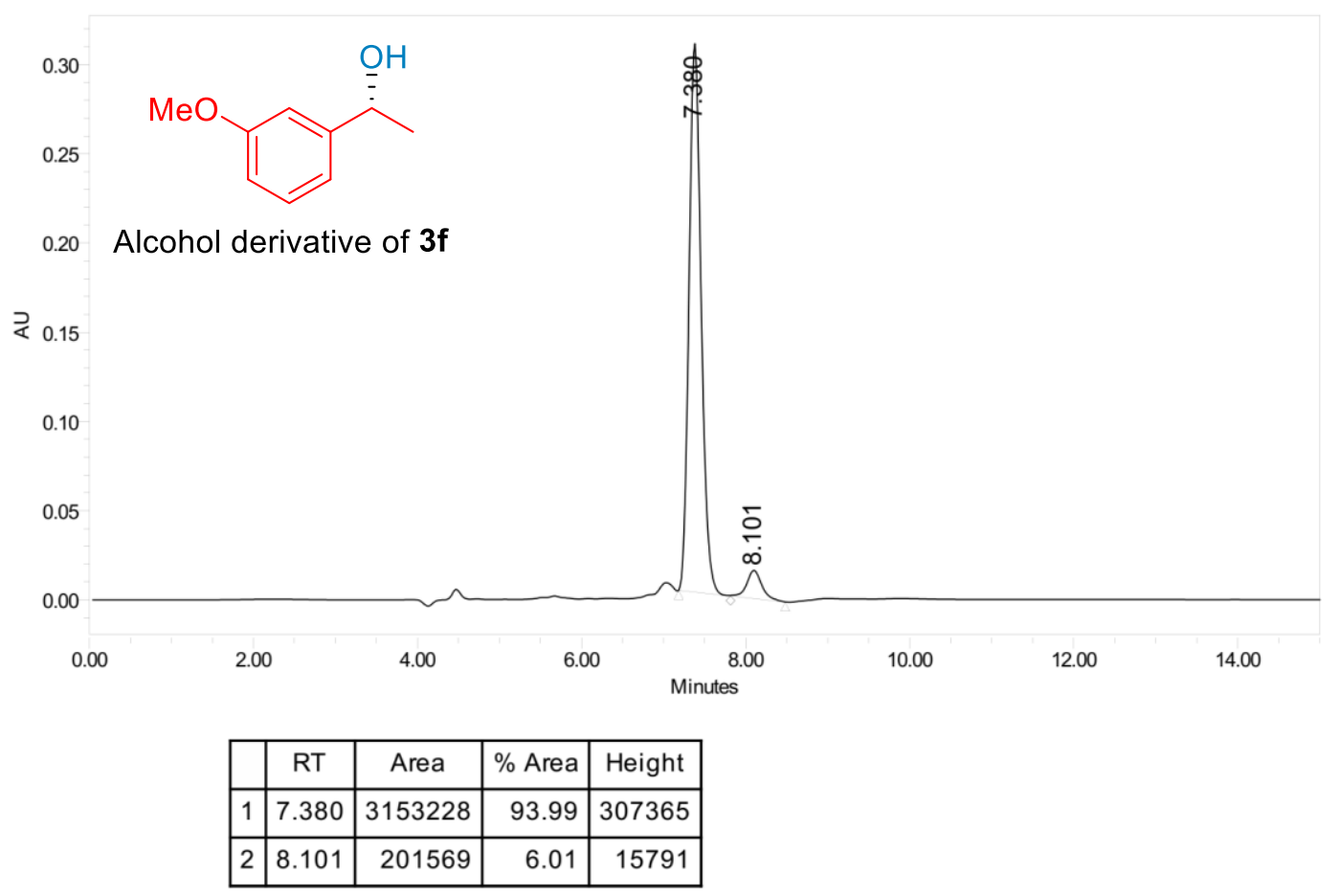

Reported by User: System

Report Method: Default Individual Report Report Method I[ 1014

Page: 1 of 1
Project Name: Stanley_1IStanley4 Date Printed: 11/17/2021 4:39:50 PM US/Central 


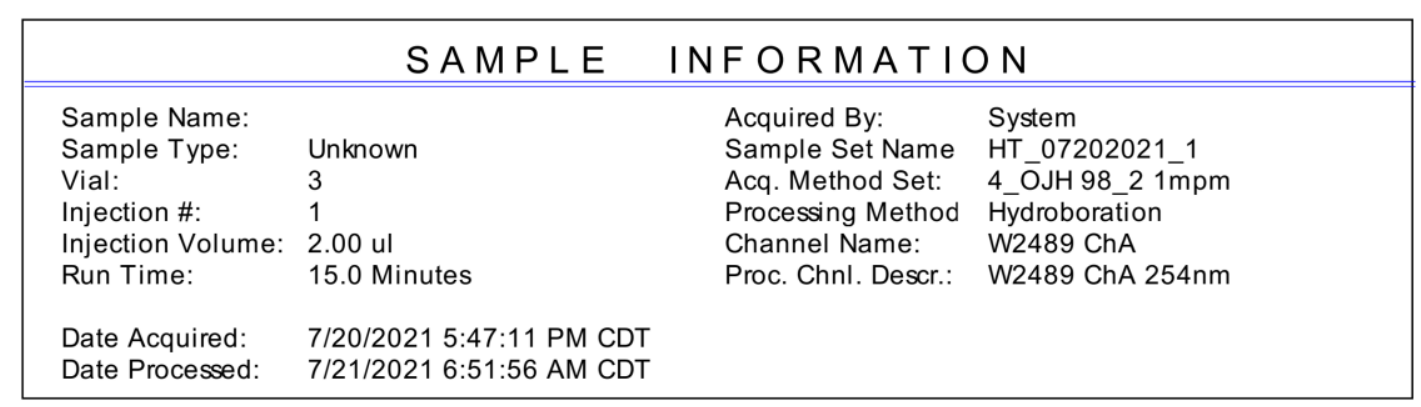

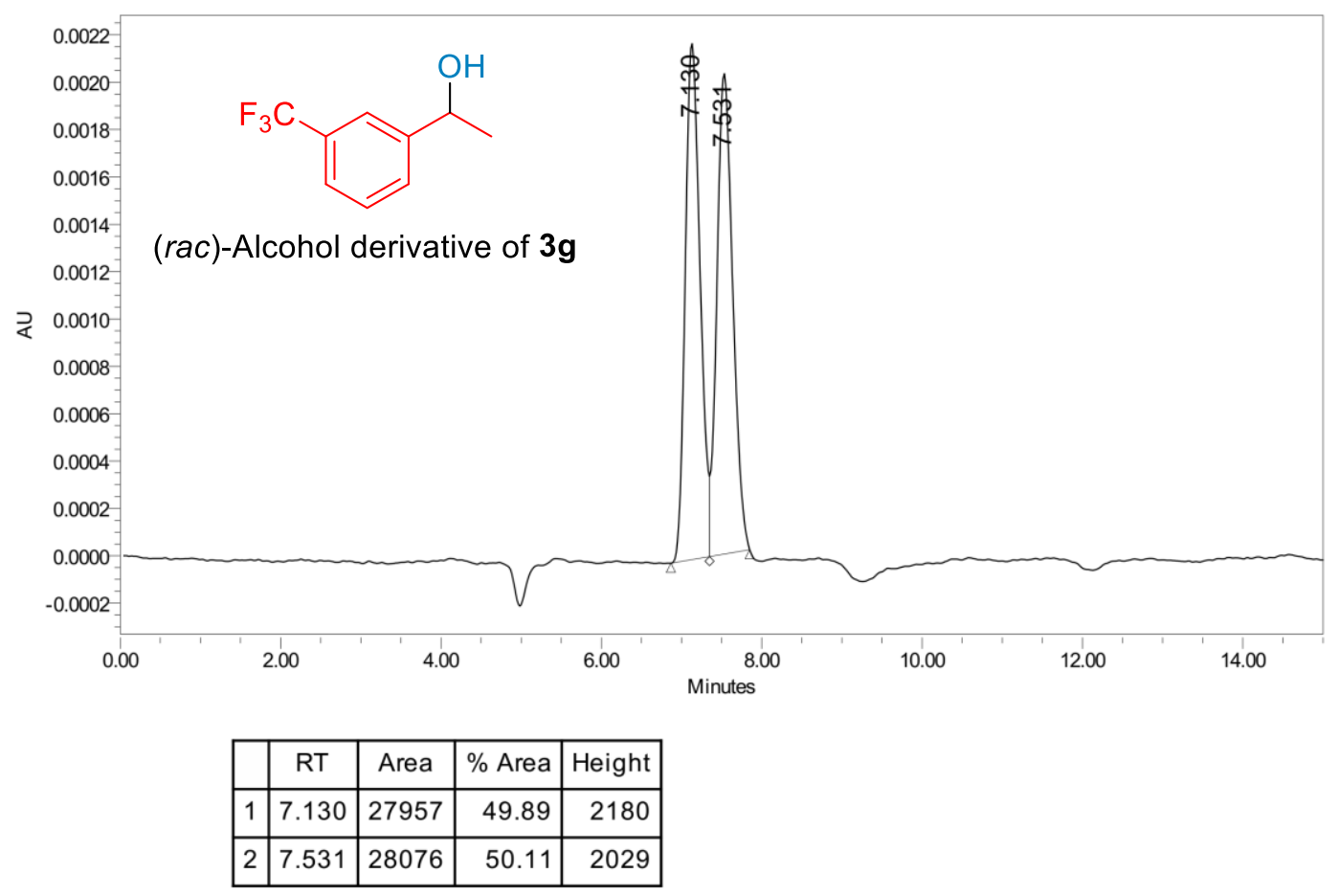

Reported by User: System

Report Method: Default Individual Report Report Method I[ 1014

Page: 1 of 1
Project Name: Stanley_1IStanley4 Date Printed:

$7 / 21 / 2021$

6:56:39 AM US/Central 


\begin{tabular}{|llll|}
\hline & \multicolumn{2}{c|}{ S A M P E } & IN F O R M A T I O N \\
\hline \hline Sample Name: & \multirow{2}{*}{ Unknown } & Acquired By: & System \\
Sample Type: & 2 & Sample Set Name HT_07202021_1 \\
Vial: & 1 & Acq. Method Set: 4_OJH 98_2 1mpm \\
Injection \#: & Processing Method Hydroboration \\
Injection Volume: & $5.00 \mathrm{ul}$ & Channel Name: & W2489 ChB \\
Run Time: & 15.0 Minutes & Proc. Chnl. Descr.: W2489 ChB 220nm \\
& & & \\
Date Acquired: & $7 / 20 / 20215: 00: 12$ PM CDT & \\
Date Processed: & $11 / 29 / 20219: 28: 22$ AM CST & \\
\hline
\end{tabular}

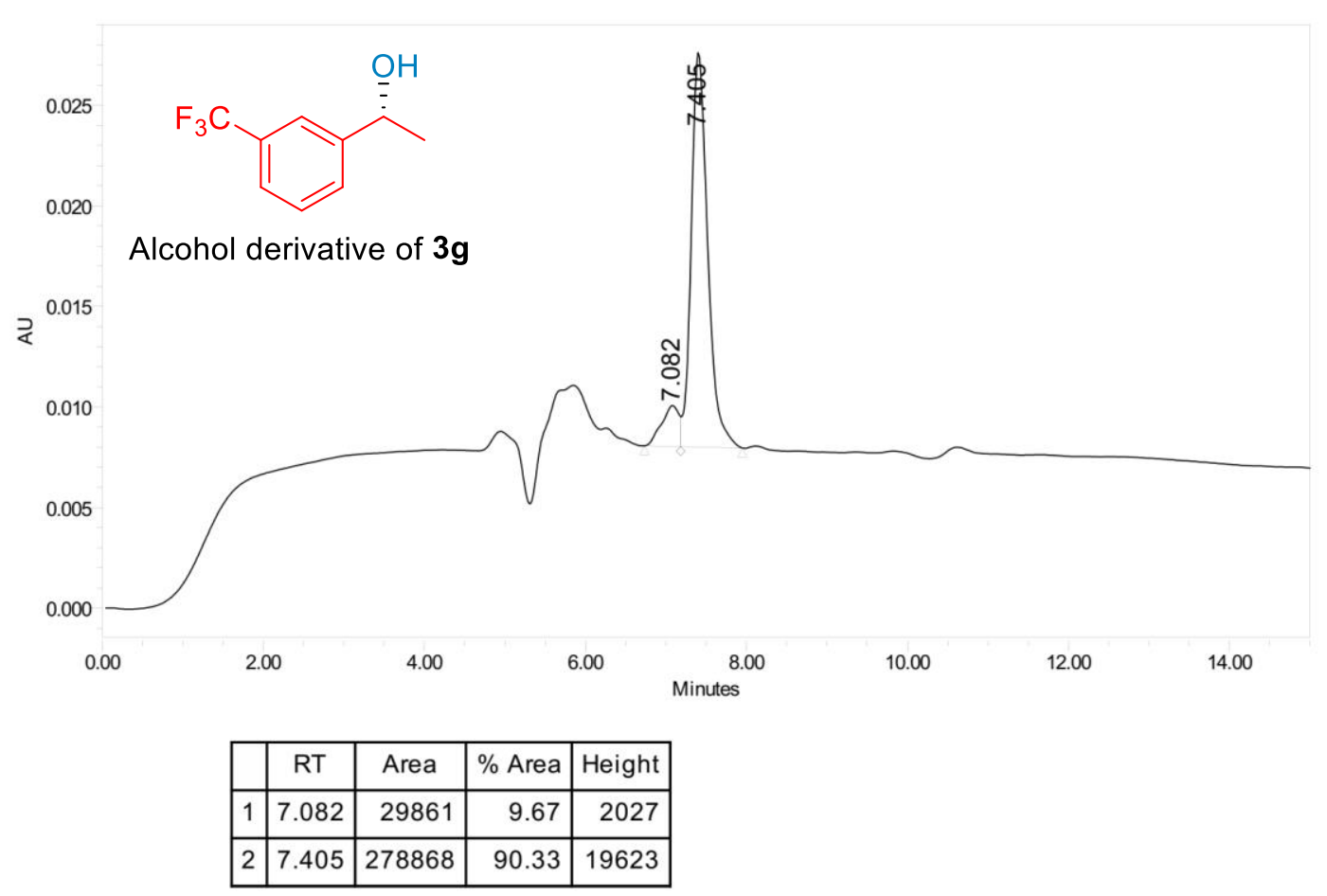

Reported by User: System

Project Name: Stanley_1IStanley4

Report Method: Default Individual Report Date Printed: Report Method I[ 1014 $11 / 29 / 2021$

Page: 1 of 1

9:30:06 AM US/Central 


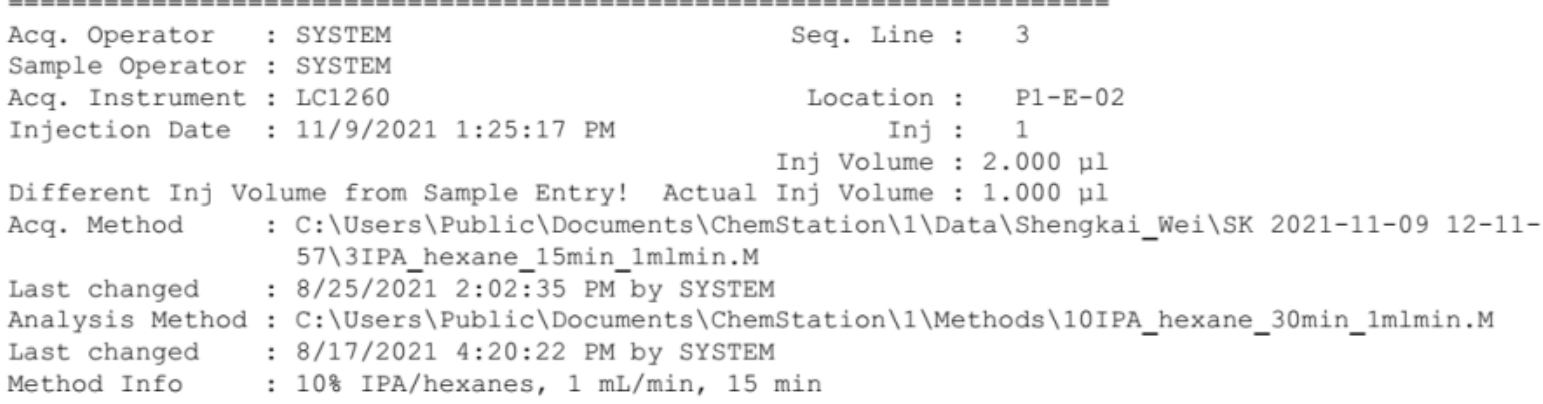<smiles>Cc1ccccc1C(C)O</smiles>

\section{(rac)-Alcohol derivative of $\mathbf{3 h}$}

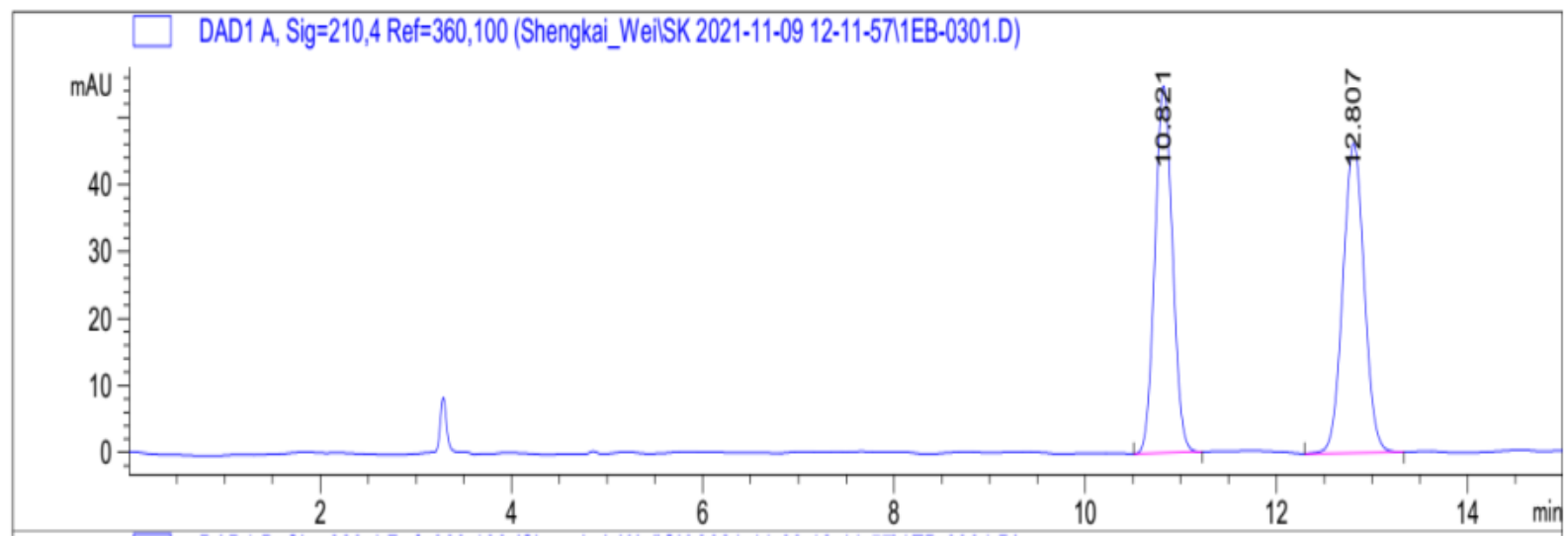

Signal 1: DAD1 A, Sig=210,4 Ref=360,100

\begin{tabular}{|c|c|c|c|c|c|c|}
\hline $\begin{array}{c}\text { Peak } \\
\#\end{array}$ & $\begin{array}{c}\text { RetTime } \\
\text { [min] }\end{array}$ & Type & $\begin{array}{l}\text { Width } \\
\text { [min] }\end{array}$ & $\begin{array}{c}\text { Area } \\
{\left[\mathrm{mAU}{ }^{\star} \mathrm{S}\right]}\end{array}$ & $\begin{array}{l}\text { Height } \\
\text { [mAU] }\end{array}$ & $\begin{array}{c}\text { Area } \\
8\end{array}$ \\
\hline---- & & & ----- & -------- & ------- & ------ \\
\hline 1 & 10.821 & $\mathrm{BB}$ & 0.1985 & 698.10925 & 54.83166 & 49.3804 \\
\hline 2 & 12.807 & $\mathrm{BB}$ & 0.2425 & 715.62921 & 46.26289 & 50.6196 \\
\hline Totá & : & & & 1413.73846 & 09455 & \\
\hline
\end{tabular}




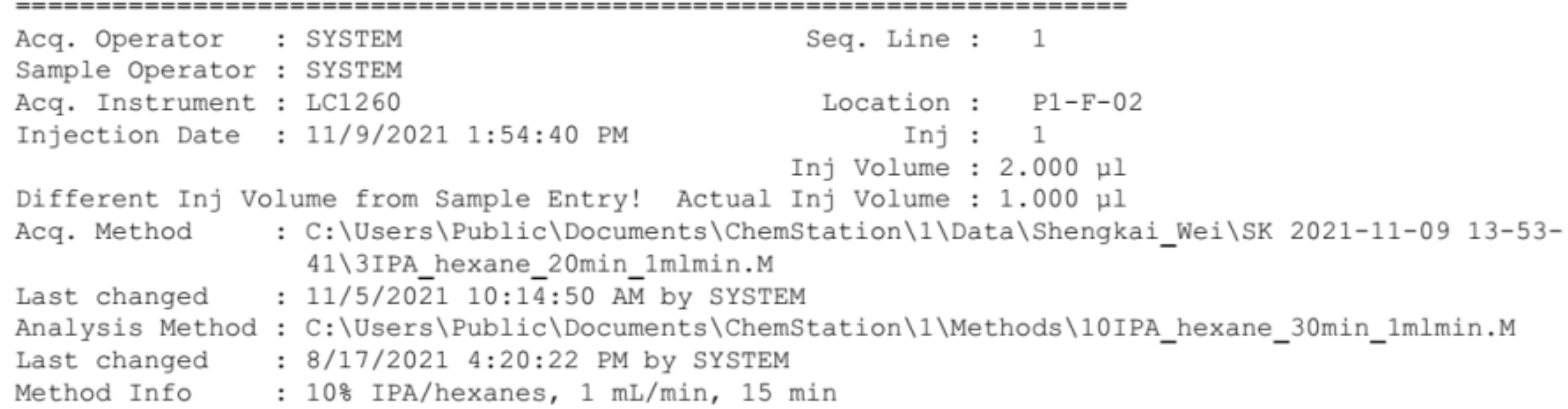<smiles>CCCCc1ccccc1C</smiles>

\section{Alcohol derivative of $\mathbf{3 h}$}

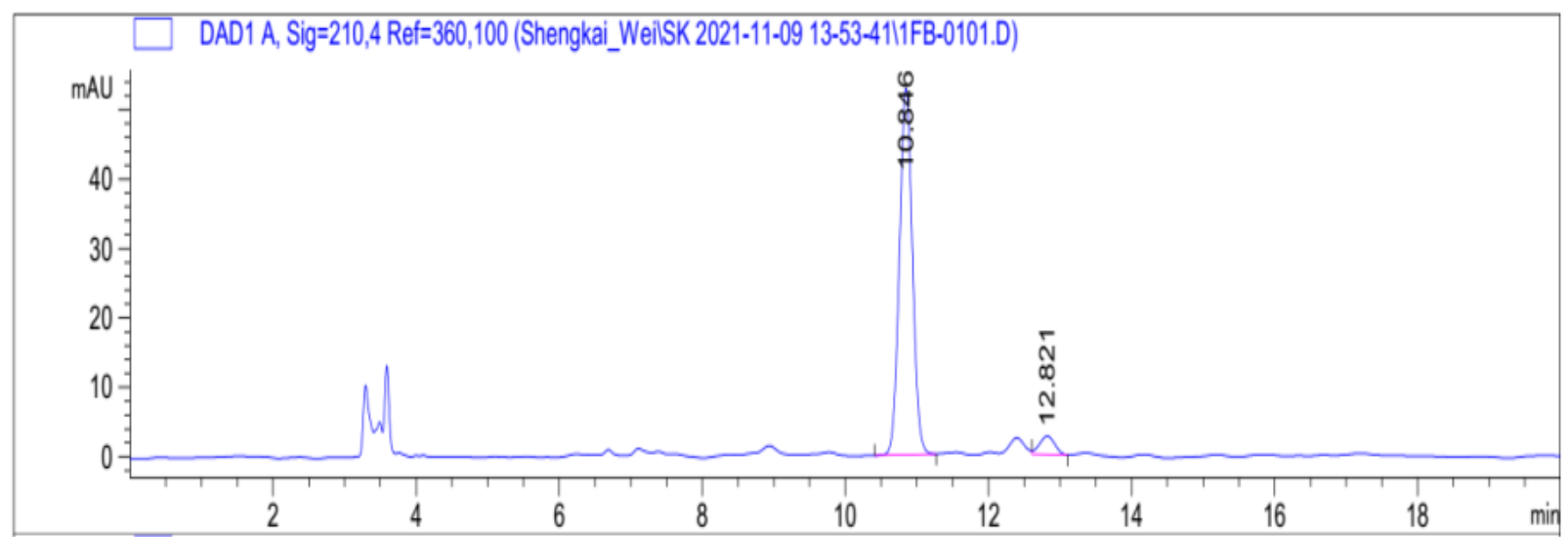

Signal 1: DAD1 A, Sig=210,4 Ref $=360,100$

\begin{tabular}{|c|c|c|c|c|c|c|}
\hline $\begin{array}{c}\text { Peak } \\
\#\end{array}$ & $\begin{array}{c}\text { RetTime } \\
\text { [min] }\end{array}$ & Type & $\begin{array}{l}\text { Width } \\
\text { [min] }\end{array}$ & $\begin{array}{c}\text { Area } \\
{\left[\mathrm{mAU}^{*} \mathrm{~s}\right]}\end{array}$ & $\begin{array}{l}\text { Height } \\
\text { [mAU] }\end{array}$ & $\begin{array}{c}\text { Area } \\
\text { \% }\end{array}$ \\
\hline-- & I----- & & & - - - - - & --n---- & --n-- \\
\hline 1 & 10.846 & $\mathrm{BB}$ & 0.1948 & 664.44879 & 52.80438 & 94.3904 \\
\hline 2 & 12.821 & VB & 0.2305 & 39.48803 & 2.67032 & 5.6096 \\
\hline Total & : & & & 703.93682 & 5.47470 & \\
\hline
\end{tabular}




\begin{tabular}{|c|c|c|c|}
\hline \multicolumn{2}{|r|}{ SAMPLE } & \multicolumn{2}{|c|}{ INFORMATION } \\
\hline Sample Name: & & Acquired By: & System \\
\hline Sample Type: & Unknown & Sample Set Name & HT_07222021 \\
\hline Vial: & 12 & Acq. Method Set: & 2_ĀSH 95_5 1mpm \\
\hline Injection \#: & 1 & Processing Method & Hydroboration \\
\hline Injection Volume: & $10.00 \mathrm{ul}$ & Channel Name: & W2489 ChB \\
\hline Run Time: & 30.0 Minutes & Proc. Chnl. Descr.: & W2489 ChB $220 \mathrm{~nm}$ \\
\hline Date Acquired: & 7/22/2021 9:07:15 AM CDT & & \\
\hline Date Processed: & 11/29/2021 10:58:07 AM CST & & \\
\hline
\end{tabular}

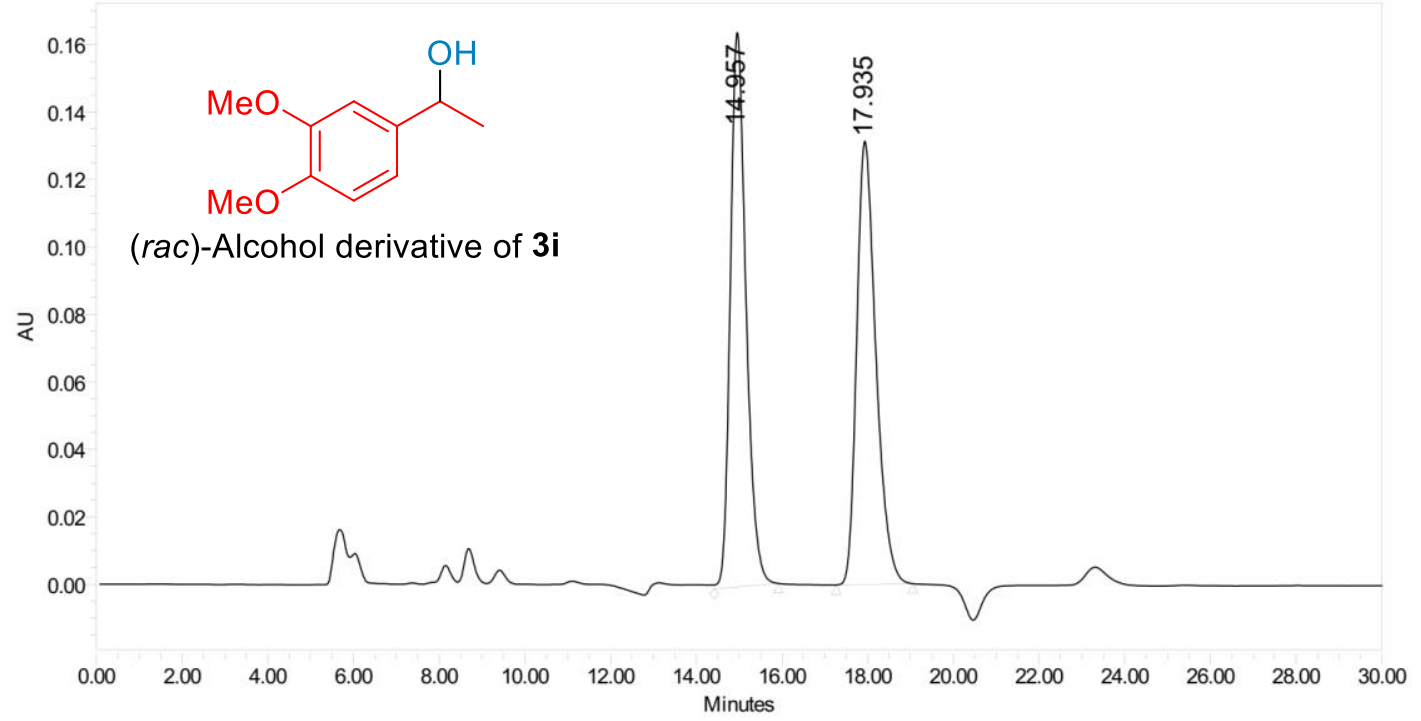

\begin{tabular}{|c|c|c|r|c|}
\hline & RT & Area & $\%$ Area & Height \\
\hline 1 & 14.957 & 4174908 & 50.53 & 164515 \\
\hline 2 & 17.935 & 4086710 & 49.47 & 131441 \\
\hline
\end{tabular}

Reported by User: System

Report Method: Default Individual Report Report Method I[ 1014

Page: 1 of 1
Project Name: Stanley_1\Stanley4 Date Printed: $11 / 29 / 2021$ 10:59:11 AM US/Centra 


\begin{tabular}{|c|c|c|c|}
\hline \multicolumn{2}{|r|}{ SAMPLE } & \multicolumn{2}{|c|}{ INFORMATION } \\
\hline Sample Name: & & Acquired By: & System \\
\hline Sample Type: & Unknown & Sample Set Name & HT_07212021 \\
\hline Vial: & 13 & Acq. Method Set: & 2_ĀSH 95_5 1mpm \\
\hline Injection \#: & 1 & Processing Method & Hydroboration \\
\hline Injection Volume: & 20.00 ul & Channel Name: & W2489 ChB \\
\hline Run Time: & 30.0 Minutes & Proc. Chnl. Descr.: & W2489 ChB $220 \mathrm{~nm}$ \\
\hline Date Acquired: & 7/21/2021 8:06:15 PM CDT & & \\
\hline Date Processed: & 11/29/2021 11:00:29 AM CST & & \\
\hline
\end{tabular}

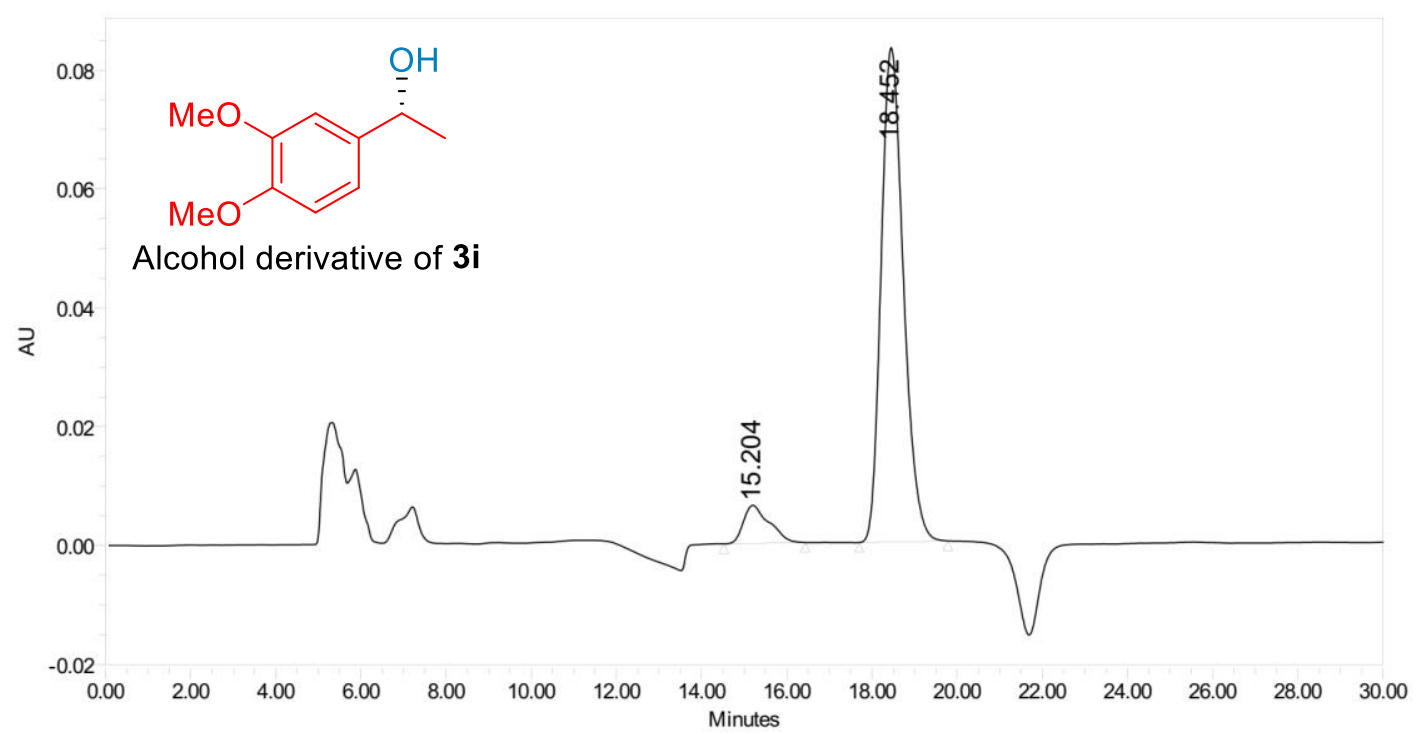

\begin{tabular}{|c|c|r|r|r|}
\hline & RT & Area & $\%$ Area & Height \\
\hline 1 & 15.204 & 268690 & 8.16 & 6401 \\
\hline 2 & 18.452 & 3022959 & 91.84 & 83190 \\
\hline
\end{tabular}

Reported by User: System

Report Method: Default Individual Report Report Method I[ 1014

Page: 1 of 1
Project Name: Stanley_1\Stanley4 Date Printed: $11 / 29 / 2021$ 11:01:04 AM US/Centra 


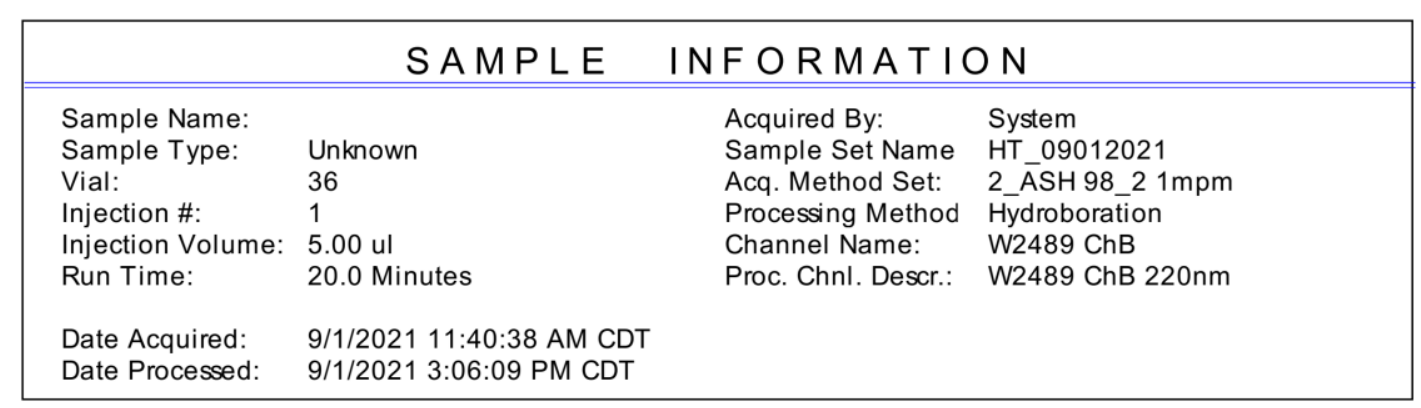

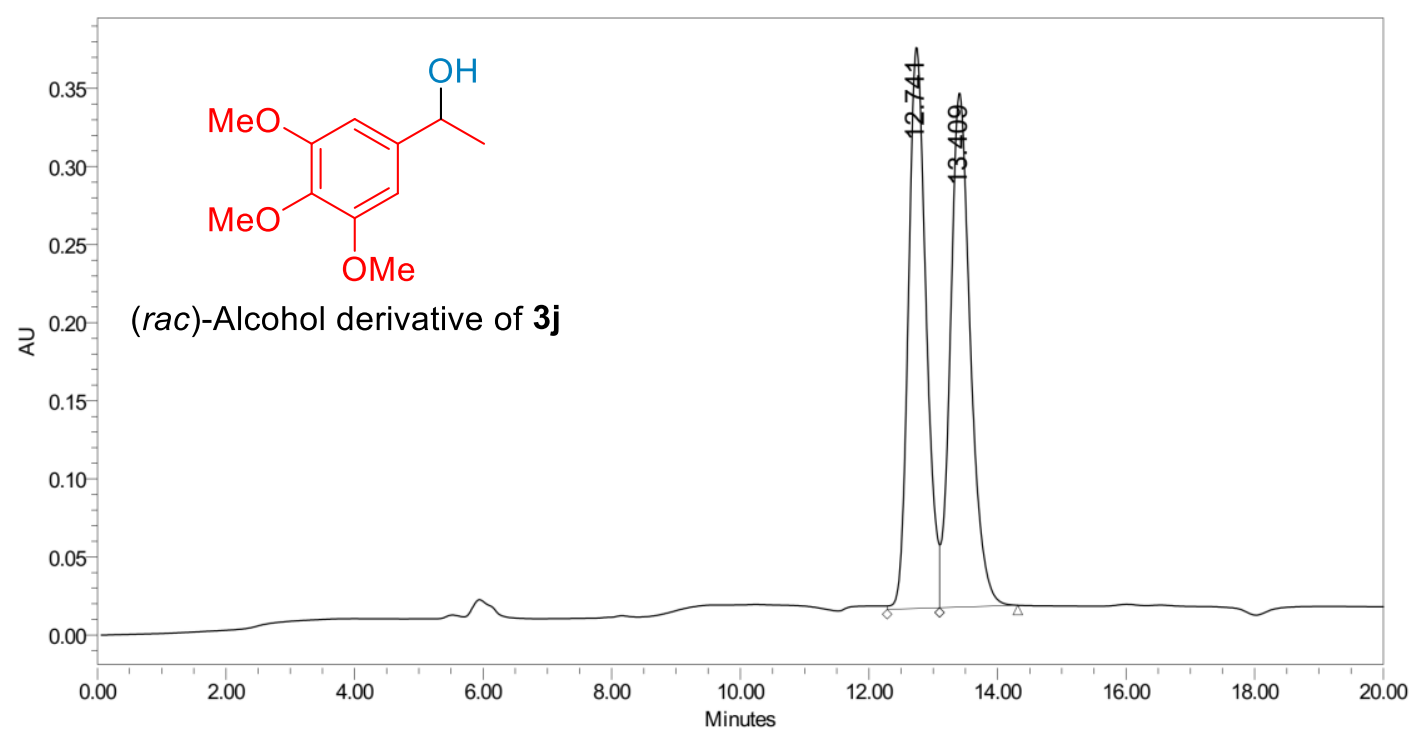

\begin{tabular}{|c|c|c|r|c|}
\hline & RT & Area & $\%$ Area & Height \\
\hline 1 & 12.741 & 6906364 & 49.18 & 359941 \\
\hline 2 & 13.409 & 7136662 & 50.82 & 329559 \\
\hline
\end{tabular}

Reported by User: System

Report Method: Default Individual Report Report Method I[ 1014

Page: 1 of 1
Project Name: Stanley_1\Stanley4

Date Printed:

$9 / 1 / 2021$

3:08:48 PM US/Central 


\begin{tabular}{|llll|}
\hline & \multicolumn{2}{c|}{ S A M P E } & IN F O R M A T I O N \\
\hline \hline Sample Name: & & Acquired By: & System \\
Sample Type: & Unknown & Sample Set Name & HT_07172021 \\
Vial: & 5 & Acq. Method Set: & 2_ASH 98_2 1mpm \\
Injection \#: & 1 & Processing Method Hydroboration \\
Injection Volume: & $2.00 \mathrm{ul}$ & Channel Name: & W2489 ChB \\
Run Time: & 20.0 Minutes & Proc. Chnl. Descr.: W2489 ChB 220nm \\
& & & \\
Date Acquired: & $7 / 17 / 2021$ 12:50:59 PM CDT & & \\
Date Processed: & $11 / 4 / 2021$ & & \\
\hline
\end{tabular}

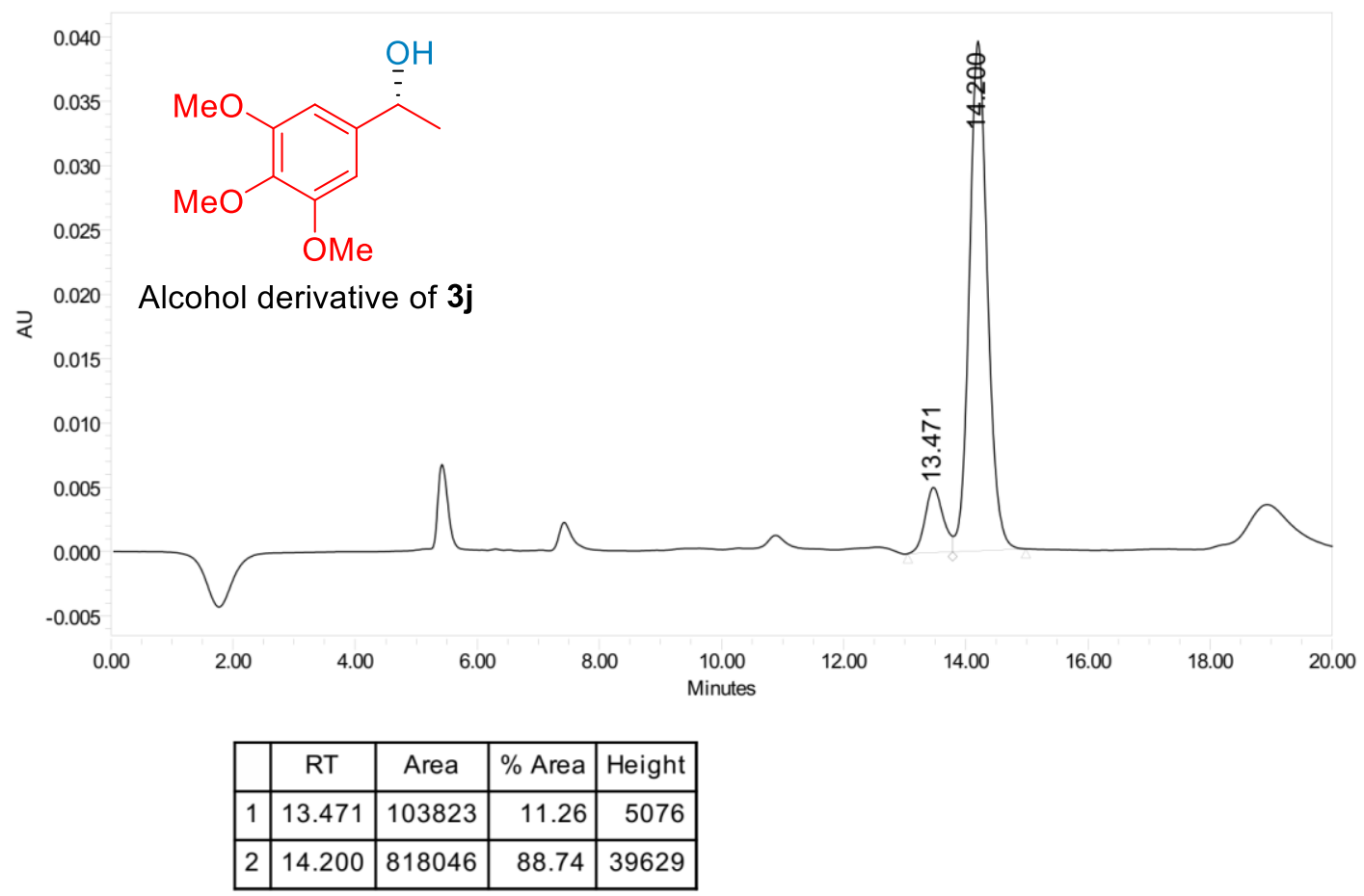

Reported by User: System

Report Method: Default Individual Report Report Method I[ 1014

Page: 1 of 1
Project Name: Stanley_1\Stanley4 Date Printed:

$11 / 4 / 2021$

1:57:45 PM US/Central 


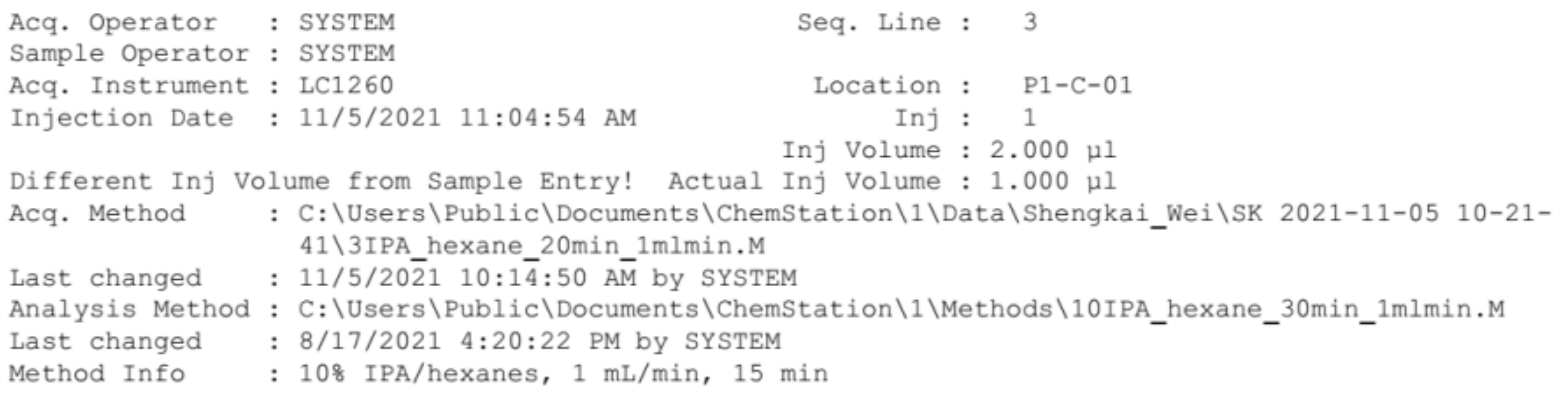<smiles>CC(O)/C=C/c1ccccc1</smiles>

(rac)-Alcohol derivative of $\mathbf{3 k}$

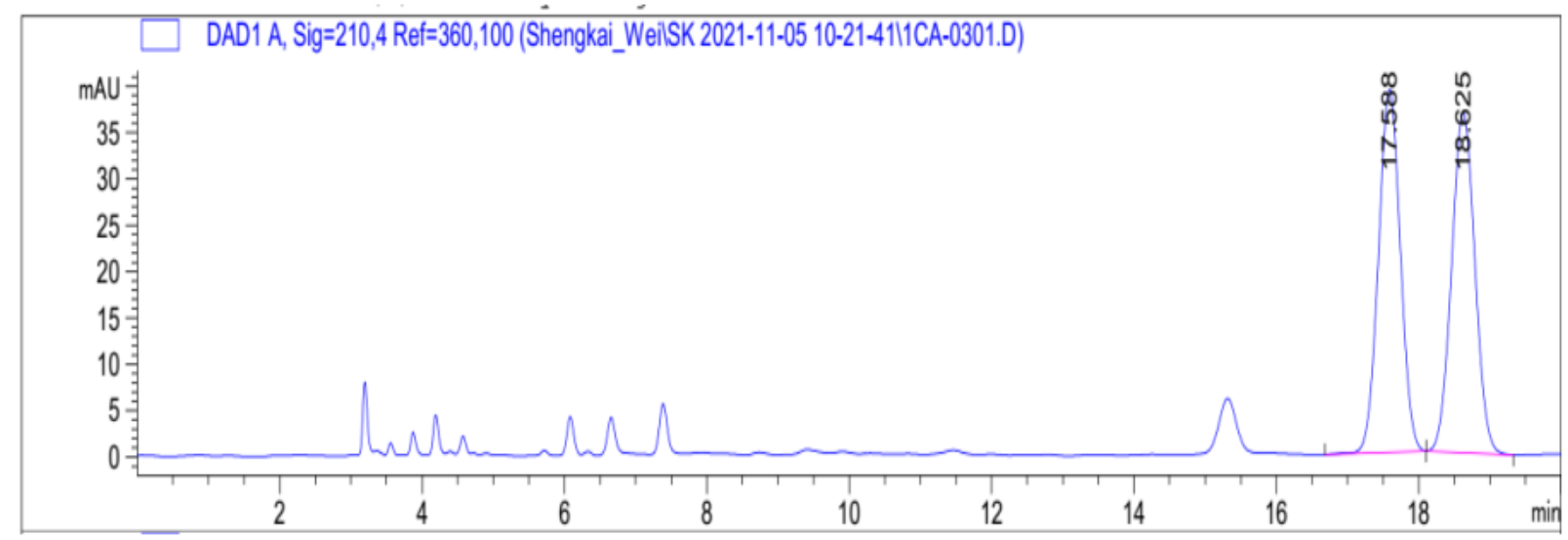

Signal 1: DAD1 A, Sig=210,4 Ref=360,100

\begin{tabular}{|c|c|c|c|c|c|c|}
\hline $\begin{array}{c}\text { Peak } \\
\#\end{array}$ & $\begin{array}{c}\text { RetTime } \\
\text { [min] }\end{array}$ & Type & $\begin{array}{l}\text { Width } \\
\text { [min] }\end{array}$ & $\begin{array}{c}\text { Area } \\
{\left[\mathrm{mAU}^{\star} \mathrm{s}\right]}\end{array}$ & $\begin{array}{l}\text { Height } \\
\text { [mAU] }\end{array}$ & $\begin{array}{c}\text { Area } \\
\text { of }\end{array}$ \\
\hline \multicolumn{7}{|c|}{ 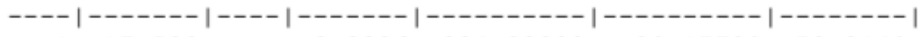 } \\
\hline 1 & 17.588 & BB & 0.3326 & 834.80988 & 39.17729 & 50.0149 \\
\hline 2 & 18.625 & $\mathrm{BB}$ & 0.3571 & 834.31146 & 36.71002 & 49.9851 \\
\hline tal & Ls : & & & 1669.12134 & 88731 & \\
\hline
\end{tabular}




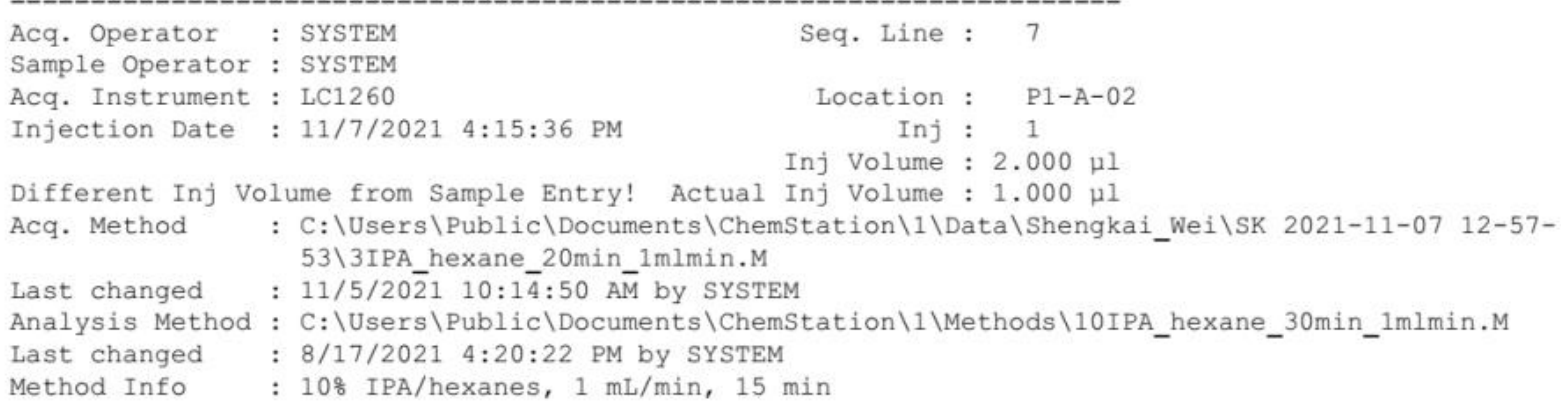<smiles>C[C@@H](O)/C=C/c1ccccc1</smiles>

\section{Alcohol derivative of $\mathbf{3 k}$}

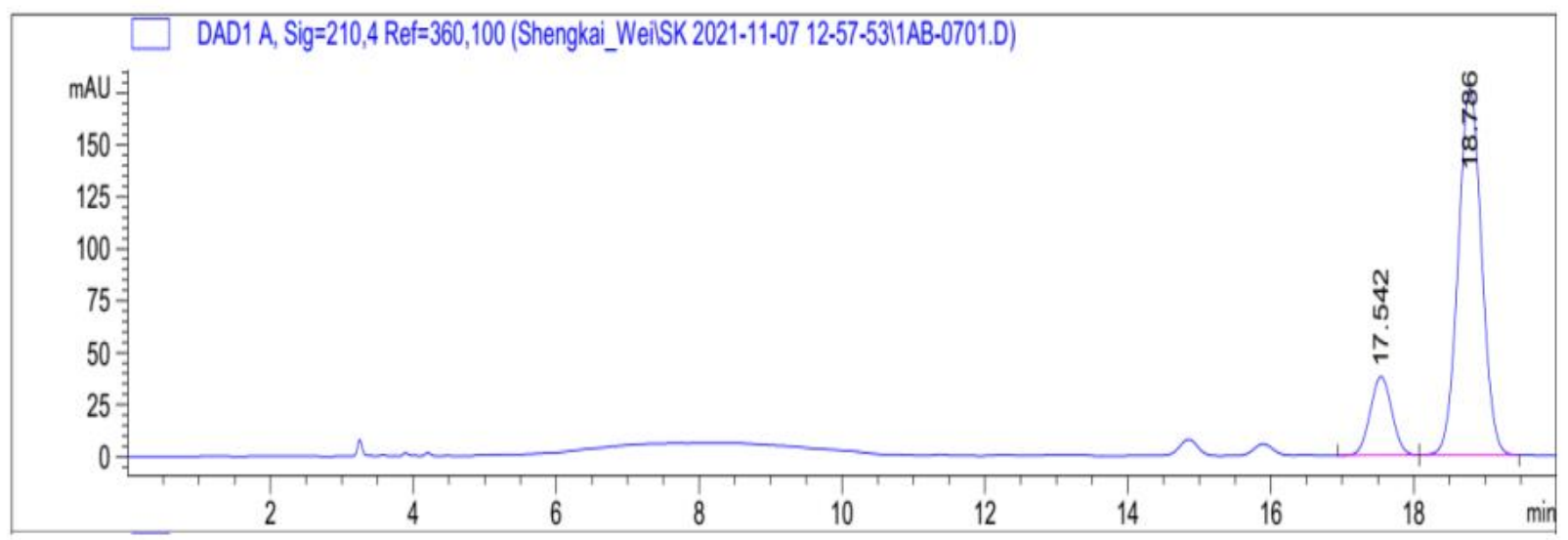

Signal 1: DAD1 A, Sig=210, 4 Ref $=360,100$

\begin{tabular}{|c|c|c|c|c|c|c|}
\hline $\begin{array}{c}\text { Peak } \\
\#\end{array}$ & $\begin{array}{c}\text { RetTime } \\
\text { [min] }\end{array}$ & Type & $\begin{array}{l}\text { Width } \\
\text { [min] }\end{array}$ & $\begin{array}{c}\text { Area } \\
{\left[m A U^{\star} s\right]}\end{array}$ & $\begin{array}{l}\text { Height } \\
\text { [mAU] }\end{array}$ & $\begin{array}{c}\text { Area } \\
\frac{8}{8}\end{array}$ \\
\hline \multicolumn{7}{|c|}{ 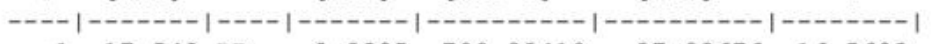 } \\
\hline 1 & 17.542 & $\mathrm{BB}$ & 0.3305 & 799.23419 & 37.82676 & 16.5602 \\
\hline 2 & 18.786 & BB & 0.3557 & 4027.01245 & 176.82835 & 83.4398 \\
\hline Tot & : & & & 4826.24664 & 65511 & \\
\hline
\end{tabular}




\begin{tabular}{|llll|}
\hline & \multicolumn{2}{c|}{ S A M P E } & IN F O R M A T I O N \\
\hline \hline Sample Name: & & Acquired By: & System \\
Sample Type: & Unknown & Sample Set Name & HT_09052021 \\
Vial: & 33 & Acq. Method Set: 4_OJH 90_10 1mpm \\
Injection \#: & 1 & Processing Method Hydroboration \\
Injection Volume: & 10.00 ul & Channel Name: & W2489 ChB \\
Run Time: & 25.0 Minutes & Proc. Chnl. Descr.: W2489 ChB 220nm \\
& & & \\
Date Acquired: & $9 / 5 / 2021$ 1:37:33 PM CDT & & \\
Date Processed: & $9 / 5 / 20213: 02: 02$ PM CDT & & \\
\hline
\end{tabular}

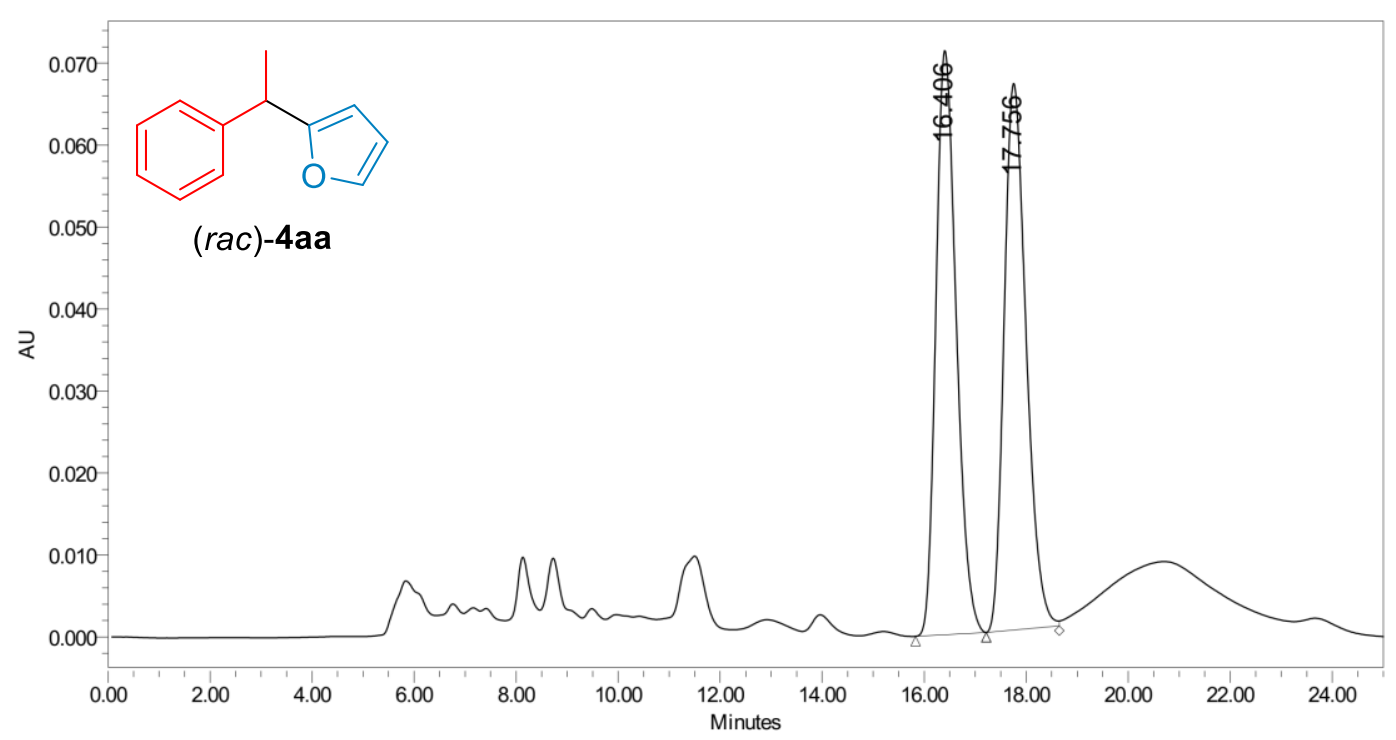

\begin{tabular}{|c|c|c|r|c|}
\hline & RT & Area & $\%$ Area & Height \\
\hline 1 & 16.406 & 1975314 & 49.64 & 71312 \\
\hline 2 & 17.756 & 2003618 & 50.36 & 66715 \\
\hline
\end{tabular}

Reported by User: System

Report Method: Default Individual Report Report Method I[ 1014

Page: 1 of 1
Project Name: Stanley_1\Stanley4

Date Printed:

$9 / 5 / 2021$

3:02:29 PM US/Central 


\begin{tabular}{|llll|}
\hline & \multicolumn{2}{c|}{ S A M P E } & IN F O R M A T I O N \\
\hline \hline Sample Name: & Unknown & Acquired By: & System \\
Sample Type: & 32 & Sample Set Name HT_09052021 \\
Vial: & 1 & Acq. Method Set: 4_OJH 95_5 1mpm \\
Injection \#: & Processing Method Hydroboration \\
Injection Volume: & $10.00 \mathrm{ul}$ & Channel Name: & W2489 ChB \\
Run Time: & 25.0 Minutes & Proc. Chnl. Descr.: W2489 ChB 220nm \\
& & & \\
Date Acquired: & $9 / 5 / 20212: 03: 26$ PM CDT & & \\
Date Processed: & $9 / 5 / 20213: 01: 22$ PM CDT & \\
\hline
\end{tabular}

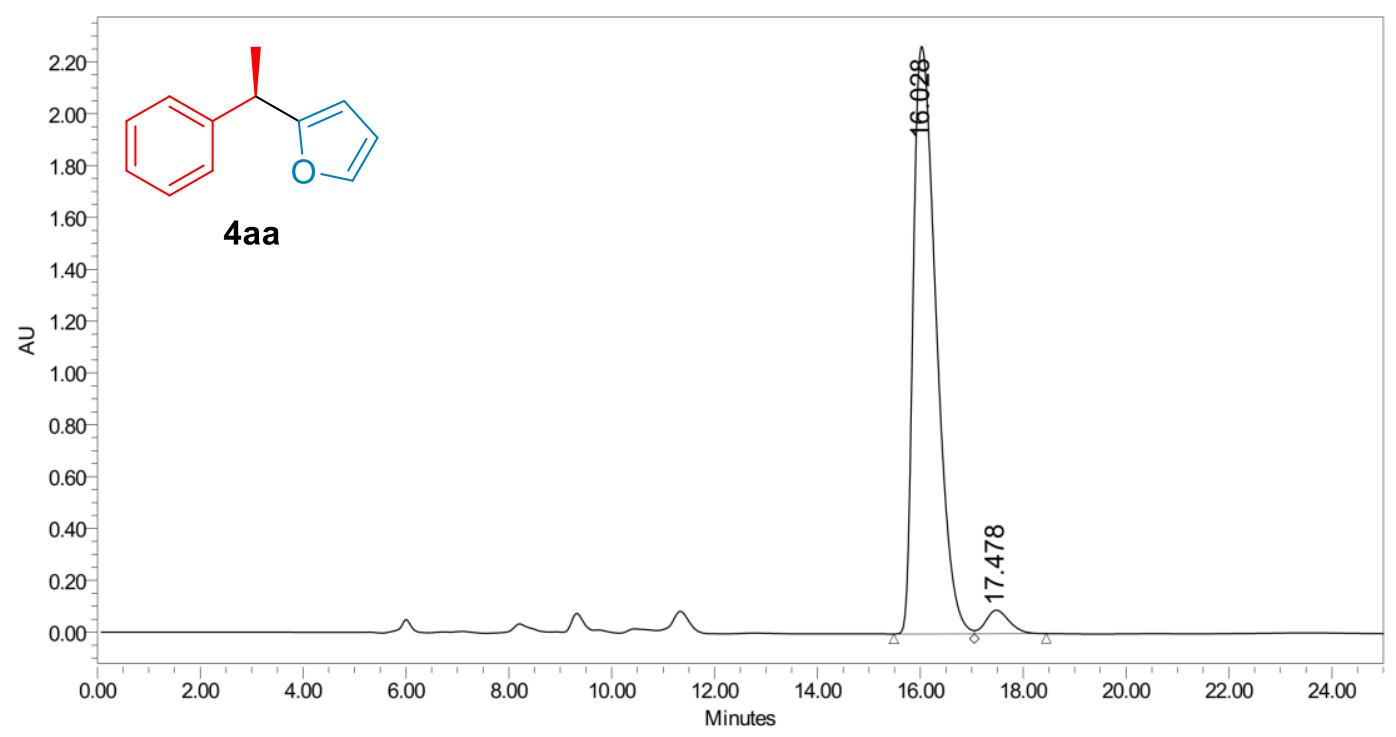

\begin{tabular}{|l|c|c|r|r|}
\hline & RT & Area & $\%$ Area & Height \\
\hline 1 & 16.028 & 70131264 & 96.09 & 2266702 \\
\hline 2 & 17.478 & 2854628 & 3.91 & 90891 \\
\hline
\end{tabular}

Reported by User: System

Report Method: Default Individual Report Report Method I[ 1014

Page: 1 of 1
Project Name: Stanley_1\Stanley4

Date Printed:

$9 / 5 / 2021$

3:03:04 PM US/Central 


\begin{tabular}{|llll|}
\hline & \multicolumn{2}{c|}{ S A M P E } & IN F O R M A T I O N \\
\hline \hline Sample Name: & & Acquired By: & System \\
Sample Type: & Unknown & Sample Set Name & HT_09022021_4 \\
Vial: & 34 & Acq. Method Set: 4_OJH 90_10 1mpm \\
Injection \#: & 1 & Processing Method Hydroboration \\
Injection Volume: & 10.00 ul & Channel Name: & W2489 ChB \\
Run Time: & 30.0 Minutes & Proc. Chnl. Descr.: W2489 ChB 220nm \\
& & & \\
Date Acquired: & $9 / 2 / 20216: 09: 52$ PM CDT & & \\
Date Processed: & $9 / 3 / 20219: 57: 48$ AM CDT & \\
\hline
\end{tabular}

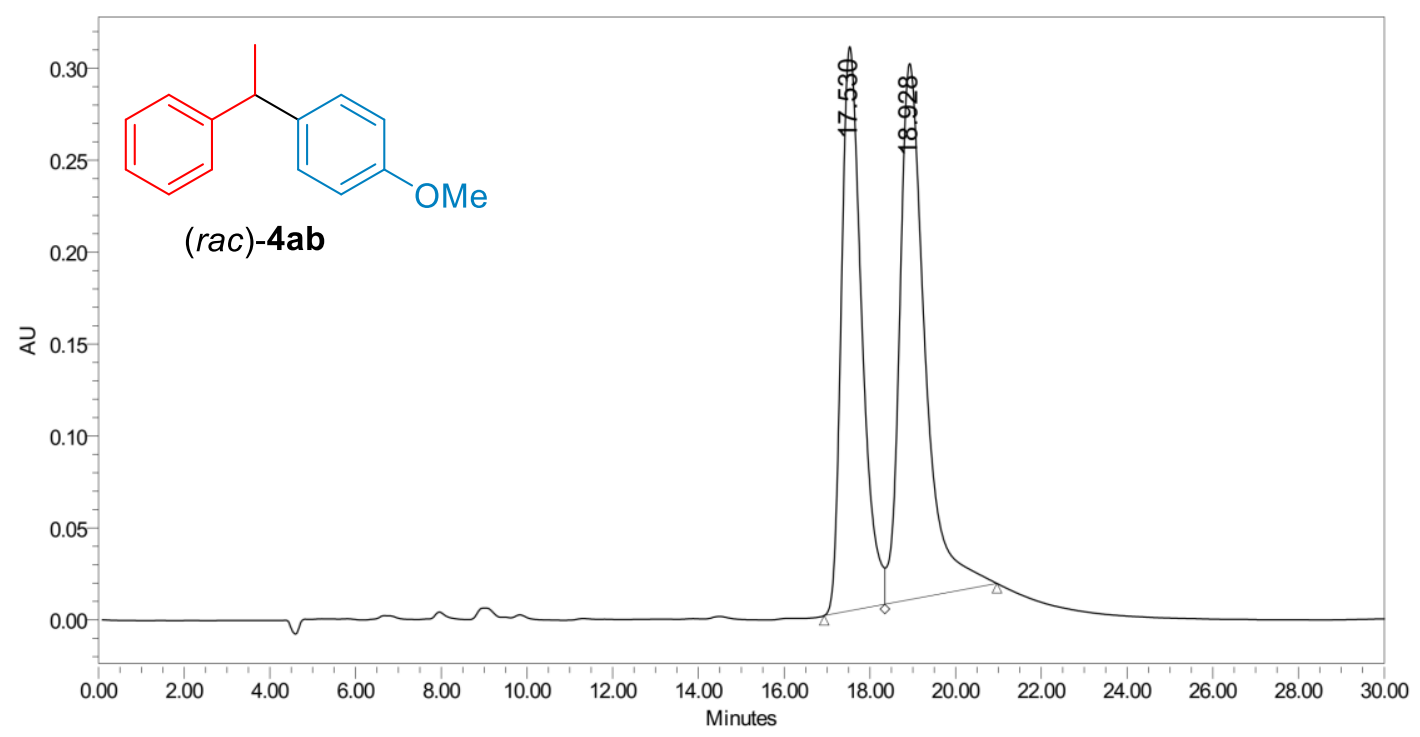

\begin{tabular}{|c|c|c|r|c|}
\hline & RT & Area & \% Area & Height \\
\hline 1 & 17.530 & 10128814 & 45.73 & 306943 \\
\hline 2 & 18.928 & 12021397 & 54.27 & 291653 \\
\hline
\end{tabular}

Reported by User: System

Report Method: Default Individual Report Report Method I[ 1014

Page: 1 of 1
Project Name: Stanley_1\Stanley4 Date Printed: $9 / 3 / 2021$ 9:59:01 AM US/Central 


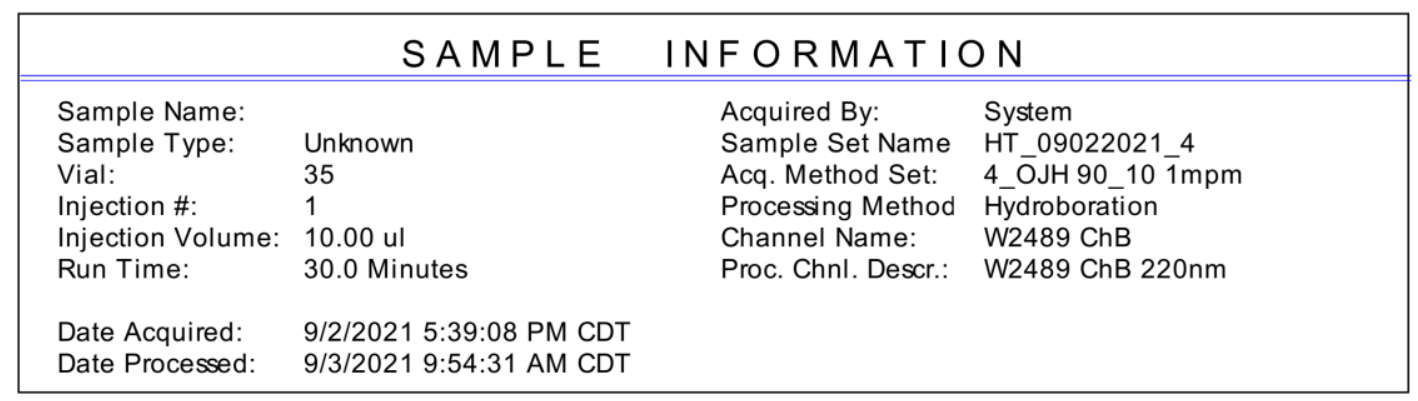

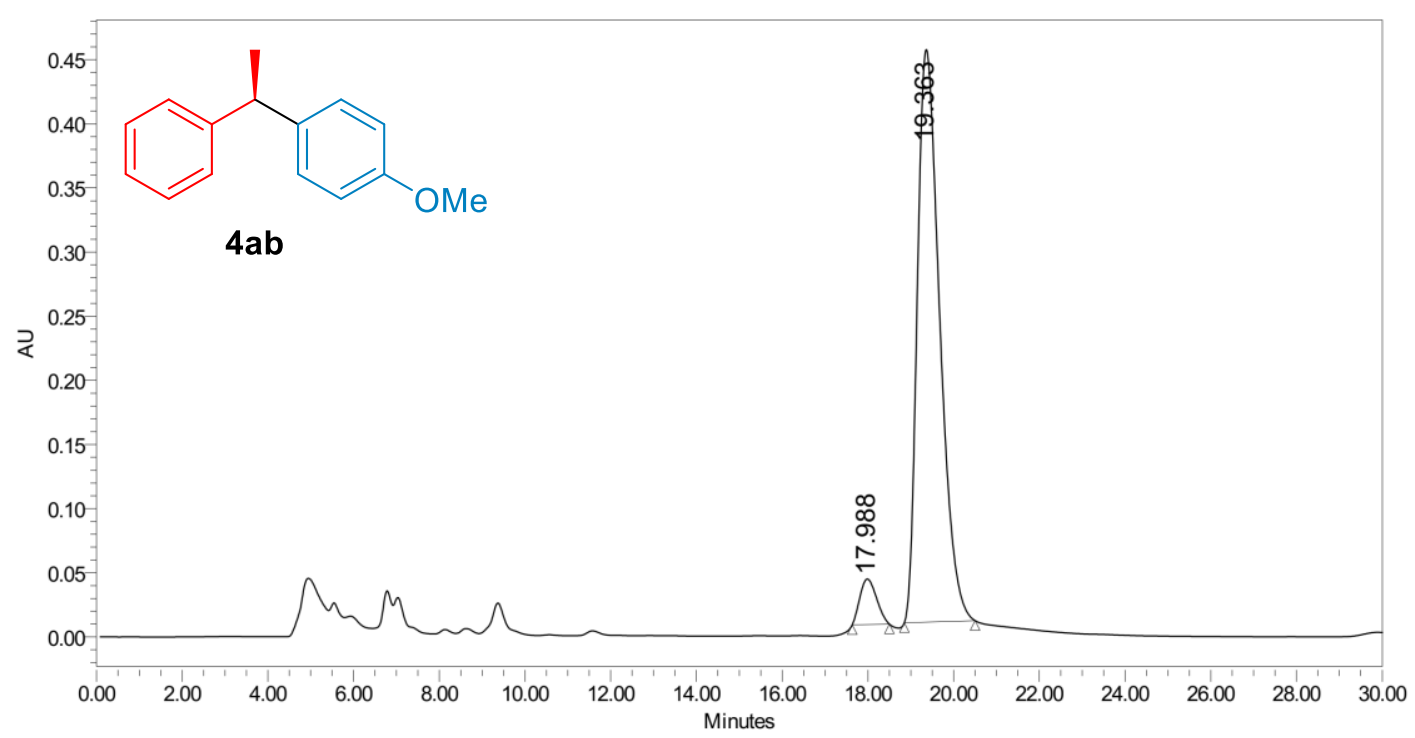

\begin{tabular}{|c|c|r|r|r|}
\hline & RT & Area & $\%$ Area & Height \\
\hline 1 & 17.988 & 951282 & 5.56 & 35412 \\
\hline 2 & 19.363 & 16172030 & 94.44 & 446342 \\
\hline
\end{tabular}

Reported by User: System

Report Method: Default Individual Report Report Method I[ 1014

Page: 1 of 1
Project Name: Stanley_1\Stanley4 Date Printed: $9 / 3 / 2021$

9:58:32 AM US/Central 
10. ${ }^{1} \mathrm{H},{ }^{13} \mathrm{C},{ }^{19} \mathrm{~F}$ and ${ }^{11} \mathrm{~B}$ NMR Spectra for Compounds 


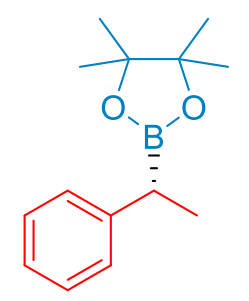

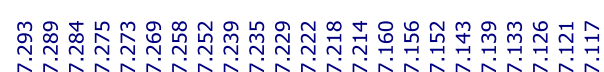

$3 a$

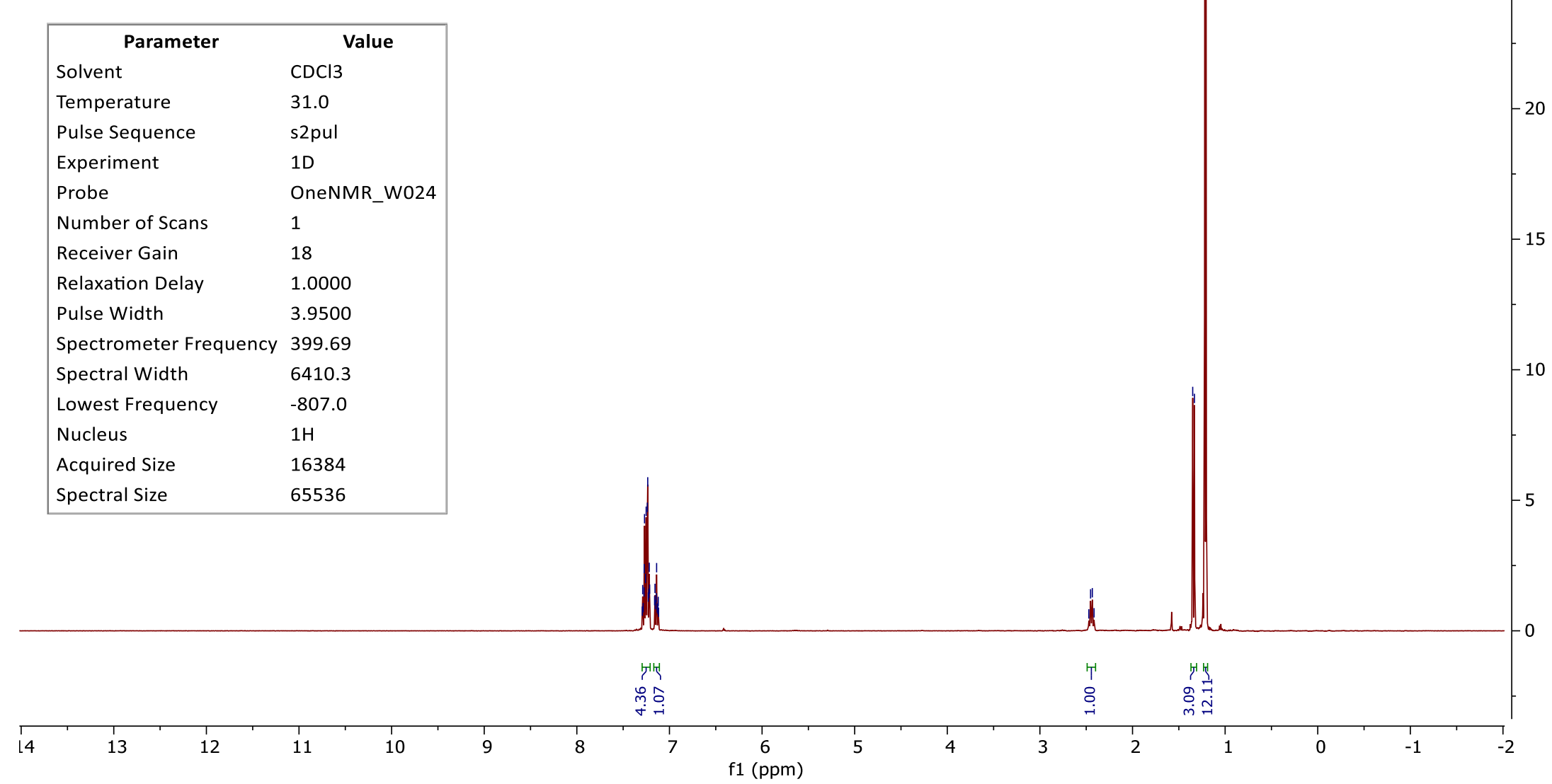



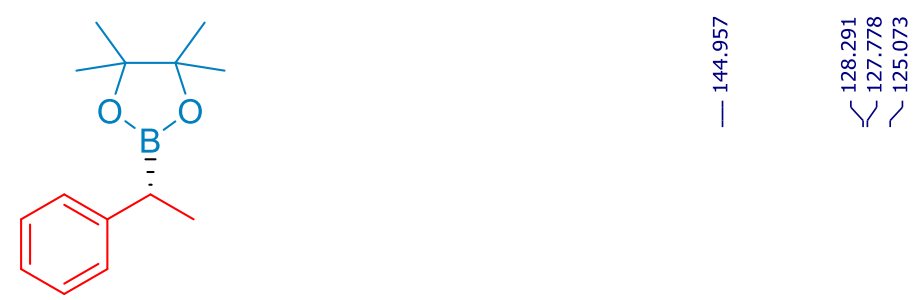

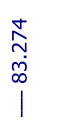

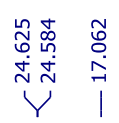

$-4.5$

$3 a$

\begin{tabular}{|ll|}
\hline \multicolumn{1}{|c|}{ Parameter } & \multicolumn{1}{c|}{ Value } \\
Solvent & CDCll3 \\
Temperature & 31.0 \\
Pulse Sequence & S2pul \\
Experiment & $1 \mathrm{D}$ \\
Probe & OneNMR_W024 \\
Number of Scans & 16 \\
Receiver Gain & 30 \\
Relaxation Delay & 1.0000 \\
Pulse Width & 4.9500 \\
Spectrometer Frequency & 100.51 \\
Spectral Width & 25000.0 \\
Lowest Frequency & -1444.7 \\
Nucleus & $13 \mathrm{C}$ \\
Acquired Size & 32768 \\
Spectral Size & 65536 \\
\hline
\end{tabular}

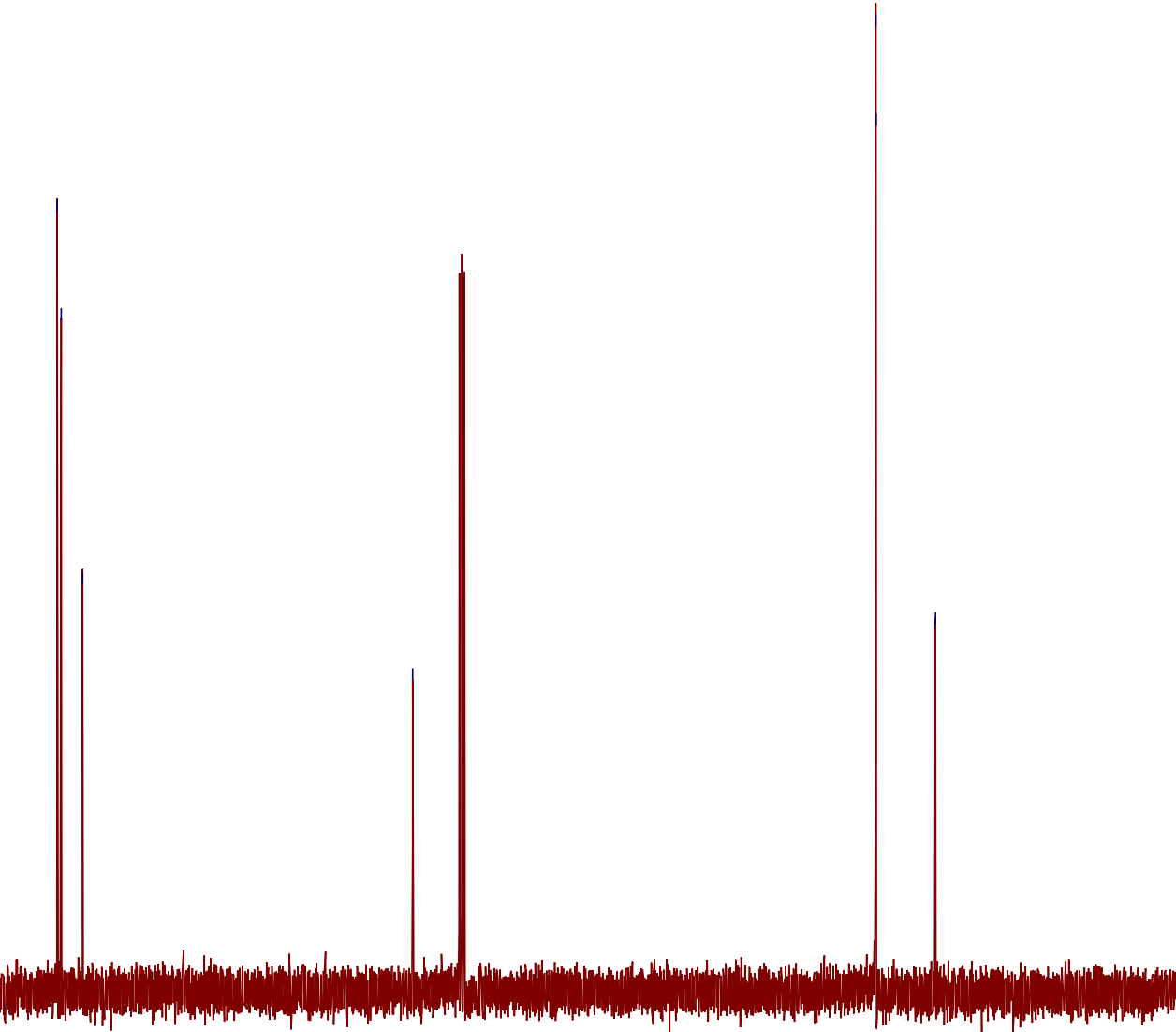

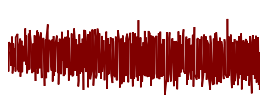

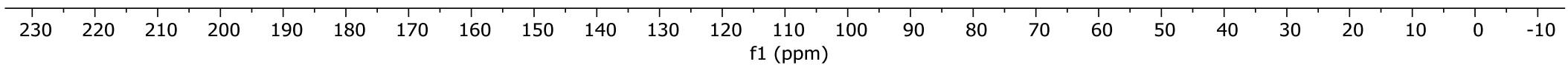




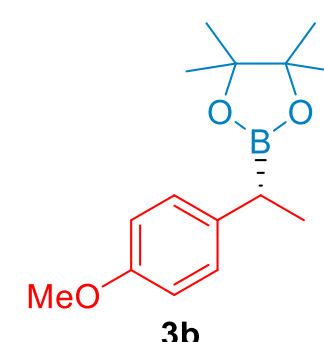

$3 b$

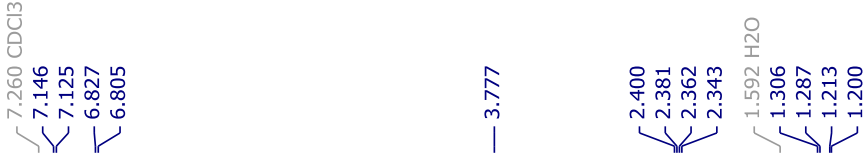

100

\begin{tabular}{|ll|}
\hline \multicolumn{1}{|c|}{ Parameter } & \multicolumn{1}{c|}{ Value } \\
Solvent & $\mathrm{CDCl} 3$ \\
Temperature & 31.0 \\
Pulse Sequence & s2pul \\
Experiment & $1 \mathrm{D}$ \\
Probe & OneNMR_W024 \\
Number of Scans & 1 \\
Receiver Gain & 30 \\
Relaxation Delay & 1.0000 \\
Pulse Width & 3.9500 \\
Spectrometer Frequency & 399.69 \\
Spectral Width & 6410.3 \\
Lowest Frequency & -806.3 \\
Nucleus & $1 \mathrm{H}$ \\
Acquired Size & 16384 \\
Spectral Size & 65536 \\
\hline
\end{tabular}

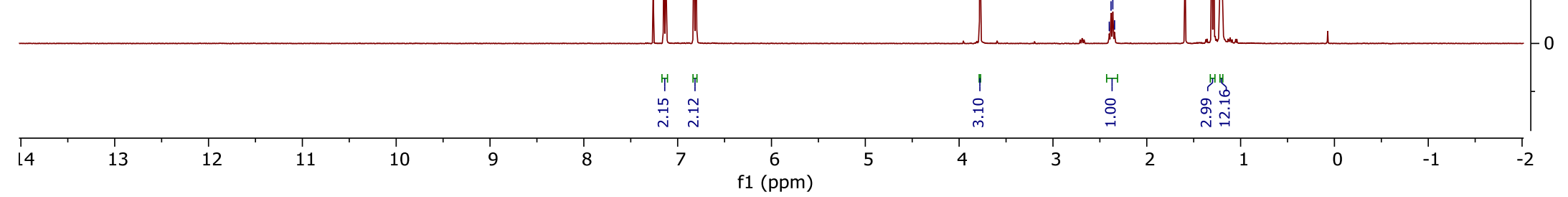



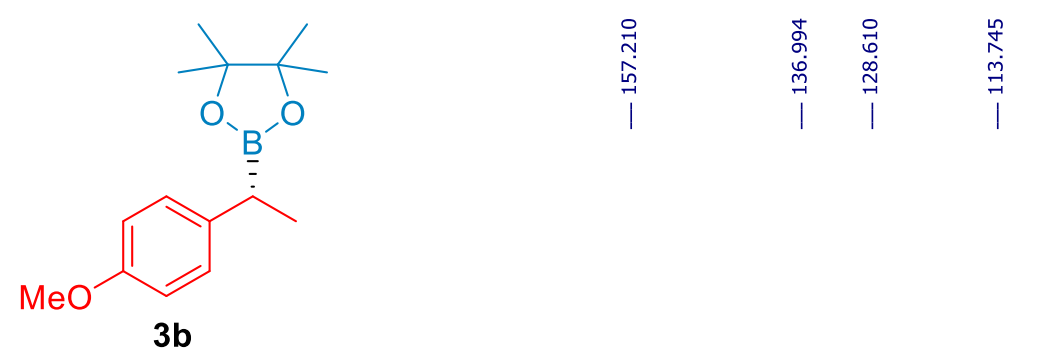

$\underset{\substack{N \\ \infty}}{\infty}$

先

$-34$

\begin{tabular}{|ll|}
\hline \multicolumn{1}{|c}{ Parameter } & \multicolumn{1}{c}{ Value } \\
Solvent & $\mathrm{CDCl} 3$ \\
Temperature & 31.0 \\
Pulse Sequence & s2pul \\
Experiment & $1 \mathrm{D}$ \\
Probe & OneNMR_W024 \\
Number of Scans & 256 \\
Receiver Gain & 30 \\
Relaxation Delay & 1.0000 \\
Pulse Width & 4.9500 \\
Spectrometer Frequency & 100.51 \\
Spectral Width & 25000.0 \\
Lowest Frequency & -1444.7 \\
Nucleus & $13 \mathrm{C}$ \\
Acquired Size & 32768 \\
Spectral Size & 65536 \\
\hline
\end{tabular}



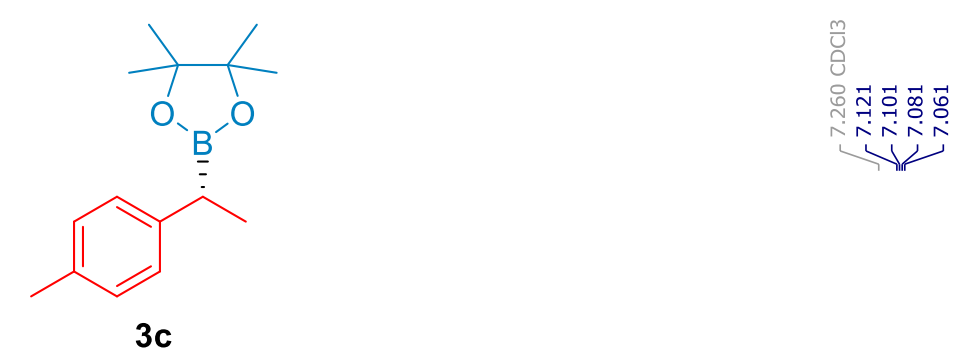

\begin{tabular}{|ll|}
\hline \multicolumn{1}{|c|}{ Parameter } & \multicolumn{1}{c|}{ Value } \\
Solvent & $\mathrm{CDCl} 3$ \\
Temperature & 31.0 \\
Pulse Sequence & s2pul \\
Experiment & $1 \mathrm{D}$ \\
Probe & OneNMR_W024 \\
Number of Scans & 8 \\
Receiver Gain & 30 \\
Relaxation Delay & 1.0000 \\
Pulse Width & 3.9500 \\
Spectrometer Frequency & 399.69 \\
Spectral Width & 6410.3 \\
Lowest Frequency & -806.4 \\
Nucleus & $1 \mathrm{H}$ \\
Acquired Size & 16384 \\
Spectral Size & 65536 \\
\hline
\end{tabular}

Spectral Size 65536

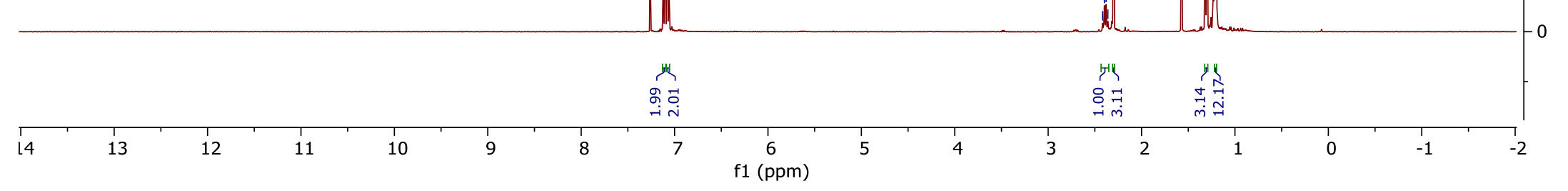



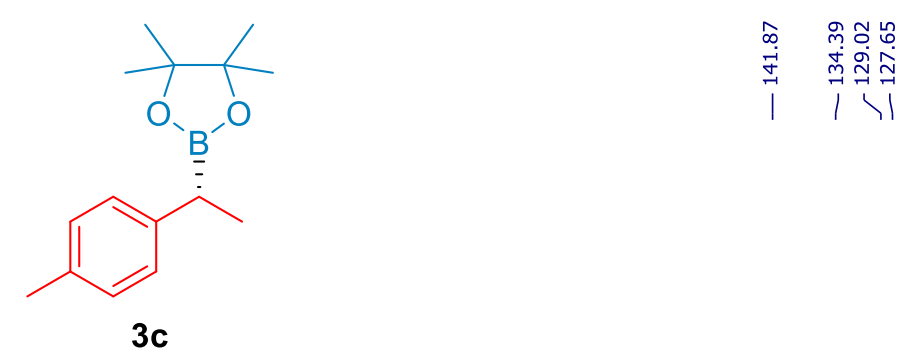

$\underset{\infty}{N}$

\begin{tabular}{|ll|}
\hline \multicolumn{1}{|c|}{ Parameter } & \multicolumn{1}{c|}{ Value } \\
Solvent & $\mathrm{CDCl} 3$ \\
Temperature & 31.0 \\
Pulse Sequence & s2pul \\
Experiment & $1 \mathrm{D}$ \\
Probe & OneNMR_W024 \\
Number of Scans & 256 \\
Receiver Gain & 30 \\
Relaxation Delay & 1.0000 \\
Pulse Width & 4.9500 \\
Spectrometer Frequency & 100.51 \\
Spectral Width & 25000.0 \\
Lowest Frequency & -1444.7 \\
Nucleus & $13 \mathrm{C}$ \\
Acquired Size & 32768 \\
Spectral Size & 65536 \\
\hline
\end{tabular}
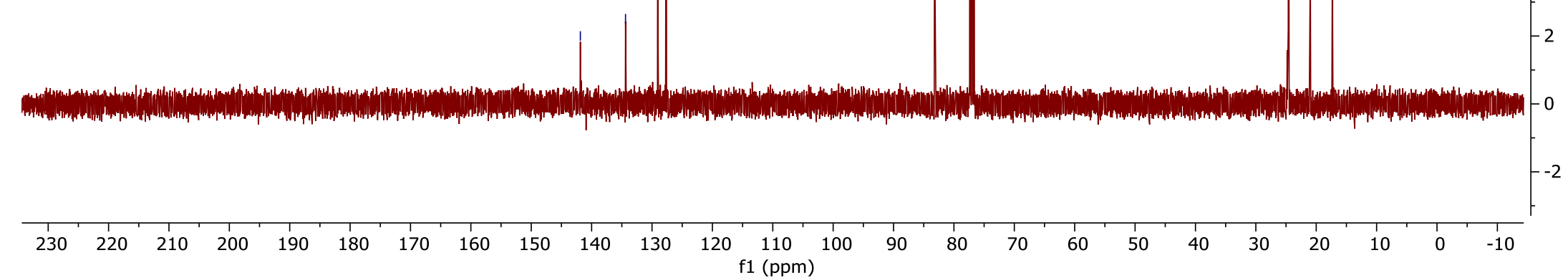

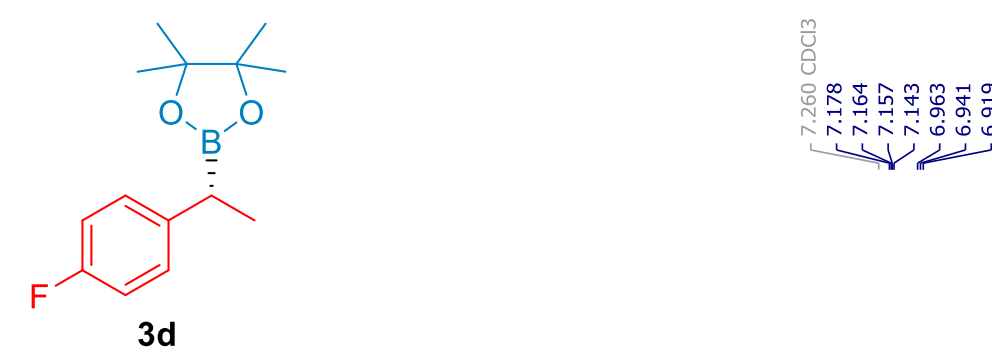

\begin{tabular}{|ll|}
\hline \multicolumn{1}{|c|}{ Parameter } & \multicolumn{1}{c|}{ Value } \\
Solvent & CDCl3 \\
Temperature & 31.0 \\
Pulse Sequence & s2pul \\
Experiment & $1 \mathrm{D}$ \\
Probe & OneNMR_W024 \\
Number of Scans & 8 \\
Receiver Gain & 30 \\
Relaxation Delay & 1.0000 \\
Pulse Width & 3.9500 \\
Spectrometer Frequency & 399.69 \\
Spectral Width & 6410.3 \\
Lowest Frequency & -806.2 \\
Nucleus & $1 \mathrm{H}$ \\
Acquired Size & 16384 \\
Spectral Size & 65536 \\
\hline
\end{tabular}

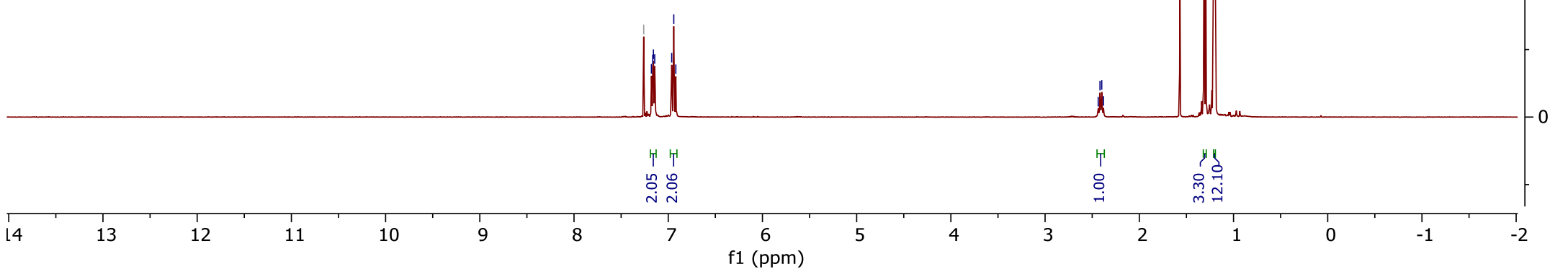



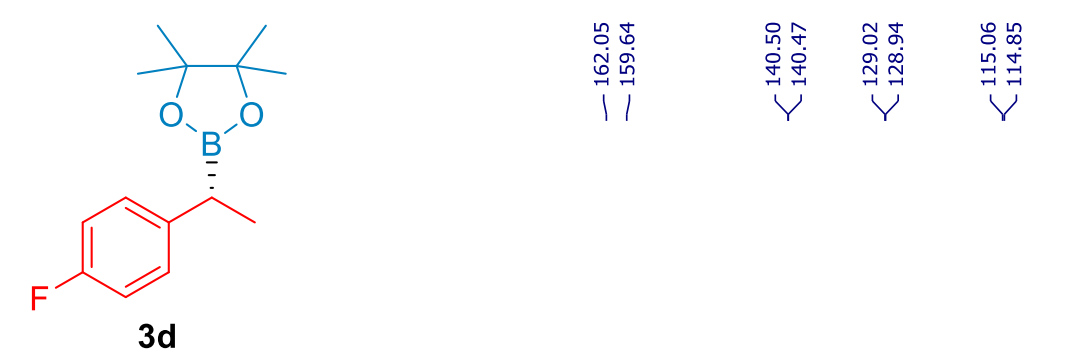

\begin{tabular}{|ll|}
\hline \multicolumn{1}{|c|}{ Parameter } & \multicolumn{1}{c|}{ Value } \\
Solvent & $\mathrm{CDCl} 3$ \\
Temperature & 31.0 \\
Pulse Sequence & s2pul \\
Experiment & $1 \mathrm{D}$ \\
Probe & OneNMR_W024 \\
Number of Scans & 256 \\
Receiver Gain & 30 \\
Relaxation Delay & 1.0000 \\
Pulse Width & 4.9500 \\
Spectrometer Frequency & 100.51 \\
Spectral Width & 25000.0 \\
Lowest Frequency & -1444.7 \\
Nucleus & $13 \mathrm{C}$ \\
Acquired Size & 32768 \\
Spectral Size & 65536 \\
\hline
\end{tabular}

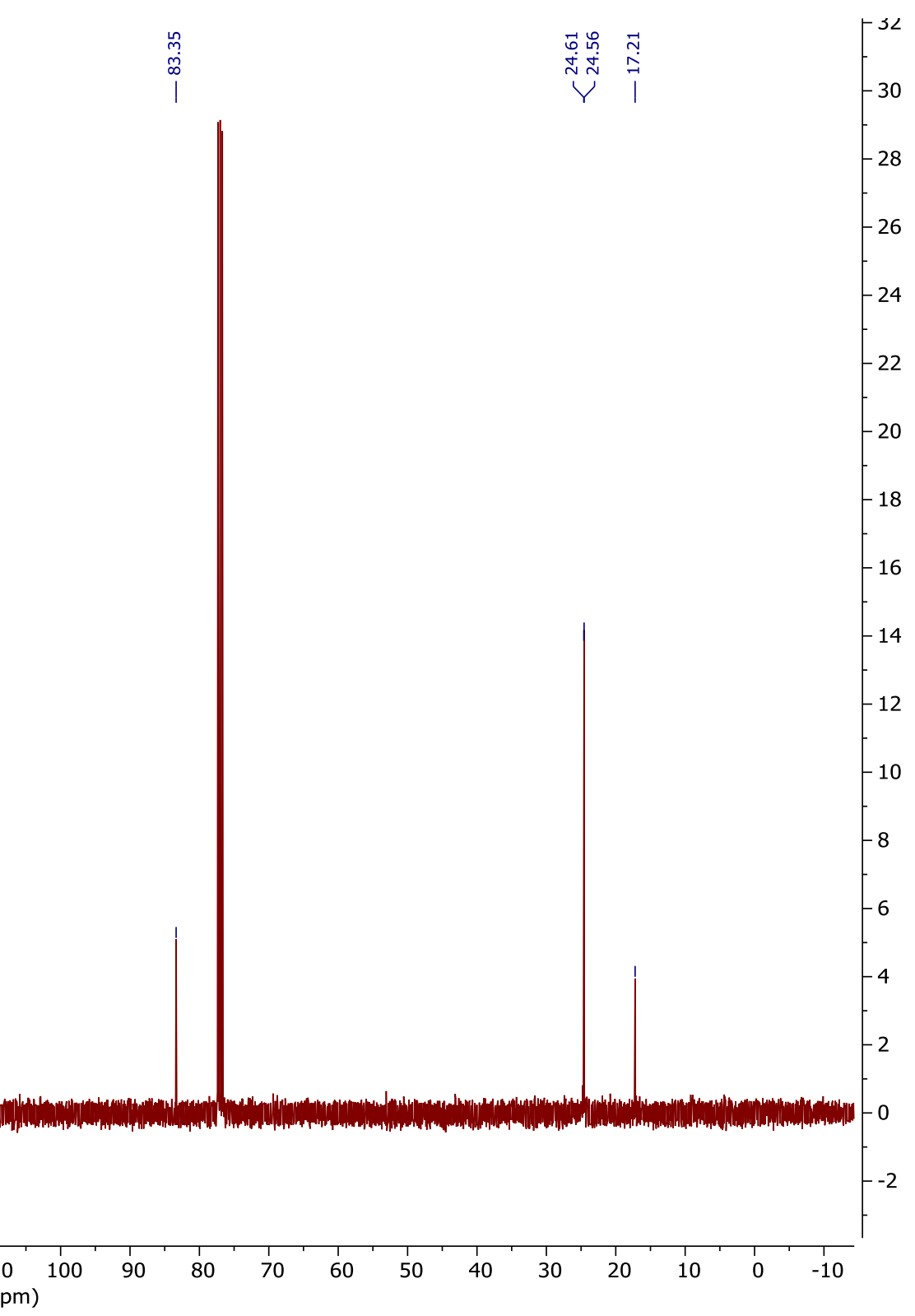




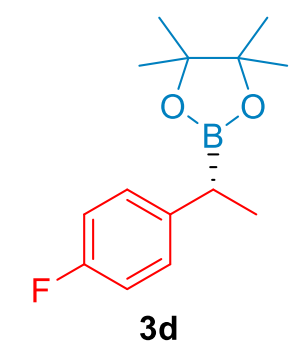

\begin{tabular}{ll|}
\multicolumn{1}{c}{ Parameter } & \multicolumn{1}{c|}{ Value } \\
Solvent & $\mathrm{CDCl} 3$ \\
Temperature & 31.0 \\
Pulse Sequence & s2pul \\
Experiment & $1 \mathrm{D}$ \\
Probe & OneNMR_W024 \\
Number of Scans & 16 \\
Receiver Gain & 30 \\
Relaxation Delay & 1.0000 \\
Pulse Width & 3.3333 \\
Spectrometer Frequency & 376.05 \\
Spectral Width & 89285.7 \\
Lowest Frequency & -76610.2 \\
Nucleus & $19 \mathrm{~F}$ \\
Acquired Size & 65536 \\
Spectral Size & 131072 \\
\hline
\end{tabular}

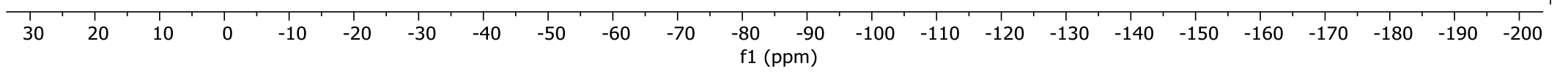



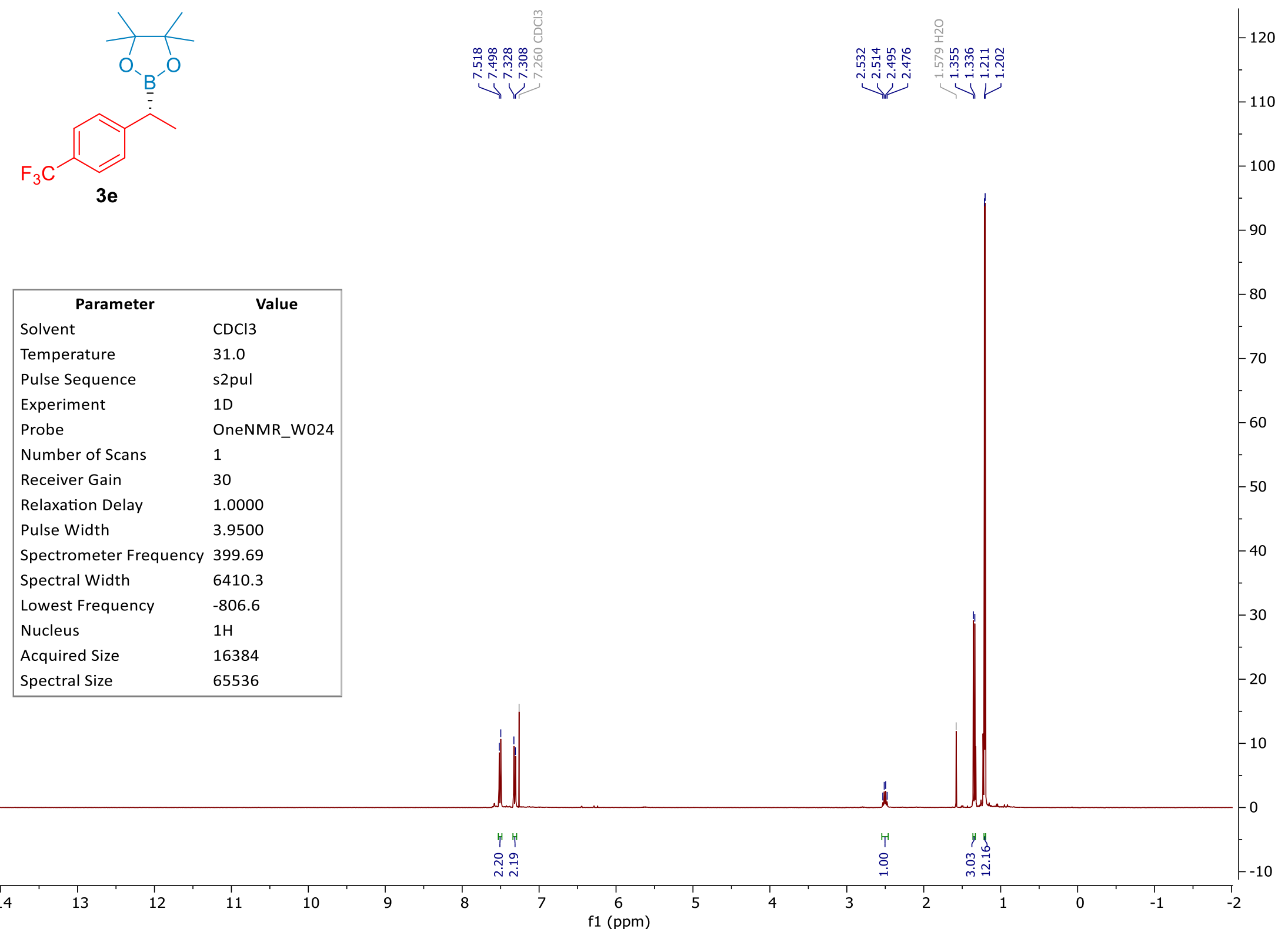

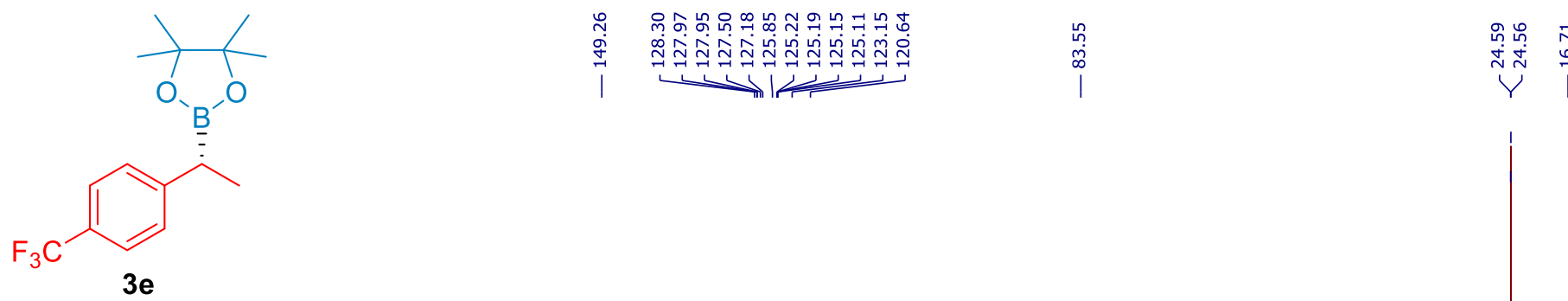

ํํㄴㅇㅠ 눈

$-23$

\begin{tabular}{|ll|}
\hline \multicolumn{1}{|c|}{ Parameter } & \multicolumn{1}{c|}{ Value } \\
Solvent & $\mathrm{CDCl} 3$ \\
Temperature & 31.0 \\
Pulse Sequence & s2pul \\
Experiment & $1 \mathrm{D}$ \\
Probe & OneNMR_W024 \\
Number of Scans & 256 \\
Receiver Gain & 24 \\
Relaxation Delay & 1.0000 \\
Pulse Width & 4.9500 \\
Spectrometer Frequency & 100.51 \\
Spectral Width & 25000.0 \\
Lowest Frequency & -1444.7 \\
Nucleus & $13 \mathrm{C}$ \\
Acquired Size & 32768 \\
Spectral Size & 65536 \\
\hline
\end{tabular}
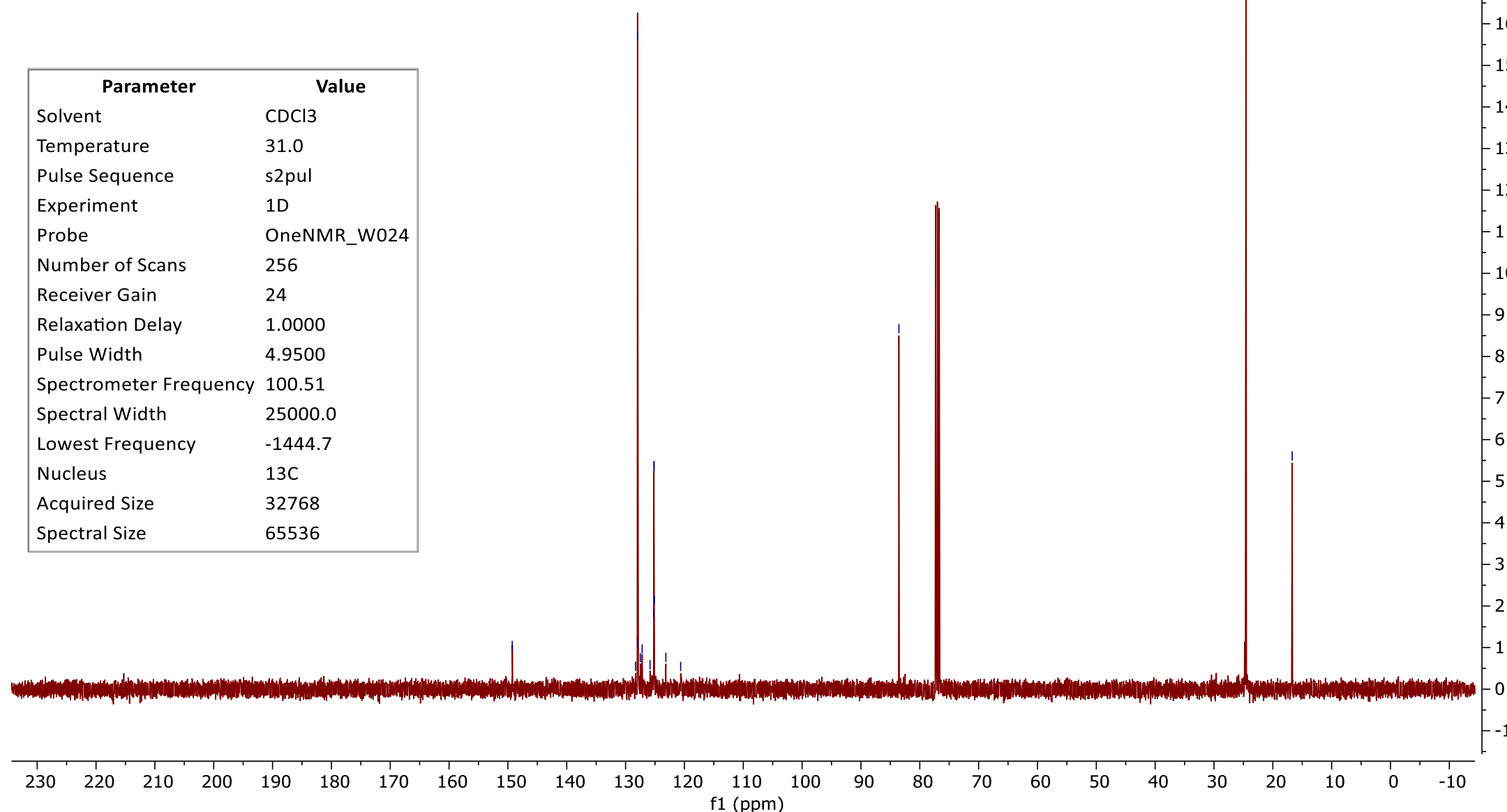


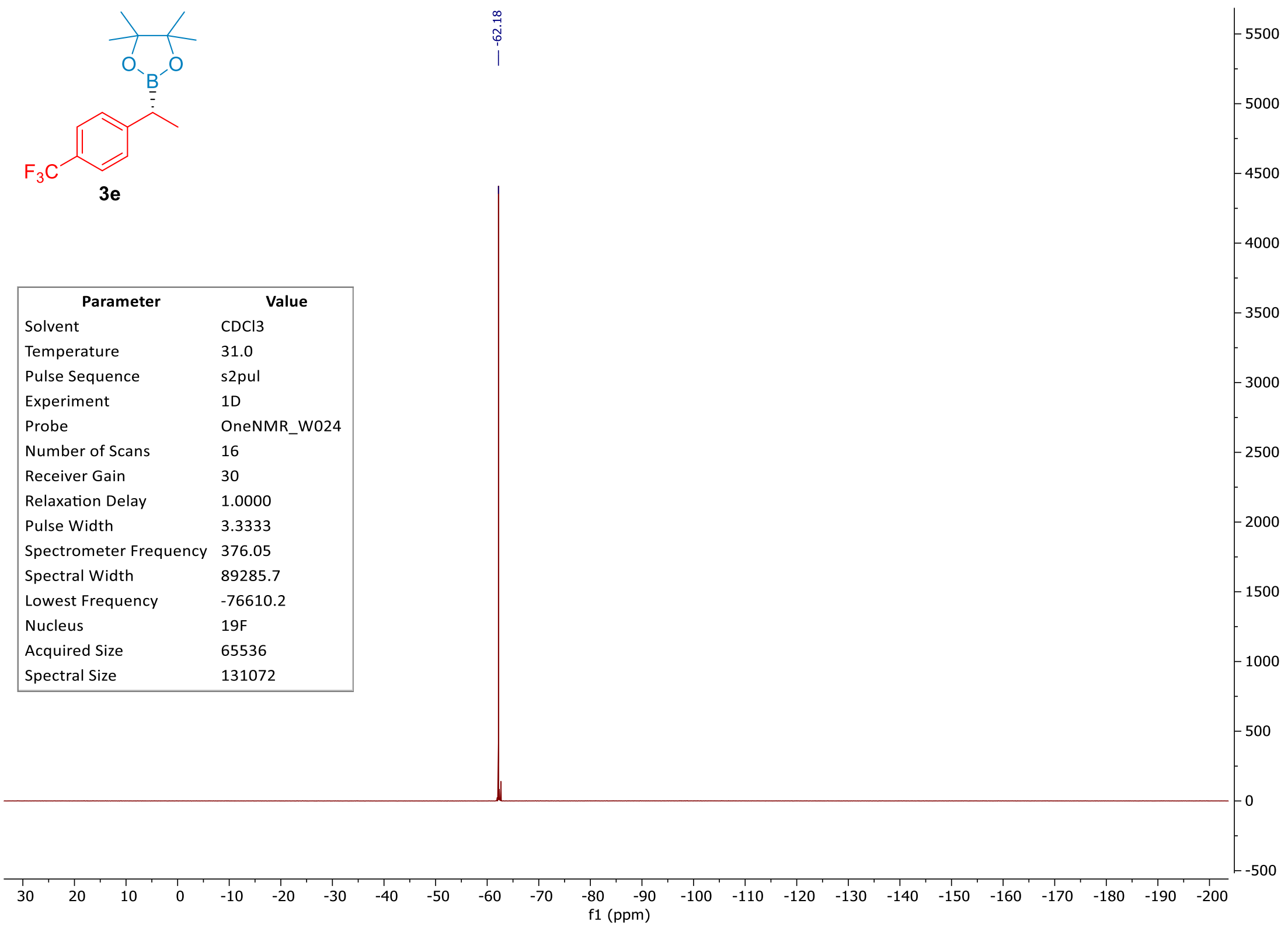




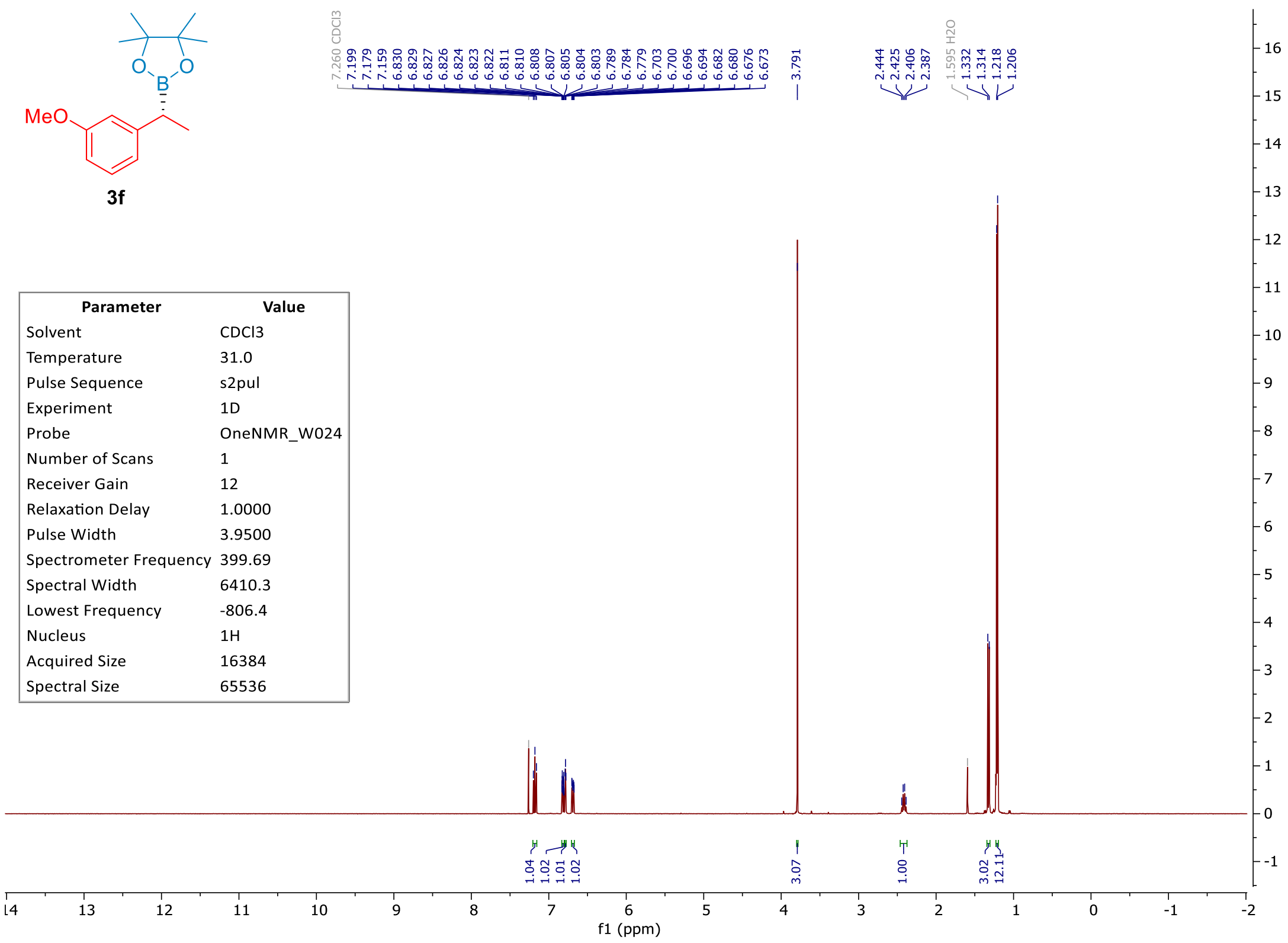




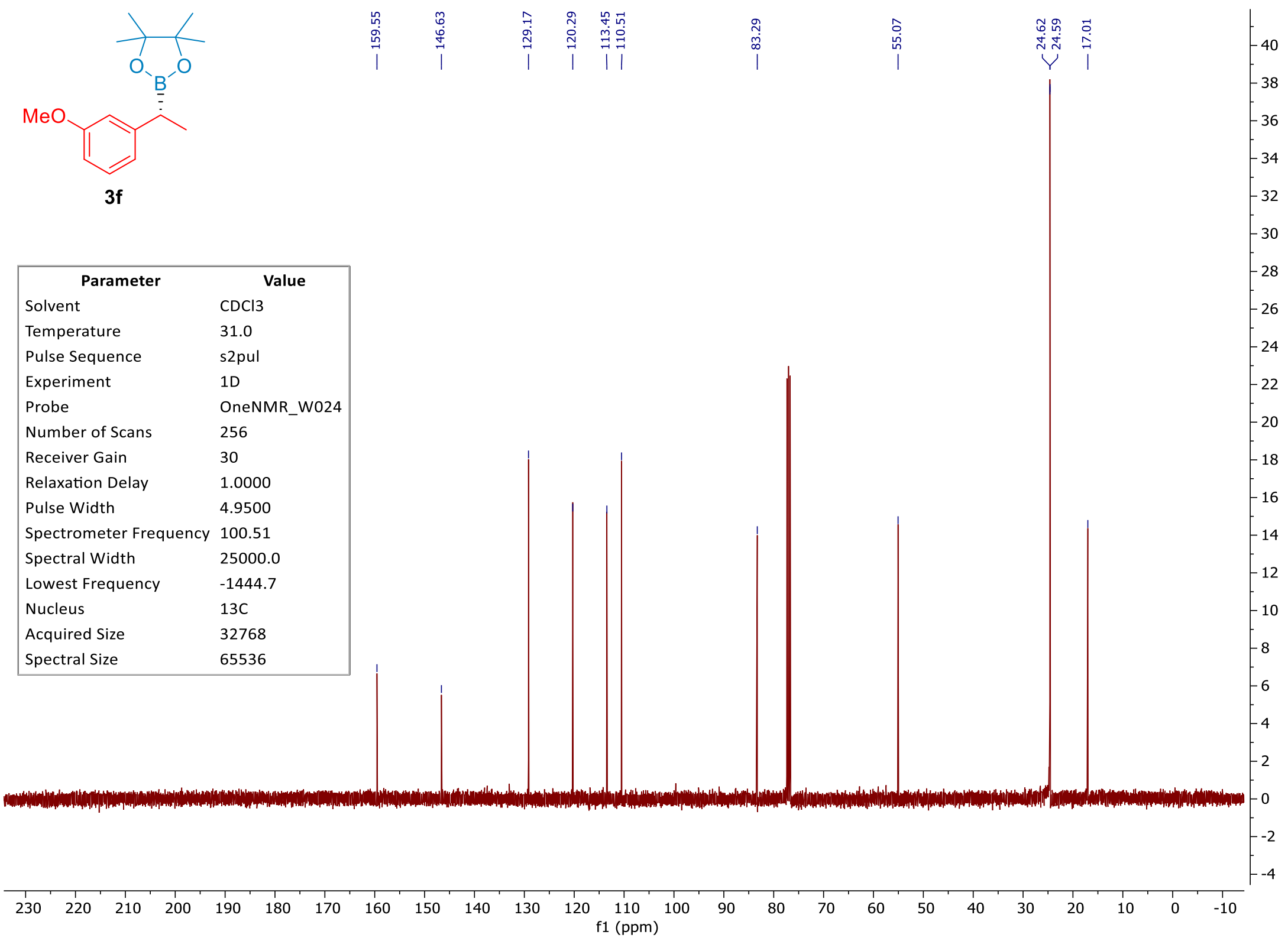




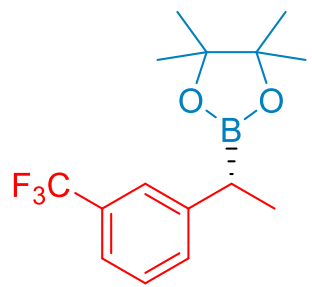

$3 g$

\begin{tabular}{|ll|}
\hline \multicolumn{1}{|c|}{ Parameter } & \multicolumn{1}{c|}{ Value } \\
Solvent & $\mathrm{CDCl3}$ \\
Temperature & 31.0 \\
Pulse Sequence & s2pul \\
Experiment & $1 \mathrm{D}$ \\
Probe & OneNMR_W024 \\
Number of Scans & 8 \\
Receiver Gain & 30 \\
Relaxation Delay & 1.0000 \\
Pulse Width & 3.9500 \\
Spectrometer Frequency & 399.69 \\
Spectral Width & 6410.3 \\
Lowest Frequency & -806.4 \\
Nucleus & $1 \mathrm{H}$ \\
Acquired Size & 16384 \\
Spectral Size & 65536 \\
\hline
\end{tabular}




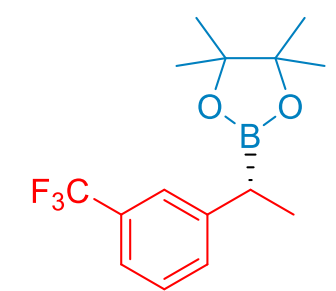

3g

\begin{tabular}{|ll|}
\hline \multicolumn{1}{|c|}{ Parameter } & \multicolumn{1}{c|}{ Value } \\
Solvent & $\mathrm{CDCl} 3$ \\
Temperature & 31.0 \\
Pulse Sequence & s2pul \\
Experiment & $1 \mathrm{D}$ \\
Probe & OneNMR_W024 \\
Number of Scans & 256 \\
Receiver Gain & 30 \\
Relaxation Delay & 1.0000 \\
Pulse Width & 4.9500 \\
Spectrometer Frequency & 100.51 \\
Spectral Width & 25000.0 \\
Lowest Frequency & -1444.7 \\
Nucleus & $13 \mathrm{C}$ \\
Acquired Size & 32768 \\
Spectral Size & 65536 \\
\hline
\end{tabular}

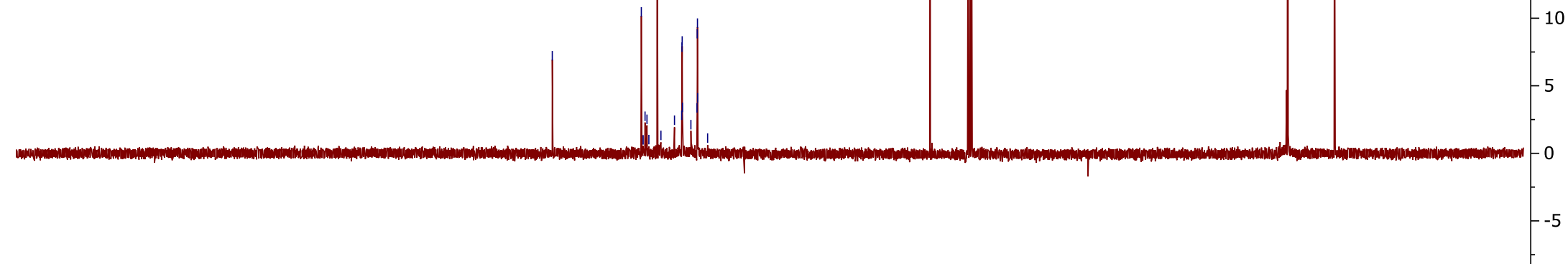

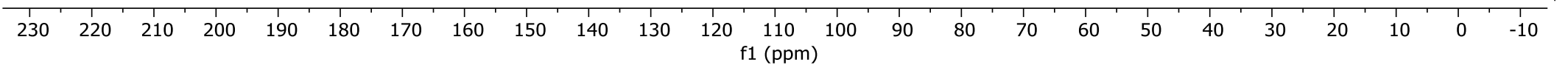




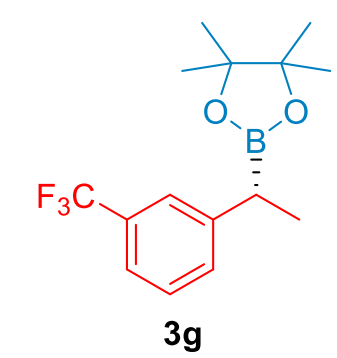

$-5000$

$-4500$

\begin{tabular}{|ll|}
\hline \multicolumn{1}{|c|}{ Parameter } & \multicolumn{1}{c|}{ Value } \\
Solvent & $\mathrm{CDCl} 3$ \\
Temperature & 31.0 \\
Pulse Sequence & s2pul \\
Experiment & $1 \mathrm{D}$ \\
Probe & OneNMR_W024 \\
Number of Scans & 16 \\
Receiver Gain & 30 \\
Relaxation Delay & 1.0000 \\
Pulse Width & 3.3333 \\
Spectrometer Frequency & 376.05 \\
Spectral Width & 89285.7 \\
Lowest Frequency & -76610.2 \\
Nucleus & $19 \mathrm{~F}$ \\
Acquired Size & 65536 \\
Spectral Size & 131072 \\
\hline
\end{tabular}

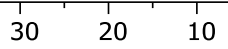

$\begin{array}{ll}-10 & -20\end{array}$

$-40 \quad-50$

$\begin{array}{ll}-60 & -70\end{array}$

$\begin{array}{ll}-80 & -90\end{array}$

f1 (ppm) 

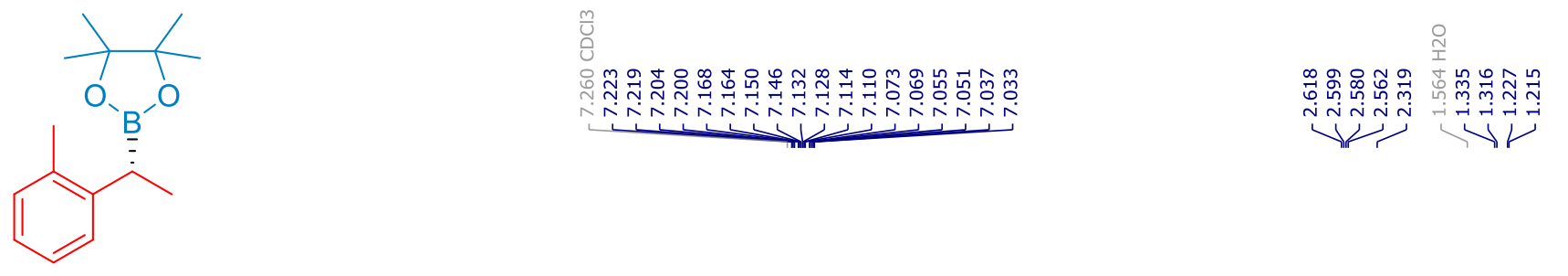

$3 \mathrm{~h}$

\begin{tabular}{|ll|}
\hline \multicolumn{1}{|c}{ Parameter } & \multicolumn{1}{c|}{ Value } \\
Solvent & $\mathrm{CDCl} 3$ \\
Temperature & 31.0 \\
Pulse Sequence & s2pul \\
Experiment & $1 \mathrm{D}$ \\
Probe & OneNMR_W024 \\
Number of Scans & 8 \\
Receiver Gain & 30 \\
Relaxation Delay & 1.0000 \\
Pulse Width & 3.9500 \\
Spectrometer Frequency & 399.69 \\
Spectral Width & 6410.3 \\
Lowest Frequency & -806.6 \\
Nucleus & $1 \mathrm{H}$ \\
Acquired Size & 16384 \\
Spectral Size & 65536 \\
\hline
\end{tabular}

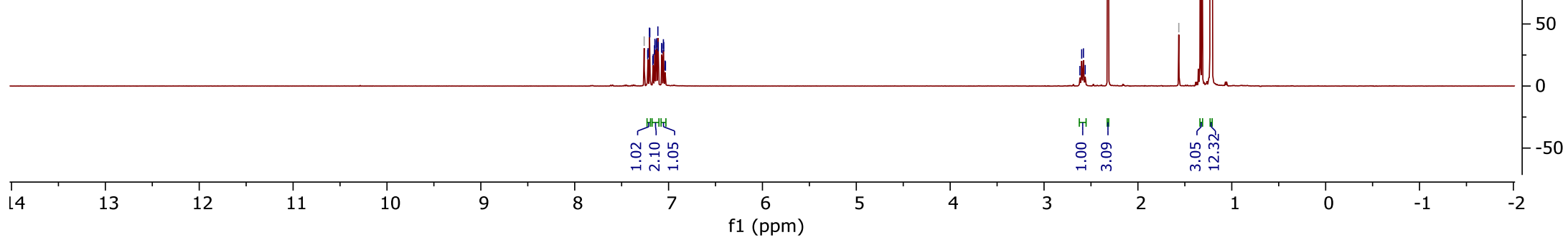



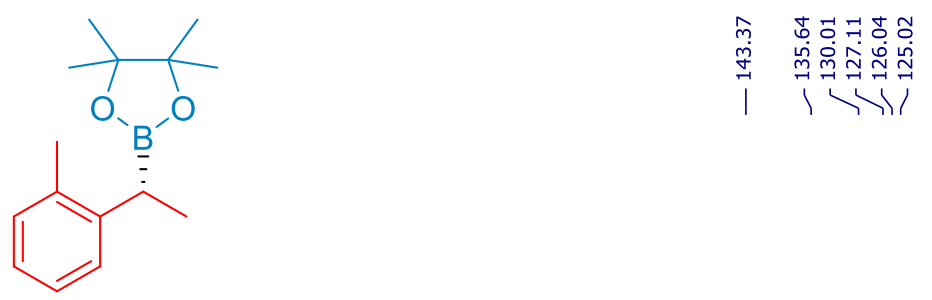

$3 \mathrm{~h}$

\begin{tabular}{|ll|}
\hline \multicolumn{1}{|c|}{ Parameter } & \multicolumn{1}{c|}{ Value } \\
Solvent & $\mathrm{CDCl} 3$ \\
Temperature & 31.0 \\
Pulse Sequence & s2pul \\
Experiment & $1 \mathrm{D}$ \\
Probe & OneNMR_W024 \\
Number of Scans & 256 \\
Receiver Gain & 30 \\
Relaxation Delay & 1.0000 \\
Pulse Width & 4.9500 \\
Spectrometer Frequency & 100.51 \\
Spectral Width & 25000.0 \\
Lowest Frequency & -1444.7 \\
Nucleus & $13 \mathrm{C}$ \\
Acquired Size & 32768 \\
Spectral Size & 65536 \\
\hline
\end{tabular}

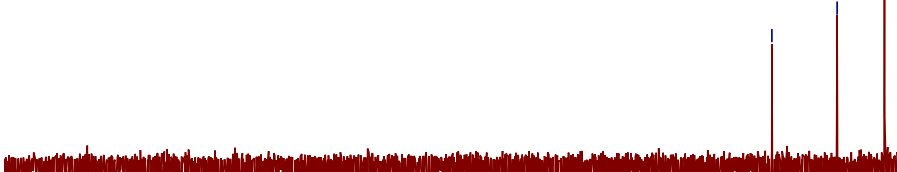

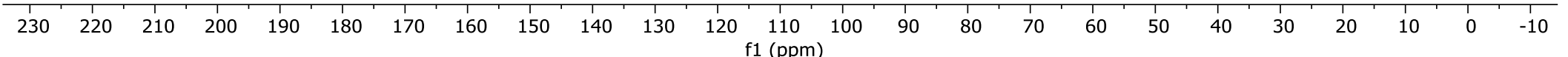



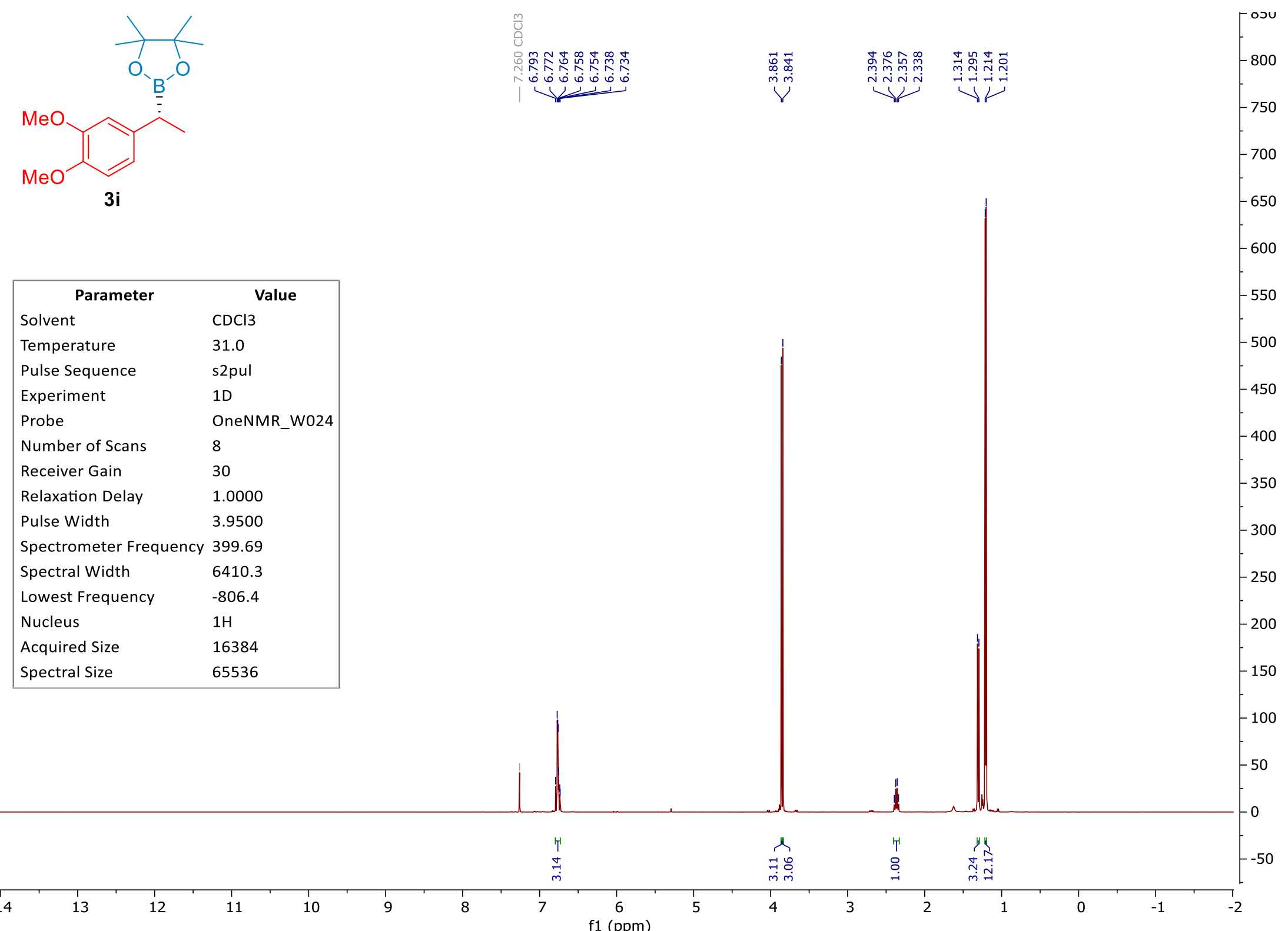

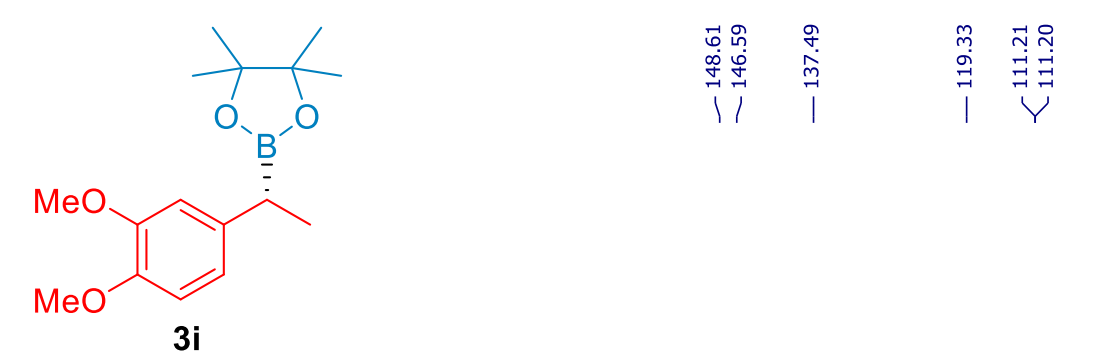

$\stackrel{\substack{n \\ \infty}}{\infty}$

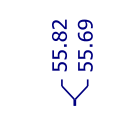

\begin{tabular}{|ll|}
\hline \multicolumn{1}{|c|}{ Parameter } & \multicolumn{1}{c|}{ Value } \\
Solvent & $\mathrm{CDCl} 3$ \\
Temperature & 31.0 \\
Pulse Sequence & s2pul \\
Experiment & $1 \mathrm{D}$ \\
Probe & OneNMR_W024 \\
Number of Scans & 256 \\
Receiver Gain & 30 \\
Relaxation Delay & 1.0000 \\
Pulse Width & 4.9500 \\
Spectrometer Frequency & 100.51 \\
Spectral Width & 25000.0 \\
Lowest Frequency & -1444.7 \\
Nucleus & $13 \mathrm{C}$ \\
Acquired Size & 32768 \\
Spectral Size & 65536 \\
\hline
\end{tabular}

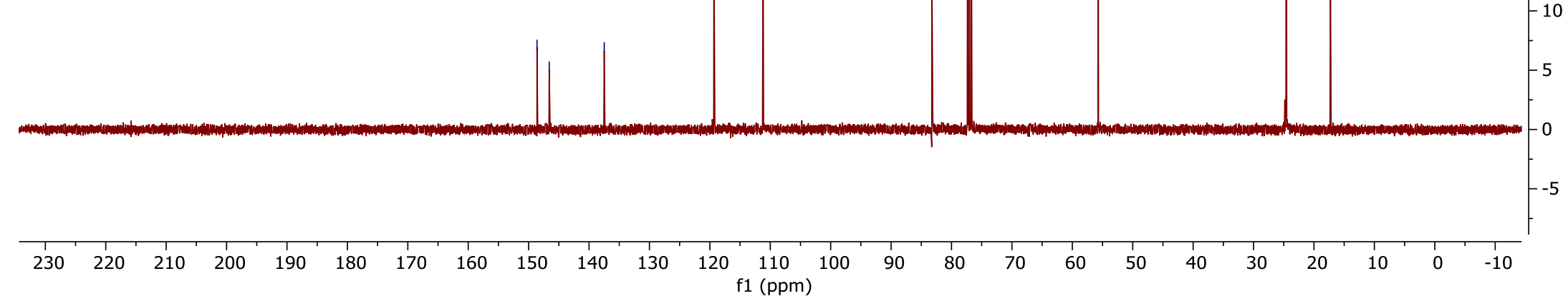



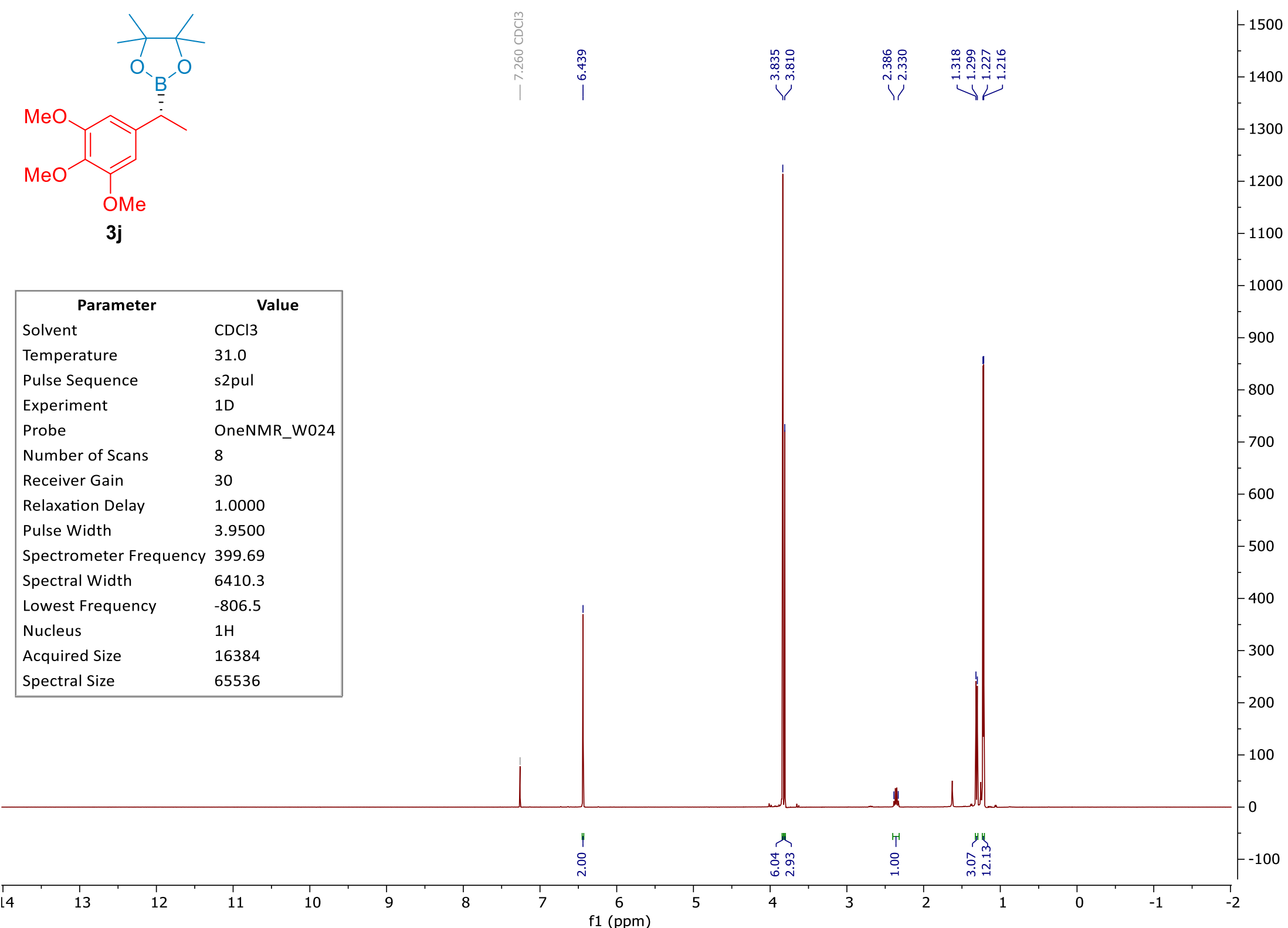


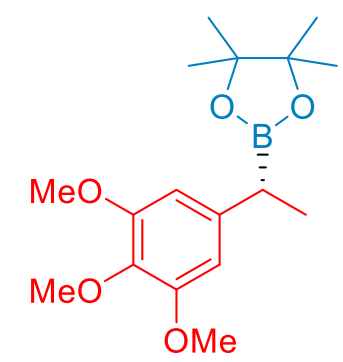

\begin{tabular}{|ll|}
\hline \multicolumn{1}{|c|}{ Parameter } & \multicolumn{1}{c|}{ Value } \\
Solvent & $\mathrm{CDCl} 3$ \\
Temperature & 31.0 \\
Pulse Sequence & s2pul \\
Experiment & $1 \mathrm{D}$ \\
Probe & OneNMR_W024 \\
Number of Scans & 256 \\
Receiver Gain & 30 \\
Relaxation Delay & 1.0000 \\
Pulse Width & 4.9500 \\
Spectrometer Frequency & 100.51 \\
Spectral Width & 25000.0 \\
Lowest Frequency & -1444.7 \\
Nucleus & $13 \mathrm{C}$ \\
Acquired Size & 32768 \\
Spectral Size & 65536 \\
\hline
\end{tabular}

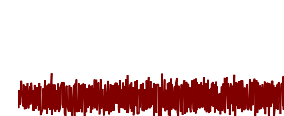

.




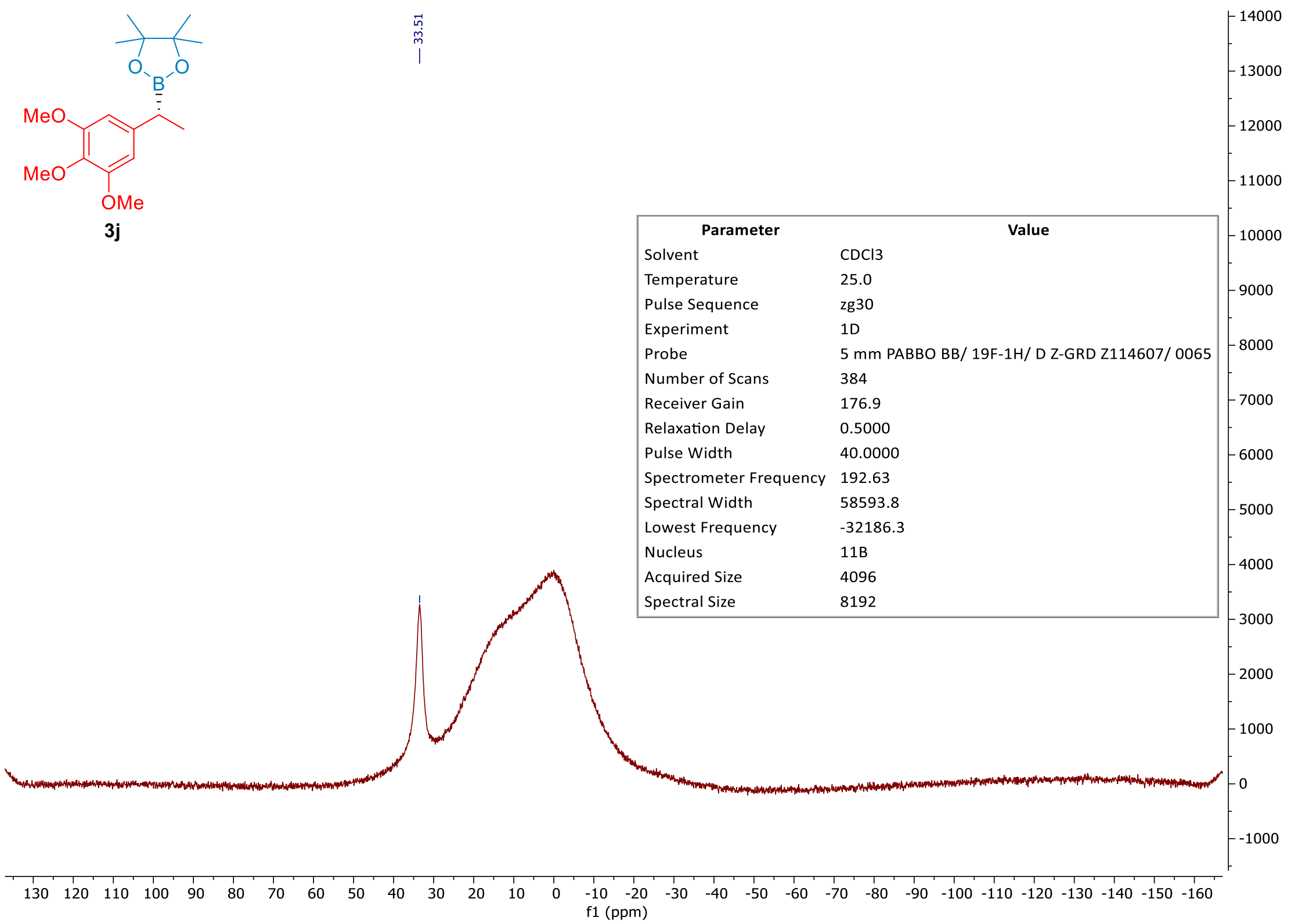



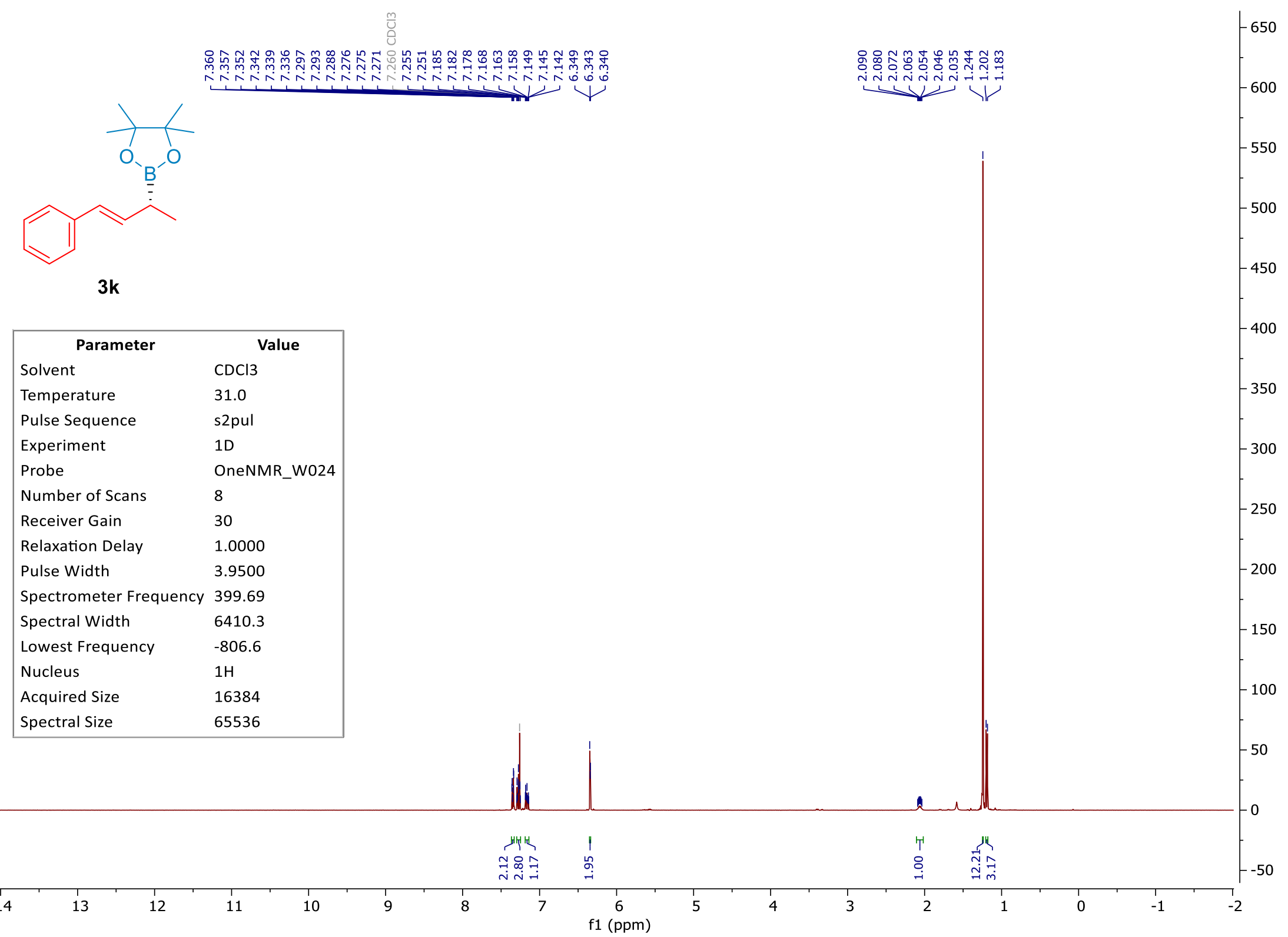

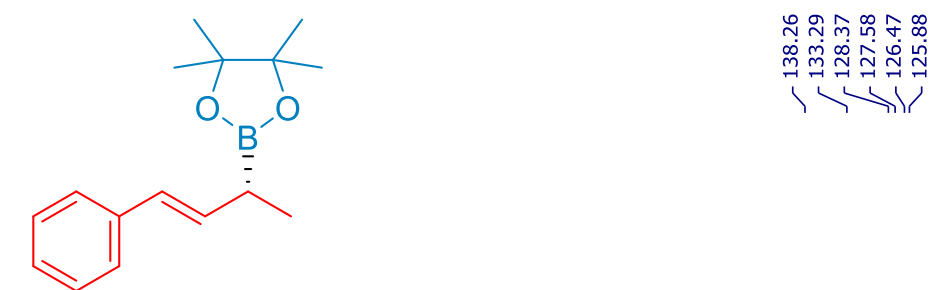

$3 \mathbf{k}$

\begin{tabular}{|ll|}
\hline \multicolumn{1}{|c|}{ Parameter } & \multicolumn{1}{c|}{ Value } \\
Solvent & $\mathrm{CDCl} 3$ \\
Temperature & 31.0 \\
Pulse Sequence & s2pul \\
Experiment & $1 \mathrm{D}$ \\
Probe & OneNMR_W024 \\
Number of Scans & 256 \\
Receiver Gain & 30 \\
Relaxation Delay & 1.0000 \\
Pulse Width & 4.9500 \\
Spectrometer Frequency & 100.51 \\
Spectral Width & 25000.0 \\
Lowest Frequency & -1444.7 \\
Nucleus & $13 \mathrm{C}$ \\
Acquired Size & 32768 \\
Spectral Size & 65536 \\
\hline
\end{tabular}

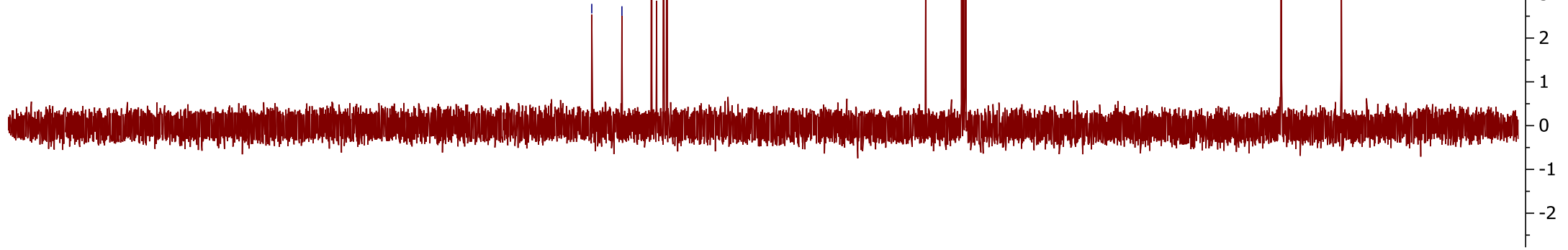

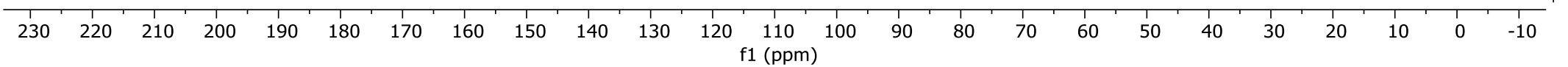




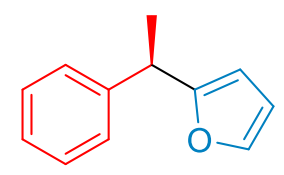

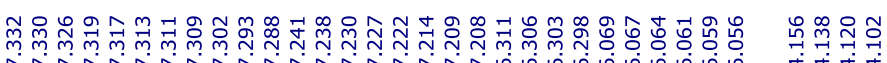

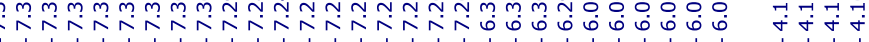

4aa

\begin{tabular}{|ll|}
\hline \multicolumn{1}{|c|}{ Parameter } & \multicolumn{1}{c|}{ Value } \\
Solvent & $\mathrm{CDCl} 3$ \\
Temperature & 31.0 \\
Pulse Sequence & s2pul \\
Experiment & $1 \mathrm{D}$ \\
Probe & OneNMR_W024 \\
Number of Scans & 8 \\
Receiver Gain & 30 \\
Relaxation Delay & 1.0000 \\
Pulse Width & 3.9500 \\
Spectrometer Frequency & 399.69 \\
Spectral Width & 6410.3 \\
Lowest Frequency & -806.6 \\
Nucleus & $1 \mathrm{H}$ \\
Acquired Size & 16384 \\
Spectral Size & 65536 \\
\hline
\end{tabular}

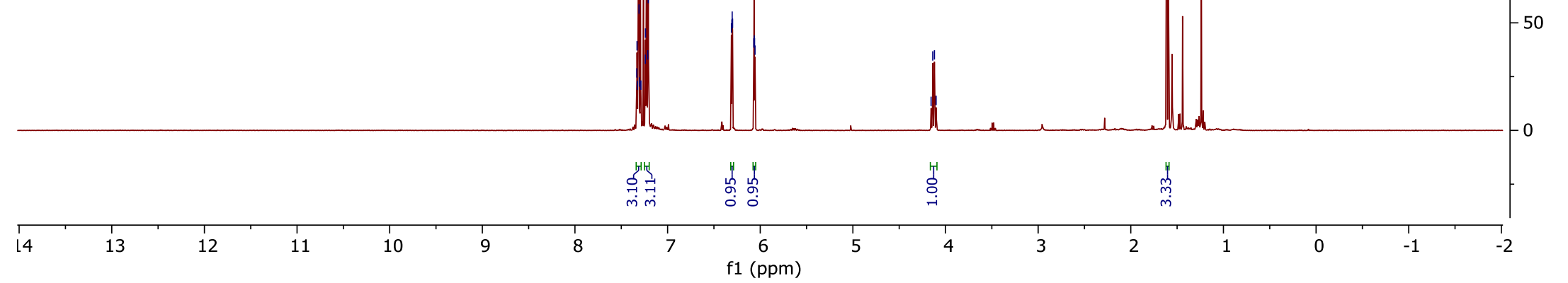




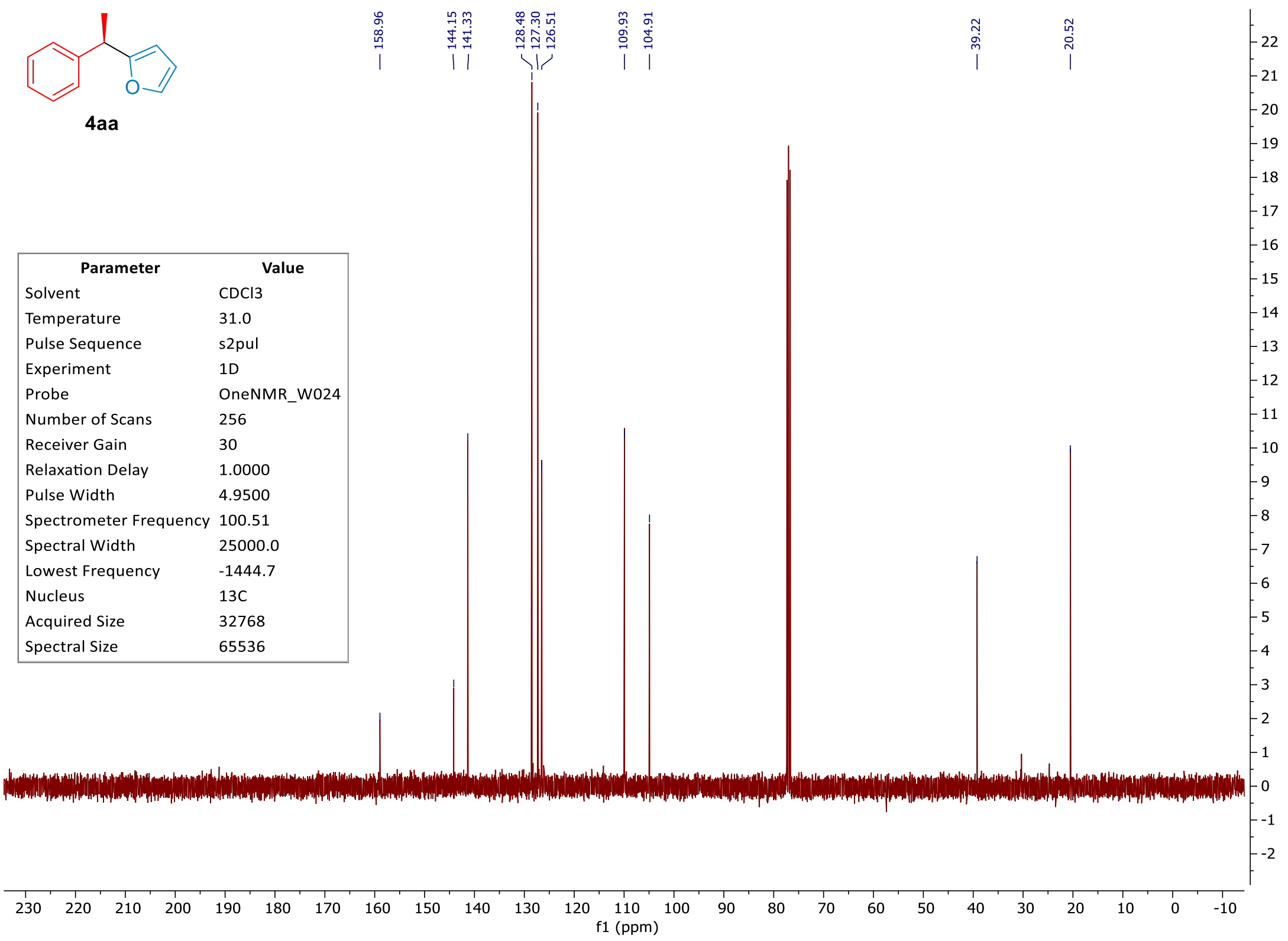




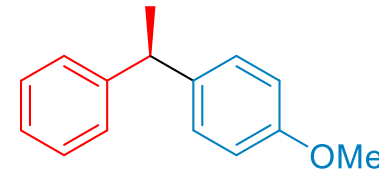

$4 a b$

OMe

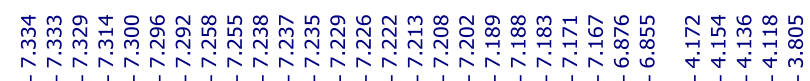

Temperature

$\mathrm{CDCl} 3$

Pulse Sequence

31.0

Experiment

s2pul

Probe

1D

Number of Scans

Receiver Gain

OneNMR_W024

Relaxation Delay

8

Pulse Width

30

1.0000

Spectrometer Frequency 399.69

Spectral Width

6410.3

Lowest Frequency

$-807.0$

Nucleus

Acquired Size

$1 \mathrm{H}$

Spectral Size

16384

65536

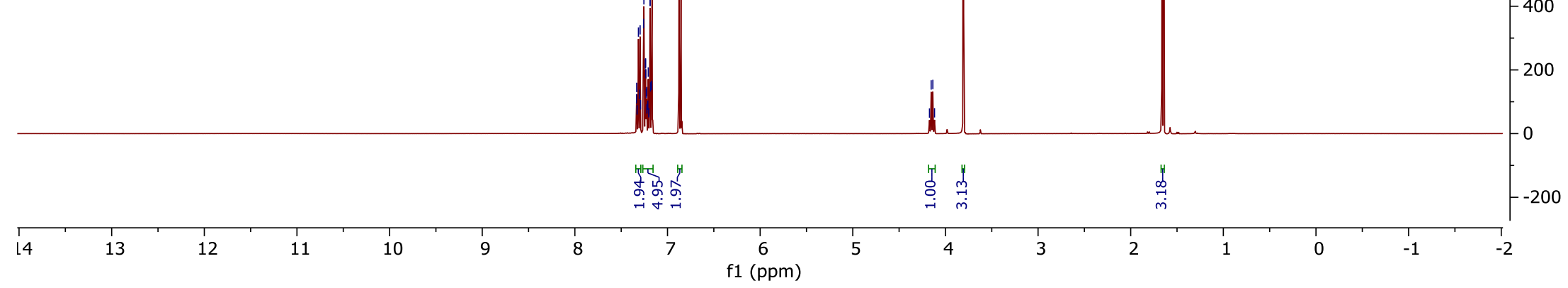




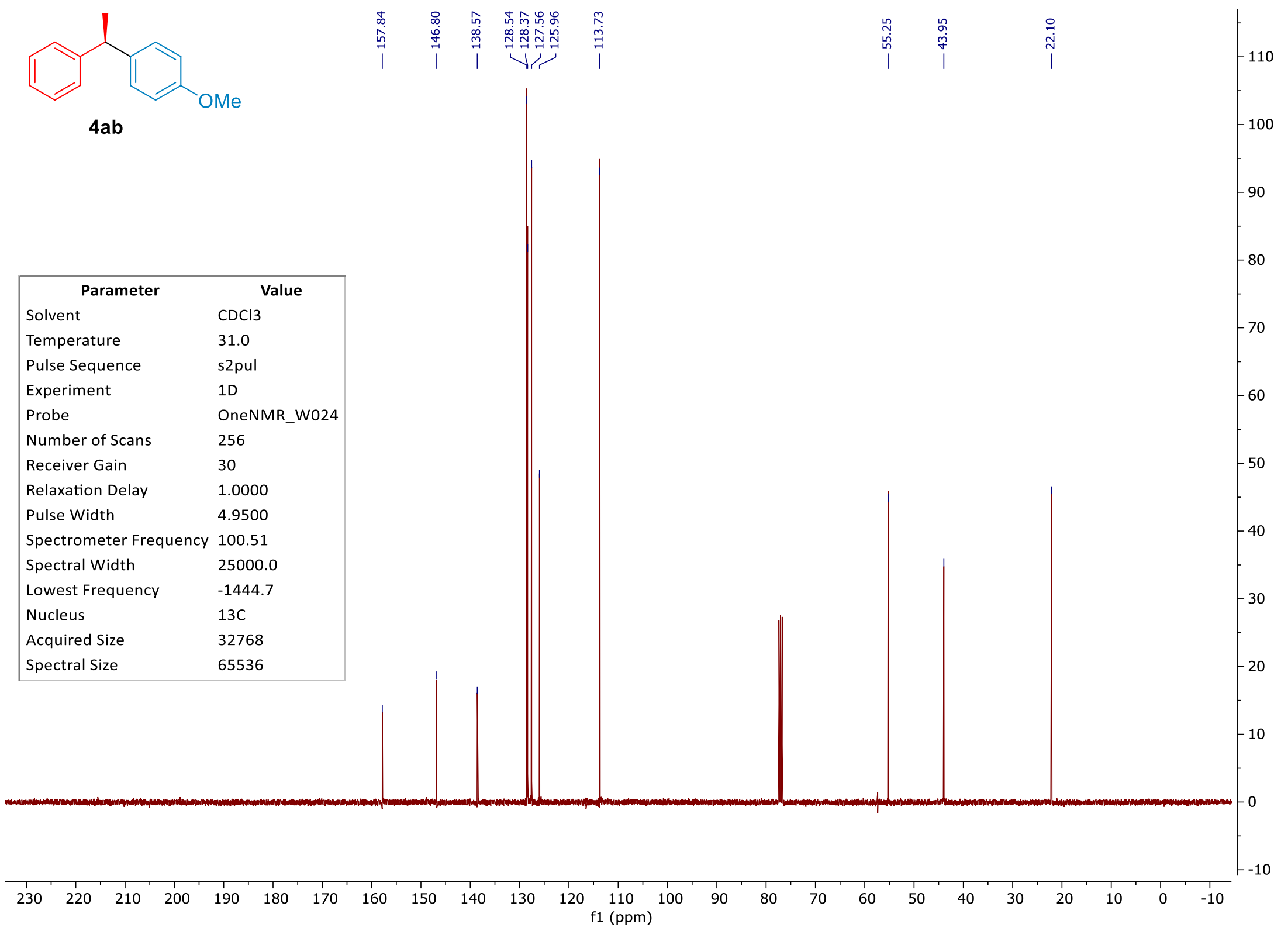



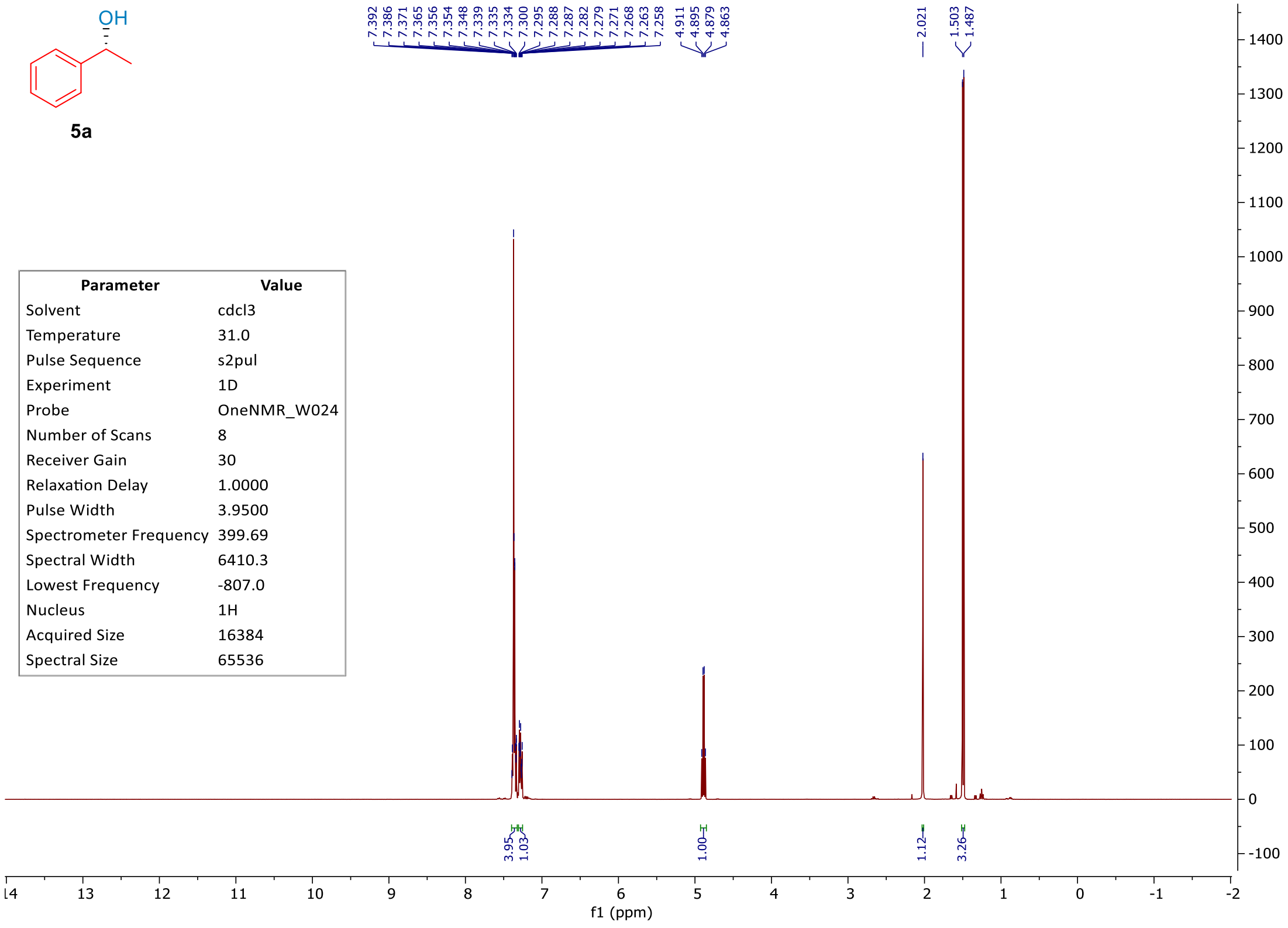


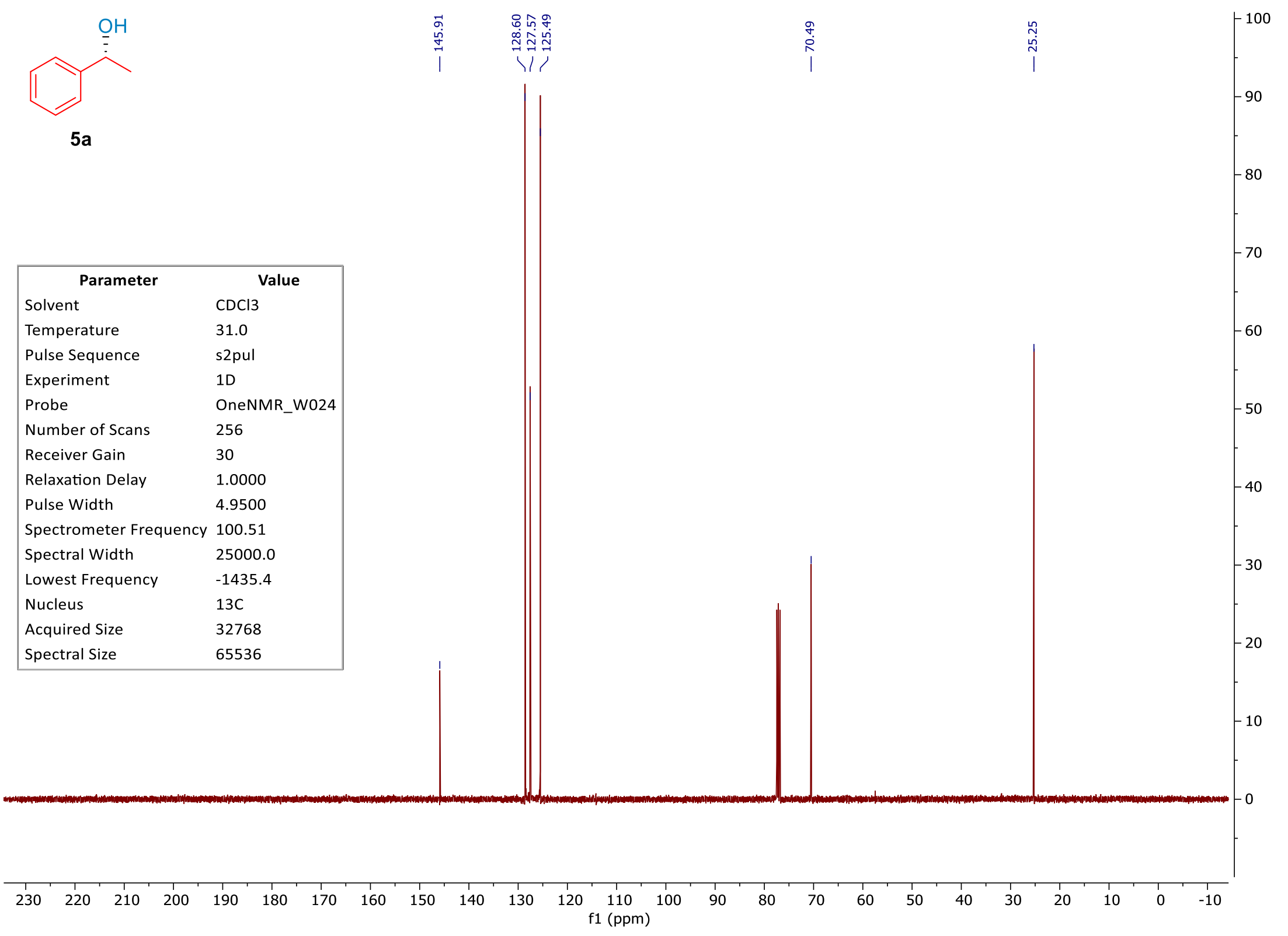

